\title{
Optimal Control of a Variational Inequality with Application to the Kirchhoff Plate Having Small Flexural Rigidity
}

\author{
J. Lovišek
}

\begin{abstract}
This paper concerns an optimal control problem of elliptic singular perturbations in variational inequalities (with controls appearing in coefficients, right-hand sides and convex sets of states as well). The existence of an optimal control is verified. The applications to the optimal design of an elastic plate with a small rigidity and with inner (or moving) obstacle a primal finite element model is applied and convergence result is obtained.
\end{abstract}

Keywords: Optimal control problems, singular perturbations in variational inequalities, convex sets, elastic plates with small rigidity, obstacles

AMS subject classification: 49 A 29, 29 A 27, 29 B 34

\section{Introduction}

The aim of asymptotic methods in optimal control is to simplify the state inequality. The most classical approach is the use of asymptotic expansion in terms of small parameter that may enter the state inequality, i.e. the method of perturbations, in particular the method of singular perturbations. Singular perturbations play a special role as an adequate mathematical tool for describing several important physical phenomena, such as propagation of waves in media in the presence of small energy dissipations or dispersions, appearance of boundary or interior layers in fluid and gas dynamics, as well as in the elasticity theory, semiclassical asymptotic approximations in quantum mechanics, phenomena in the semi-conductor devices theory and so on. We shall deal with singular perturbation of an optimal control problem for an elliptic variational inequality appearing in coefficients, right-hand sides and convex sets of states as well. For the sake of simplicity we confine ourselves to the cases of a linear operator on a Hilbert space. We give first properties of the solutions. Moreover, we shall deal with the discretization of an optimal control problem $(\mathcal{P})$. The existence theorem (for the singular perturbed optimal control) will be applied to the perturbed optimal control of a homogene isotropic plate with small coefficients of the bending rigidity tensor and the membrane (the membrane approximation to the plate obstacle problem is a special example of singular perturbations for elliptic variational inequalities). The numerical

J. Loviśek: Slovak Techn. Univ., Fac. Civil Eng., Radlinskeho 11, 81368 Bratislava, Slovak Republic 
analysis will be restricted to the homogene isotropic plate with small rigidity and with inner obstacle.

Singular perturbations in variational inequalities were considered by Huet [10], Lions [15, 16], Greenlee [8], Eckhaus and Moet [6], Frank [7], and Sanchez-Palencia [22] while those of optimal control problems were considered by Khludnev and Sokolowski [14] and Lions [15]. The main concern is the existence of solution with some weak convergence theorems, but all of the above authors (within Lions, Khludnev and Sokolowski) obtained weak convergence theorems for singular perturbations of variational inequalities.

Before touching the main topic we introduce the notation. Let $H(\Omega)$ be a normed linear space. Following Mosco [19], we introduce the convergence of sequence of subsets:

Definition 1. A sequence $\left\{K_{n}(\Omega)\right\}_{n \in N}$ of subsets of a normal space $H(\Omega)$ converges to a set $K(\Omega) \subset H(\Omega)$ if $K(\Omega)$ contains all weak limits of sequences $\left\{v_{n_{k}}\right\}_{k \in N} \subset K_{n_{k}}(\Omega)$, where $\left\{K_{n_{k}}(\Omega)\right\}_{k \in N}$ are arbitrary subsequences of $\left\{K_{n}(\Omega)\right\}_{n \in N}$ and every element $v \in$ $K(\Omega)$ is the (strong) limit of some sequence $\left\{v_{n}\right\}_{n \in N}, v_{n} \in K_{n}(\Omega)$. We shall write $K(\Omega)=\operatorname{Lim}_{n \rightarrow \infty} K_{n}(\Omega)$ in this situation.

\section{An existence theorem}

Let the control space $U(\Omega)$ be a reflexive Banach space with norm $\|\cdot\|_{U(\Omega)}$, and let $U_{\text {ad }}(\Omega) \subset U(\Omega)$ be a set of admissible controls in $U(\Omega)$. Further, denote by $V(\Omega), W(\Omega)$ two real Hilbert spaces with inner products $(\cdot, \cdot)_{V(\Omega)},(\cdot, \cdot)_{W(\Omega)}$ and norms $\|\cdot\|_{V(\Omega)},\|\cdot\|_{W(\Omega)}$, respectively. Let us denote by $V^{*}(\Omega), W^{*}(\Omega)$ their respective dual spaces of and by $\|\cdot\|_{V *(\Omega)},\left\|_{\cdot}\right\|_{W^{*}(\Omega)}$ their norms with respect to given duality pairings $\langle\cdot, \cdot\rangle_{V(\Omega)},\langle\cdot, \cdot\rangle_{W(\Omega)}$. For a Banach space $\mathcal{H}$ we denote by $L\left(\mathcal{H}, \mathcal{H}^{*}\right)$ the space of all linear continuous operators form $\mathcal{H}$ into $\mathcal{H}^{*}$ endowed with the usual operator norm. For two non-negative constants $\lambda, \Lambda$ we denote by $\mathcal{E}_{\mathcal{H}}(\lambda, \Lambda)$ the set of all symmetric elements $Q$ of $L\left(\mathcal{H}, \mathcal{H}^{*}\right)$ for which the inequalities

$$
\lambda\|v\|_{\mathcal{H}}^{2} \leq\langle Q v, v\rangle_{\mathcal{H}} \quad \text { and } \quad\|Q v\|_{\mathcal{H}^{*}} \leq \Lambda\|v\|_{\mathcal{H}} \quad(v \in \mathcal{H})
$$

hold. We assume that

(No) $V(\Omega) \rightarrow W(\Omega), V(\Omega)$ dense in $W(\Omega)$ and $U_{a d}(\Omega) \subset U(\Omega)$ compact in $U(\Omega)$.

We introduce systems

$$
\{\mathcal{K}(e, \Omega)\}_{e \in U_{a d}(\Omega)} \quad \text { and } \quad\{\mathcal{O}(e, \Omega)\}_{e \in U_{a d}(\Omega)}
$$

of convex closed subsets $\mathcal{K}(e, \Omega) \subset V(\Omega)$ and $\mathcal{O}(e, \Omega) \subset W(\Omega)$, and families of symmetric operators

$$
\{\mathcal{A}(e, \Omega)\}_{e \in U_{a d}(\Omega)} \subset L\left(V(\Omega), V^{*}(\Omega)\right) \text { and }\{\mathcal{B}(e, \Omega)\}_{e \in U_{a d}} \subset L\left(W(\Omega), W^{*}(\Omega)\right)
$$

satisfying the assumptions

$$
\begin{cases}1^{0} & \cap_{e \in U_{a d}(\Omega)} \mathcal{K}(e, \Omega) \neq \emptyset \\ 2^{0} & e_{n} \rightarrow e \text { strongly in } U(\Omega) \Rightarrow \mathcal{K}(e, \Omega)=\operatorname{Lim}_{n \rightarrow \infty} \mathcal{K}\left(e_{n}, \Omega\right) \\ 3^{0} & \{\mathcal{A}(e)\}_{e \in U_{a d}} \subset \mathcal{E}_{V(\Omega)}\left(0, M_{\mathcal{A}}\right) \\ 4^{0} & e_{n} \rightarrow e \text { strongly in } U(\Omega) \Rightarrow \mathcal{A}\left(e_{n}\right) \rightarrow \mathcal{A}(e) \text { in } L\left(V(\Omega), V^{*}(\Omega)\right) \\ 5^{0} & \langle\mathcal{A}(e) v, v\rangle_{V(\Omega)}+\|v\|_{W(\Omega)}^{2} \geq \alpha_{\mathcal{A}}\|v\|_{V(\Omega)}^{2}\left(e \in U_{a d}(\Omega), v \in V(\Omega)\right)\end{cases}
$$


for some $\alpha_{\mathcal{A}}>0$ and

$$
\begin{cases}1^{0} & \mathrm{cl} \mathcal{K}(e, \Omega)=\mathcal{O}(e, \Omega)(\text { closure of } \mathcal{X}(e, \Omega) \text { in } W(\Omega)), e \in U_{a d}(\Omega) \\ 2^{0} & e_{n} \rightarrow e \text { strongly in } U(\Omega) \Rightarrow \mathcal{O}(e, \Omega)=\operatorname{Lim}_{n \rightarrow \infty} \mathcal{O}\left(e_{n}, \Omega\right) \\ 3^{0} & \{\mathcal{B}(e)\}_{e \in U_{\text {ad }}(\Omega)} \subset \mathcal{E}_{W(\Omega)}\left(\alpha_{\mathcal{B}}, M_{\mathcal{B}}\right) \text { with some } \alpha_{\mathcal{B}}>0 \\ 4^{0} & e_{n} \rightarrow e \text { strongly in } U(\Omega) \Rightarrow \mathcal{B}\left(e_{n}\right) \rightarrow \mathcal{B}(e) \text { in } L\left(W(\Omega), W^{*}(\Omega)\right)\end{cases}
$$

Note that $W^{*}(\Omega) \rightarrow V^{*}(\Omega)$ continuously, and one has the transposition formula

$$
\langle F, v\rangle_{V(\Omega)}=\langle F, v\rangle_{W(\Omega)} \quad \forall v \in V(\Omega), F \in W^{*}(\Omega) .
$$

Let $f \in W^{*}(\Omega)$ and $B: U(\Omega) \rightarrow W^{*}(\Omega)$ be a linear continuous operator. For every $\alpha_{B} \geq \varepsilon>0$, and for every $e \in U_{a d}(\Omega)$ there exists a unique state function $u_{\varepsilon}(e) \in \mathcal{K}(e, \Omega)$ such that

$$
\left\langle\varepsilon \mathcal{A}(e) u_{\varepsilon}(e)+\mathcal{B}(e) u_{\varepsilon}(e)-u_{\varepsilon}(e)\right\rangle_{V(\Omega)} \geq\left\langle f+B(e), v-u_{\varepsilon}(e)\right\rangle_{W(\Omega)}
$$

for all $v \in \mathcal{K}(e, \Omega)$. Indeed, thanks to the general theory of variational inequalities [2, $13,21]$ it is enough to prove that there is a constant $c(\varepsilon)>0$ such that

$$
\langle\varepsilon \mathcal{A}(e) v, v\rangle_{V(\Omega)}+\langle\mathcal{B}(e) v, v\rangle_{W(\Omega)} \geq c(\varepsilon)\|v\|_{V(\Omega)}^{2} \quad(v \in V(\Omega)),
$$

and this immediately follows from assumptions $(H 0) / 3^{0}, 5^{0}$ and $(H 1) / 3^{0}$.

Thanks to assumption $(H 1) / 3^{0}$, for any $e \in U_{a d}(\Omega)$ there exists $u(e) \in \mathcal{O}(e, \Omega)$ such that

$$
\langle\mathcal{B}(e) u(e), v-u(e)\rangle_{W(\Omega)} \geq\langle f+B(e), v-u(e)\rangle_{W(\Omega)} \quad(v \in \mathcal{O}(e, \Omega)) .
$$

Let us consider a functional $\mathcal{L}: U(\Omega) \times W(\Omega) \rightarrow \mathbb{R}^{+} \equiv\{a \in \mathbb{R}: a \geq 0\}$ for which the condition

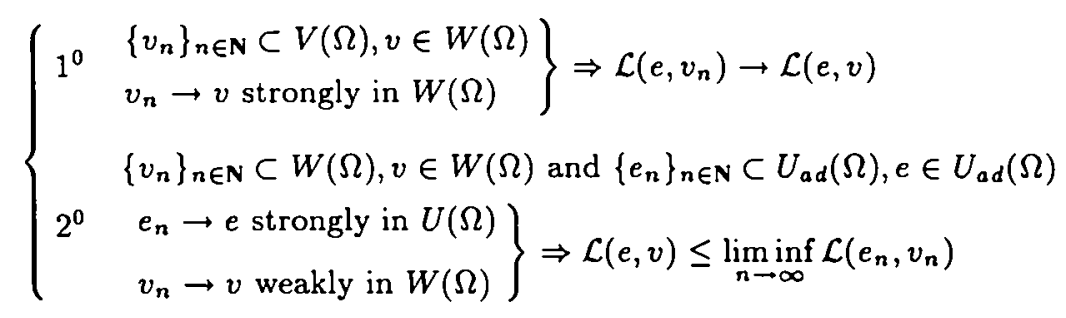

holds. We introduce the functional $J_{e}$ by

$$
J_{\varepsilon}(e)=\mathcal{L}\left(e, u_{\varepsilon}(e)\right) \quad\left(e \in U_{a d}(\Omega)\right)
$$

where $u_{\varepsilon}(e)$ is the uniquely determined solution of (1.3), $e \in U_{a d}(\Omega)$.

We shall solve the following optimisation problem:

$\left(\mathcal{P}_{\varepsilon}\right)$ Find a control $e_{\varepsilon}^{*} \in U_{a d}(\Omega)$ such that $J_{e}\left(e_{\varepsilon}^{*}\right)=\inf _{e \in U_{a d}(\Omega)} J_{e}(e)$.

We say that $e_{\varepsilon}^{*}$ is an optimal control of problem $\left(\mathcal{P}_{e}\right)$. 
Theorem 1. Let assumptions (N0), (H0), (H1) and (E0) be satisfied. Then there exists at least one solution to problem $\left(\mathcal{P}_{\varepsilon}\right)$.

Proof. Due to the compactness of $U_{a d}(\Omega)$ in $U(\Omega)$, there exists a sequence $\left\{e_{\varepsilon}^{n}\right\}_{n \in N}$ $\subset U_{a d}(\Omega)$ such that

$$
\left.\begin{array}{l}
\lim _{n \rightarrow \infty} e_{\varepsilon}^{n}=e_{\varepsilon}^{*} \text { in } U(\Omega) \\
\lim _{n \rightarrow \infty} J_{\varepsilon}\left(e_{\varepsilon}^{n}\right)=\inf _{e \in U_{\text {ad }}(\Omega)} J_{e}(e) .
\end{array}\right\}
$$

Denoting $u_{\varepsilon}\left(e_{\varepsilon}^{n}\right)=: u_{\varepsilon}^{n} \in \mathcal{K}\left(e_{\varepsilon}^{n}, \Omega\right)$ we obtain the inequality

$$
\left\langle\varepsilon \mathcal{A}\left(e_{\varepsilon}^{n}\right) u_{\varepsilon}^{n}+\mathcal{B}\left(e_{\varepsilon}^{n}\right) u_{\varepsilon}^{n}, v-u_{\varepsilon}^{n}\right\rangle_{V(\Omega)} \geq\left\langle f+B e_{\varepsilon}^{n}, v-u_{e}^{n}\right\rangle_{W(\Omega)}
$$

for all $v \in \mathcal{K}\left(e_{\varepsilon}^{n}, \Omega\right)$. Inserting $v=v_{\mathbf{o}} \in \Pi_{e \in U_{\boldsymbol{a}}(\Omega)} \mathcal{X}(e, \Omega)$ into (1.8), we obtain

$$
\begin{aligned}
& \left\langle\varepsilon \mathcal{A}\left(e_{\varepsilon}^{n}\right) u_{\varepsilon}^{n}, u_{\varepsilon}^{n}\right\rangle_{V(\Omega)}+\left\langle\mathcal{B}\left(e_{\varepsilon}^{n}\right) u_{\varepsilon}^{n}, u_{\varepsilon}^{n}\right\rangle_{W(\Omega)} \\
& \quad \leq\left\langle\varepsilon \mathcal{A}\left(e_{\varepsilon}^{n}\right) u_{\varepsilon}^{n}, v_{\mathbf{o}}\right\rangle_{V(\Omega)}+\left\langle\mathcal{B}\left(e_{\varepsilon}^{n}\right) u_{\varepsilon}^{n}, v_{\mathbf{o}}\right\rangle_{W(\Omega)}+\left\langle f+B e_{\varepsilon}^{n}, u_{\varepsilon}^{n}-v_{\mathbf{o}}\right\rangle_{W(\Omega)}
\end{aligned}
$$

for all $n \in \mathbb{N}$. From (1.9) and assumptions $(H 0) / 3^{0}-5^{0}$ and $(H 1) / 3^{0}-4^{0}$ it follows

$$
\left\|u_{\varepsilon}^{n}\right\|_{V(\Omega)} \leq C(\varepsilon) \quad(n \in \mathbb{N}) \quad \text { for fixed } \frac{1}{2} \alpha_{B}>\varepsilon>0 .
$$

It yields the existence of a subsequence $\left\{u_{\varepsilon^{*}}^{n_{k}}\right\}_{k \in N}$ and of an element $u_{\varepsilon}^{*} \in V(\Omega)$ such that

$$
u_{\varepsilon}^{n_{k}} \rightarrow u_{\varepsilon}^{*} \quad \text { weakly in } V(\Omega) .
$$

As $u_{\varepsilon}^{n} \in \mathcal{K}\left(e_{\varepsilon}^{n}, \Omega\right)$, assumption $(H 0) / 2^{0}$ yields

$$
u_{\varepsilon}^{*} \in \mathcal{K}\left(e_{\varepsilon}^{*}, \Omega\right) .
$$

By virtue of assumptions $(H 0) / 3^{0},(H 1) / 3^{0}$ and $(1.10)$ we obtain

$$
\left.\begin{array}{l}
\left\|\mathcal{A}\left(e_{\varepsilon}^{n_{k}}\right) u_{\varepsilon}^{n_{k}}\right\|_{V \cdot(\Omega)} \leq C_{\mathcal{A}}(\varepsilon) \\
\left\|\mathcal{B}\left(e_{\varepsilon}^{n_{k}}\right) u_{e}^{n_{k}}\right\|_{V \cdot(\Omega)} \leq C_{\boldsymbol{B}}(\varepsilon)
\end{array}\right\} \quad(k \in \mathbb{N})
$$

Consequently, there exist subsequences $\left\{\mathcal{A}\left(e_{\varepsilon}^{n_{k_{j}}}\right) u_{\varepsilon}^{n_{k_{j}}}\right\}_{j \in \mathrm{N}},\left\{\mathcal{B}\left(e_{\varepsilon}^{n_{k_{j}}}\right) u_{\varepsilon}^{n_{k_{j}}}\right\}_{j \in \mathrm{N}}$ and elements $\mathcal{X}_{\mathcal{A}} \in V^{*}(\Omega), \mathcal{X}_{B} \in W^{*}(\Omega)$ such that

$$
\left.\begin{array}{l}
\mathcal{A}\left(e_{e}^{n_{k_{j}}}\right) u_{\varepsilon}^{n_{k_{j}}} \rightarrow \mathcal{X}_{\mathcal{A}} \text { weakly in } V^{*}(\Omega) \\
\mathcal{B}\left(e_{e}^{n_{k_{j}}}\right) u_{\varepsilon}^{n_{k_{j}}} \rightarrow \mathcal{X}_{B} \text { weakly in } W^{*}(\Omega) .
\end{array}\right\}
$$

By assumption $(H 0) / 2^{0}$ there exists a sequence $\left\{\Theta_{j}\right\}_{j \in N} \subset \mathcal{K}\left(e_{e}^{n_{k_{j}}}, \Omega\right)$ such that $\Theta_{j} \rightarrow$ $u_{e}^{*}$ in $V(\Omega)$. Henceforth, we often use the implication

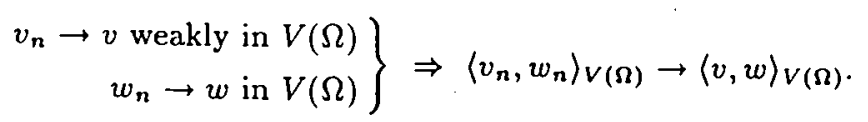


Inserting $v=\Theta_{j}$ into (1.8), we obtain

$$
\begin{aligned}
\limsup _{j \rightarrow \infty} & \left\langle\varepsilon \mathcal{A}\left(e_{\varepsilon}^{n_{k_{j}}}\right) u_{\varepsilon}^{n_{k_{j}}}+\mathcal{B}\left(e_{\varepsilon}^{n_{k_{j}}}\right) u_{\varepsilon}^{n_{k_{j}}}, u_{\varepsilon}^{n_{k_{j}}}\right\rangle_{V(\Omega)} \\
\leq & \limsup _{j \rightarrow \infty}\left\langle\varepsilon \mathcal{A}\left(e_{\varepsilon}^{n_{k_{j}}}\right) u_{\varepsilon}^{n_{k_{j}}}, \Theta_{j}\right\rangle_{V(\Omega)} \\
& +\underset{j \rightarrow \infty}{\limsup }\left\langle\mathcal{B}\left(e_{\varepsilon}^{n_{k_{j}}}\right) u_{\varepsilon}^{n_{k_{j}}}, \Theta_{j}\right\rangle_{W(\Omega)} \\
& +\limsup _{j \rightarrow \infty}\left\langle f+B e_{\varepsilon}^{n_{k_{j}}}, u_{\varepsilon}^{n_{k_{j}}}-\Theta_{j}\right\rangle_{W(\Omega)} \\
= & \left\langle\varepsilon \mathcal{X}_{\mathcal{A}}+\mathcal{X}_{B}, u_{\varepsilon}^{*}\right\rangle_{V(\Omega)}
\end{aligned}
$$

using also (1.14) and the continuity of $B$.

Then due to the monotonicity of $\left[\varepsilon \mathcal{A}\left(e_{e}^{n_{k_{j}}}\right)+\mathcal{B}\left(e_{\varepsilon}^{n_{k_{j}}}\right)\right]$ (by assumptions $(H 0) / 3^{0}$ and $\left.(H 1) / 3^{0}\right)$, we have

$$
\left\langle\left[\varepsilon \mathcal{A}\left(e_{\varepsilon}^{n_{k_{j}}}\right)+\mathcal{B}\left(e_{\varepsilon}^{n_{k_{j}}}\right)\right] u_{\varepsilon}^{n_{k_{j}}}-\left[\varepsilon \mathcal{A}\left(e_{\varepsilon}^{n_{k_{j}}}\right)+\mathcal{B}\left(e_{\varepsilon}^{n_{k_{j}}}\right)\right] v, u_{\varepsilon}^{n_{k_{j}}}-v\right\rangle_{V(\Omega)} \geq 0
$$

for all $v \in V(\Omega)$. From (1.11), (1.14) - (1.15) and assumptions $(H 0) / 4^{0},(H 1) / 4^{0}$ we derive

$$
\left\langle\varepsilon \mathcal{X}_{\mathcal{A}}+\mathcal{X}_{B}-\left[\varepsilon \mathcal{A}\left(e_{\varepsilon}^{*}\right)+\mathcal{B}\left(e_{\varepsilon}^{0}\right)\right] v, u_{\varepsilon}^{*}-v\right\rangle_{V(\Omega)} \geq 0
$$

for all $v \in V(\Omega)$. In fact, on the basic of (1.16) we may write

$$
\begin{aligned}
\limsup _{j \rightarrow \infty} & \left\langle\left[\varepsilon \mathcal{A}\left(e_{\varepsilon}^{n_{k_{j}}}\right)+\mathcal{B}\left(e_{\varepsilon}^{n_{k_{j}}}\right)\right] v, u_{\varepsilon}^{n_{k_{j}}}-v\right\rangle_{V(\Omega)} \\
\leq & \limsup _{j \rightarrow \infty}\left\langle\left[\varepsilon \mathcal{A}\left(e_{\varepsilon}^{n_{k_{j}}}\right)+\mathcal{B}\left(e_{\varepsilon}^{n_{k_{j}}}\right)\right] u_{\varepsilon}^{n_{k_{j}}}, u_{\varepsilon}^{n_{k_{j}}}\right\rangle_{V(\Omega)} \\
& +\limsup _{j \rightarrow \infty}\left\langle\left[\varepsilon \mathcal{A}\left(e_{\varepsilon}^{n_{k_{j}}}\right)+\mathcal{B}\left(e_{\varepsilon}^{n_{k_{j}}}\right)\right] u_{\varepsilon}^{n_{k_{j}}},-v\right\rangle_{V(\Omega)} \\
\leq & \left\langle\varepsilon \mathcal{X}_{\mathcal{A}}+\mathcal{X}_{\mathcal{B}}, u_{\varepsilon}^{*}\right\rangle_{V(\Omega)}+\left\langle\varepsilon \mathcal{X}_{\mathcal{A}}+\mathcal{X}_{B},-v\right\rangle_{V(\Omega)}
\end{aligned}
$$

and (1.17) follows from assumptions $(H 0) / 4^{0},(H 1) / 4^{0}$ and (1.11). Setting

$$
v=u_{e}^{*}+t\left(w-u_{e}^{*}\right) \quad(t \in(0,1), w \in V(\Omega))
$$

we obtain

$$
\left\langle\varepsilon \mathcal{X}_{\mathcal{A}}+\mathcal{X}_{B}-\left[\varepsilon \mathcal{A}\left(e_{\varepsilon}^{*}\right)+\mathcal{B}\left(e_{\varepsilon}^{*}\right)\right]\left(u_{\varepsilon}^{*}+t\left(w-u_{\varepsilon}^{*}\right)\right), u_{\varepsilon}^{*}-v\right\rangle_{V(\Omega)} \geq 0
$$

for all $w \in V(\Omega)$ and $t \in(0,1)$.

Because $\mathcal{A}(e)$ and $\mathcal{B}(e)$ are symmetric and continuous operators we arrive at (inserting again $w=v$ )

$$
\left\langle\left[\varepsilon \mathcal{A}\left(e_{\varepsilon}^{*}\right)+\mathcal{B}\left(e_{\varepsilon}^{*}\right)\right] u_{e}^{*}, u_{e}^{*}-v\right\rangle_{V(\Omega)} \leq\left\langle\varepsilon \mathcal{X}_{\mathcal{A}}+\mathcal{X}_{B}, u_{e}^{*}-v\right\rangle_{V(\Omega)}
$$


for all $v \in V(\Omega)$. Substituting $v=u_{\varepsilon}^{*}$ into (1.16), we obtain

$$
\begin{aligned}
\left\langle\left[\varepsilon \mathcal{A}\left(e_{\varepsilon}^{n_{k_{j}}}\right)\right.\right. & \left.\left.+\mathcal{B}\left(e_{\varepsilon}^{n_{k_{j}}}\right)\right] u_{\varepsilon}^{n_{k_{j}}}, u_{\varepsilon}^{n_{k_{j}}}-u_{\varepsilon}^{*}\right\rangle_{V(\Omega)} \\
\geq & \left\langle\left[\varepsilon \mathcal{A}\left(e_{\varepsilon}^{n_{k_{j}}}\right)+\mathcal{B}\left(e_{\varepsilon}^{n_{k_{j}}}\right)\right] u_{\varepsilon}^{*}, u_{\varepsilon}^{n_{k_{j}}}-u_{\varepsilon}^{*}\right\rangle_{V(\Omega)} .
\end{aligned}
$$

Assumptions $(H 0) / 4^{0},(H 1) / 4^{0}$ and (1.11) imply that

$$
\lim _{j \rightarrow \infty}\left\langle\left[\varepsilon \mathcal{A}\left(e_{\varepsilon}^{n_{k_{j}}}\right)+\mathcal{B}\left(e_{\varepsilon}^{n_{k_{j}}}\right)\right] u_{e}, u_{\varepsilon}^{n_{k_{j}}}-u_{\varepsilon}^{*}\right\rangle_{V(\Omega)}=0
$$

so that

$$
\liminf _{j \rightarrow \infty}\left\langle\left[\varepsilon \mathcal{A}\left(e_{\varepsilon}^{n_{k_{j}}}\right)+\mathcal{B}\left(e_{\varepsilon}^{n_{k_{j}}}\right)\right] u_{\varepsilon}^{n_{k_{j}}}, u_{\varepsilon}^{n_{k_{j}}}-u_{\varepsilon}^{*}\right\rangle_{V(\Omega)} \geq 0 .
$$

Combining this with the inequality

$$
\begin{aligned}
\limsup _{j \rightarrow \infty} & \left\langle\left[\varepsilon \mathcal{A}\left(e_{\varepsilon}^{n_{k_{j}}}\right)+\mathcal{B}\left(e_{\varepsilon}^{n_{k_{j}}}\right)\right] u_{\varepsilon}^{n_{k_{j}}}, u_{\varepsilon}^{n_{k_{j}}}-u_{\varepsilon}^{*}\right\rangle_{V(\Omega)} \\
\leq & \limsup _{j \rightarrow \infty}\left\langle\left[\varepsilon \mathcal{A}\left(e_{\varepsilon}^{n_{k_{j}}}\right)+\mathcal{B}\left(e_{\varepsilon}^{n_{k_{j}}}\right)\right] u_{\varepsilon}^{n_{k_{j}}}, u_{\varepsilon}^{n_{k_{j}}}\right\rangle_{V(\Omega)} \\
& +\lim _{j \rightarrow \infty}\left\langle\left[\varepsilon \mathcal{A}\left(e_{\varepsilon}^{n_{k_{j}}}\right)+\mathcal{B}\left(e_{\varepsilon}^{n_{k_{j}}}\right)\right] u_{\varepsilon}^{n_{k_{j}}},-u_{\varepsilon}^{*}\right\rangle_{V(\Omega)} \\
\leq & 0
\end{aligned}
$$

which is a consequence of (1.15) and (1.14), we are led to the equation

$$
\lim _{j \rightarrow \infty}\left\langle\left[\varepsilon \mathcal{A}\left(e_{\varepsilon}^{n_{k_{j}}}\right)+\mathcal{B}\left(e_{\varepsilon}^{n_{k_{j}}}\right)\right] u_{\varepsilon}^{n_{k_{j}}}, u_{\varepsilon}^{n_{k_{j}}}-u_{\varepsilon}^{*}\right\rangle_{V(\Omega)}=0 .
$$

Given a $v \in \mathcal{K}\left(e_{\varepsilon}^{*}, \Omega\right)$, by assumption $(H 0) / 2^{0}$ there exits a sequence $\left\{v_{j}\right\}_{j \in N} \subset \mathcal{K}\left(e_{\varepsilon}^{n_{k_{j}}}\right.$, $\Omega)$ with $v_{j} \rightarrow v$ strongly in $V(\Omega)$. Inserting $v_{j}$ into (1.8), we have

$$
\begin{aligned}
\lim _{j \rightarrow \infty} & \left\langle\left[\varepsilon \mathcal{A}\left(e_{\varepsilon}^{n_{k_{j}}}\right)+\mathcal{B}\left(e_{\varepsilon}^{n_{k_{j}}}\right)\right] u_{\varepsilon}^{n_{k_{j}}}, u_{\varepsilon}^{n_{k_{j}}}-v_{j}\right\rangle_{V(\Omega)} \\
\leq & \lim _{j \rightarrow \infty}\left\langle f+B e_{\varepsilon}^{n_{k_{j}}}, u_{\varepsilon}^{n_{k_{j}}}-v_{j}\right\rangle_{W(\Omega)} \\
\quad & \left\langle f+B e_{\varepsilon}^{*}, u_{\varepsilon}^{*}-v\right\rangle_{W(\Omega)} .
\end{aligned}
$$

The limit on the left-hand side exists, and furthermore we can write

$$
\begin{aligned}
\lim _{j \rightarrow \infty}\langle & {\left.\left[\varepsilon \mathcal{A}\left(e_{\varepsilon}^{n_{k_{j}}}\right)+\mathcal{B}\left(e_{\varepsilon}^{n_{k_{j}}}\right)\right] u_{\varepsilon}^{n_{k_{j}}}, u_{\varepsilon}^{n_{k_{j}}}-u_{\varepsilon}^{*}\right\rangle_{V(\Omega)} } \\
& +\lim _{j \rightarrow \infty}\left\langle\left[\varepsilon \mathcal{A}\left(e_{\varepsilon}^{n_{k_{j}}}\right)+\mathcal{B}\left(e_{\varepsilon}^{n_{k_{j}}}\right)\right] u_{\varepsilon}^{n_{k_{j}}}, u_{\varepsilon}^{*}-v_{j}\right\rangle_{V(\Omega)} \\
= & \left\langle\varepsilon \mathcal{X}_{\mathcal{A}}+\mathcal{X}_{B}, u_{\varepsilon}^{*}-v_{j}\right\rangle_{V(\Omega)} \\
\geq & \left\langle\left[\varepsilon \mathcal{A}\left(e_{\varepsilon}^{*}\right)+\mathcal{B}\left(e_{\varepsilon}^{*}\right)\right] u_{\varepsilon}^{*}, u_{\varepsilon}^{*}-v\right\rangle_{V(\Omega)}
\end{aligned}
$$

where (1.19), (1.14) and (1.18) have been employed. Consequently,

$$
\left\langle\varepsilon \mathcal{A}\left(e_{\varepsilon}^{*}\right) u_{\varepsilon}^{*}, v-u_{\varepsilon}^{*}\right\rangle_{V(\Omega)}+\left\langle\mathcal{B}\left(e_{\varepsilon}^{*}\right) u_{\varepsilon}^{*}, v-u_{\varepsilon}^{*}\right\rangle_{W(\Omega)} \geq\left\langle f+B e_{\varepsilon}^{*}, v-u_{e}^{*}\right\rangle_{W(\Omega)},
$$

and as $v \in \mathcal{K}\left(e_{\varepsilon}^{*}, \Omega\right)$ is chosen arbitrary, we get

$$
u_{\varepsilon}^{*} \equiv u_{\varepsilon}\left(e_{\varepsilon}^{*}\right) \quad \text { and } \quad u_{\varepsilon}\left(e_{\varepsilon}^{n_{k}}\right) \rightarrow u_{\varepsilon}\left(e_{\varepsilon}^{*}\right) \text { weakly in } V(\Omega) .
$$

Then assumption $(E 0) / 2^{0}$ and (1.21) yield

$$
\mathcal{L}\left(e_{\varepsilon}^{* 0}, u_{e}\left(e_{\varepsilon}^{*}\right)\right)=\liminf _{k \rightarrow \infty} \mathcal{L}\left(e_{e}^{n_{k}}, u_{\varepsilon}\left(e_{e}^{n_{k}}\right)\right)=\inf _{e \in U_{\text {ad }}(\Omega)} \mathcal{L}\left(e, u_{\varepsilon}(e)\right) .
$$

Hence $\mathcal{L}\left(e_{\varepsilon}^{*}, u_{e}\left(e_{\varepsilon}^{*}\right)\right)=\inf \left\{\mathcal{L}\left(e, u_{\varepsilon}(e)\right): e \in U_{\text {ad }}(\Omega)\right\}$ which completes the proof 
Limit state function and limit cost function. We define the limit state function $u_{0}(e)$ for any $e \in U_{a d}(\Omega)$ by the following variational inequality: Find $u_{0}(e) \in \mathcal{O}(e, \Omega)$ such that

$$
\left\langle\mathcal{B}(e) u_{0}(e), v-u_{0}(e)\right\rangle_{W(\Omega)} \geq\left\langle f+B e, v-u_{0}(e)\right\rangle_{W(\Omega)} \quad(v \in \mathcal{O}(e, \Omega))
$$

Further, we define the limit cost function $J_{0}(e)$ by

$$
J_{0}(e)=\mathcal{L}\left(e, u_{0}(e)\right)
$$

In this case one has the following limit control problem:

$\left(\mathcal{P}_{0}\right)$ Find $e_{0}^{*}$ such that $J_{0}\left(e_{0}^{*}\right)=\inf _{e \in U_{\text {ad }}(\Omega)} J_{0}(e)$.

Theorem 2. Let assumptions $(N 0),(H 1)$ and $(E 0)$ be satisfied. Then there exists at least one solution to problem $\left(\mathcal{P}_{0}\right)$.

Proof. The proof is analogous to that of Theorem 1 and hence it is omitted

There arises a natural question concerning the type of relation between solution to problems $\left(\mathcal{P}_{0}\right)$ and $\left(\mathcal{P}_{\varepsilon}\right)$ as $\varepsilon \rightarrow 0_{+}$. We prove the following theorem.

Theorem 3. Let assumptions $(N 0),(H 0),(H 1)$ and $(E 0)$ be satisfied. Let $e_{\varepsilon_{n}}^{*}$ be the solution to problem $\left(\mathcal{P}_{\varepsilon_{n}}\right)$ and $\varepsilon_{n} \rightarrow 0_{+}$. Then there exists a subsequence $\left\{\varepsilon_{n_{k}}\right\}_{k \in \mathbb{N}}$ of $\left\{\varepsilon_{n}\right\}_{n \in N}$ and a solution $e_{0}^{*}$ of problem $\left(\mathcal{P}_{0}\right)$ such that

$$
\left.\begin{array}{r}
e_{e_{n_{k}}^{*}} \rightarrow e_{0}^{*} \text { strongly in } U(\Omega) \\
J_{e_{n_{k}}}\left(e_{\varepsilon_{n_{k}}^{*}}^{*}\right)=\inf _{e \in U_{a d}(\Omega)} J_{\varepsilon_{n_{n_{k}}}}\left(e_{e_{n_{k}}^{*}}\right) \rightarrow u_{0}\left(e_{0}^{*}\right) \text { weakly in } W(\Omega) \\
\rightarrow J_{0}\left(e_{0}^{*}\right)=\inf _{e \in U_{a d}(\Omega)} J_{0}(e) .
\end{array}\right\}
$$

Proof. Due to the compactness of $U_{a d}(\Omega)$ there exists $e_{0} \in U_{a d}(\Omega)$ AND a subsequence of $\left\{e_{e_{n}}^{*}\right\}_{n \in N} \subset U_{a d}(\Omega)$ denoted again by $\left\{e_{\varepsilon_{n}}^{*}\right\}_{n \in N}$ such that $e_{\varepsilon_{n}}^{*} \rightarrow e_{0}$ strongly in $U(\Omega)$. Then the state function $u_{e_{n}}\left(e_{\varepsilon_{n}}^{*}\right) \in \mathcal{K}\left(e_{e_{n}}^{*}, \Omega\right)$ is a solution of the state variational inequality

$$
\begin{array}{r}
\left\langle\varepsilon_{n} \mathcal{A}\left(e_{\varepsilon_{n}}^{*}\right) u_{e_{n}}\left(e_{e_{n}}^{*}\right)+\mathcal{B}\left(e_{\varepsilon_{n}}^{*}\right) u_{e_{n}}\left(e_{e_{n}}^{*}\right), v-u_{e_{n}}\left(e_{e_{n}}^{*}\right)\right\rangle_{V(\Omega)} \\
\geq\left\langle f+B e_{e_{n}}^{*}, v-u_{e_{n}}\left(e_{\varepsilon_{n}}^{*}\right)\right\rangle_{W(\Omega)}
\end{array}
$$

for any $v \in \mathcal{K}\left(e_{\varepsilon_{n}}^{*}, \Omega\right)$ and for given $e_{\varepsilon_{n}}^{*} \in U_{a d}(\Omega)$ with $\varepsilon_{n}>0$ ( $\left.n \in \mathbb{N}\right)$. Taking $v=v_{0} \in \cap_{e \in U_{a d}(\Omega)} \mathcal{K}(e, \Omega)$ fixed in inequality (1.26) we obtain

$$
\begin{aligned}
\left\langle\varepsilon_{n} \mathcal{A}\left(e_{\varepsilon_{n}}^{*}\right) u_{\varepsilon_{n}}\left(e_{e_{n}}^{*}\right)+\mathcal{B}\left(e_{\varepsilon_{n}}^{*}\right) u_{\varepsilon_{n}}\left(e_{\varepsilon_{n}}^{*}\right), u_{\varepsilon_{n}}\left(e_{e_{n}}^{*}\right)\right\rangle_{V(\Omega)} \\
\left.\leq \leq f+B e_{\varepsilon_{n}}^{*}, u_{\varepsilon_{n}}\left(e_{\varepsilon_{n}}^{*}\right)-v_{0}\right\rangle_{W(\Omega)} \\
\quad+\left\langle\varepsilon_{n} \mathcal{A}\left(e_{\varepsilon_{n}}^{*}\right) u_{\varepsilon_{n}}\left(e_{\varepsilon_{n}}^{*}\right)+\mathcal{B}\left(e_{e_{n}}^{*}\right) u_{\varepsilon_{n}}\left(e_{\varepsilon_{n}}^{*}\right), v_{0}\right\rangle_{V(\Omega)}
\end{aligned}
$$


It follows that

$$
\begin{aligned}
\varepsilon_{n} & \left(\left\langle\mathcal{A}\left(e_{\varepsilon_{n}}^{*}\right) u_{\varepsilon_{n}}\left(e_{\varepsilon_{n}}^{*}\right), u_{\varepsilon_{n}}\left(e_{\varepsilon_{n}}^{*}\right)\right\rangle_{V(\Omega)}\right. \\
& \left.+\left\|u_{\varepsilon_{n}}\left(e_{\varepsilon_{n}}^{*}\right)\right\|_{W(\Omega)}^{2}\right)+\left(\alpha_{\mathcal{B}}-\varepsilon_{n}\right)\left\|u_{\varepsilon_{n}}\left(e_{\varepsilon_{n}}^{*}\right)\right\|_{W(\Omega)}^{2} \\
\leq & \left\langle f+B e_{\varepsilon_{n}}^{*}, u_{e_{n}}\left(e_{\varepsilon_{n}}^{*}\right)-v_{*}\right\rangle_{W(\Omega)} \\
& +\left\langle\varepsilon_{n} \mathcal{A}\left(e_{\varepsilon_{n}}^{*}\right) u_{\varepsilon_{n}}\left(e_{\varepsilon_{n}}^{*}\right), v_{*}\right\rangle_{V(\Omega)}+\left\langle\mathcal{B}\left(e_{\varepsilon_{n}}^{*}\right) u_{\varepsilon_{n}}\left(e_{\varepsilon_{n}}^{*}\right), v_{*}\right\rangle_{W(\Omega)}
\end{aligned}
$$

Then we obtain by setting $\frac{1}{2} \alpha_{B} \geq \varepsilon_{n}>0$ and applying assumptions $(H 0)$ and $(H 1)$

$$
\begin{aligned}
\left(\varepsilon_{n} \alpha_{\mathcal{A}}\right)\left\|u_{\varepsilon_{n}}\left(e_{\varepsilon_{n}}^{*}\right)\right\|_{V(\Omega)}^{2}+\frac{1}{2} \alpha_{\mathcal{B}}\left\|u_{\varepsilon_{n}}\left(e_{\varepsilon_{n}}^{*}\right)\right\|_{W(\Omega)}^{2} \\
\leq c_{1}\left\|u_{\varepsilon_{n}}\left(e_{\varepsilon_{n}}^{*}\right)-v_{0}\right\|_{W(\Omega)} \\
+c_{2} \varepsilon_{n}\left\|u_{\varepsilon_{n}}\left(e_{\varepsilon_{n}}^{*}\right)\right\| V(\Omega)\left\|v_{\mathrm{o}}\right\|_{V(\Omega)} \\
+c_{3}\left\|u_{\varepsilon_{n}}\left(e_{\varepsilon_{n}}^{*}\right)\right\| W(\Omega)\left\|v_{0}\right\|_{W(\Omega)}
\end{aligned}
$$

where given constants $c_{1}, c_{2}, c_{3}$ do not depend on $\varepsilon$. From it we conclude that

$$
\left.\begin{array}{r}
\left\|u_{\varepsilon_{n}}\left(e_{\varepsilon_{n}}^{*}\right)\right\|_{W(\Omega)} \leq c \\
\sqrt{\varepsilon_{n}}\left\|u_{\varepsilon_{n}}\left(e_{e_{n}}^{*}\right)\right\|_{V(\Omega)} \leq c .
\end{array}\right\}
$$

Wc can therefore extract a subsequence $\left\{u_{\varepsilon_{n_{k}}}\left(e_{e_{n_{k}}^{*}}\right)\right\}_{k \in N}$ such that

$$
\left.\begin{array}{rl}
u_{\varepsilon_{n_{k}}}\left(e_{\varepsilon_{n_{k}}^{*}}^{*}\right) & \rightarrow w \text { weakly in } W(\Omega) \\
\sqrt{\varepsilon_{n_{k}}} u_{\varepsilon_{n_{k}}}\left(e_{\varepsilon_{n_{k}}}^{*}\right) & \rightarrow 0 \text { weakly in } V(\Omega)
\end{array}\right\} \quad(k \rightarrow \infty)
$$

Since $u_{\varepsilon_{n_{k}}}\left(e_{\varepsilon_{n_{k}}}^{*}\right) \in \mathcal{K}\left(e_{\varepsilon_{n_{k}}^{*}}^{*}, \Omega\right)$ by assumption $(H 0) / 2^{0}$, we have $w \in \mathcal{K}\left(e_{0}, \Omega\right)$ as well. From this one has $w \in \mathcal{O}\left(e_{0}, \Omega\right)$. For any $z \in V(\Omega)$ we have by assumption $(H 0) / 4^{0}$ and by virtue of (1.28)

$$
\begin{aligned}
\lim _{k \rightarrow \infty}\left\langle\mathcal{A}\left(e_{\varepsilon_{n_{k}}^{*}}^{*}\right) \sqrt{\varepsilon_{n_{k}}} u_{\varepsilon_{n_{k}}}\left(e_{\varepsilon_{n_{k}}^{*}}^{*}\right), z\right\rangle_{V(\Omega)} & =\lim _{k \rightarrow \infty}\left\langle\mathcal{A}\left(e_{\varepsilon_{n_{k}}}^{*}\right) z, \sqrt{\varepsilon_{n_{k}}} u_{\varepsilon_{n_{k}}}\left(e_{\varepsilon_{n_{k}}}^{*}\right)\right\rangle_{V(\Omega)} \\
& =\left\langle\mathcal{A}\left(e_{0}\right) z, 0\right\rangle_{V(\Omega)} \\
& =\left\langle\mathcal{A}\left(e_{0}\right) 0, z\right\rangle_{V(\Omega)}
\end{aligned}
$$

and therefore

$$
\mathcal{A}\left(e_{\varepsilon_{n_{k}}}^{*}\right) \sqrt{\varepsilon_{n_{k}}} u_{\varepsilon_{n_{k}}}\left(e_{\varepsilon_{n_{k}}^{*}}^{*}\right) \rightarrow \mathcal{A}\left(e_{0}\right) 0 \equiv 0 \quad \text { weakly in } V^{*}(\Omega)
$$

as $k \rightarrow \infty$ (note that $\left|\left\langle\varepsilon \mathcal{A}(e) v, u_{\varepsilon}\right\rangle_{V(\Omega)}\right|=O(\sqrt{\varepsilon})$ ). On the other hand, by analogy of (1.29) we obtain

$$
\begin{aligned}
\lim _{k \rightarrow \infty}\left\langle\mathcal{B}\left(e_{\varepsilon_{n_{k}}}^{*}\right) u_{\varepsilon_{n_{k}}}\left(e_{\varepsilon_{n_{k}}^{*}}^{*}\right), z\right\rangle_{W(\Omega)} & =\lim _{k \rightarrow \infty}\left\langle\mathcal{B}\left(e_{\varepsilon_{n_{k}}^{*}}^{*}\right) z, u_{\varepsilon_{n_{k}}}\left(e_{e_{n_{k}}}^{*}\right)\right\rangle_{W(\Omega)} \\
& =\left\langle\mathcal{B}\left(e_{0}\right) z, w\right\rangle_{W(\Omega)}
\end{aligned}
$$


This means that

$$
\mathcal{B}\left(e_{\varepsilon_{n_{k}}}^{*}\right) u_{e_{n_{k}}}\left(e_{\varepsilon_{n_{k}}^{*}}^{*}\right) \rightarrow \mathcal{B}\left(e_{0}\right) w \quad \text { weakly in } W^{*}(\Omega)
$$

as $k \rightarrow \infty$. Furthermore, in virtue of the monotonicity of $\mathcal{B}\left(e_{e_{n_{k}}^{*}}\right)$ (due to assumption $(H 1) / 3^{0}$ ) we know that

$$
\left\langle\mathcal{B}\left(e_{\varepsilon_{n_{k}}}^{*}\right) u_{\varepsilon_{n_{k}}}\left(e_{\varepsilon_{n_{k}}}^{*}\right), u_{e_{n_{k}}}\left(e_{\varepsilon_{n_{k}}^{*}}^{*}\right)-w\right\rangle_{W(\Omega)} \geq\left\langle\mathcal{B}\left(e_{\varepsilon_{n_{k}}}^{*}\right) w, u_{\varepsilon_{n_{k}}}\left(e_{\varepsilon_{n_{k}}^{*}}^{*}\right)-w\right\rangle_{W(\Omega)}
$$

for all $k \in \mathbb{N}$. Passing to the limit we get

$$
\begin{aligned}
& 2 \lim _{k \rightarrow \infty}\left\langle\mathcal{B}\left(e_{\varepsilon_{n_{k}}}^{*}\right) w, u_{\varepsilon_{n_{k}}}\left(e_{\varepsilon_{n_{k}}^{*}}^{*}\right)\right\rangle_{W(\Omega)} \\
& \quad \leq \liminf _{k \rightarrow \infty}\left\langle\mathcal{B}\left(e_{\varepsilon_{n_{k}}^{*}}^{*}\right) u_{\varepsilon_{n_{k}}}\left(e_{\varepsilon_{n_{k}}^{*}}^{*}\right), u_{\varepsilon_{n_{k}}}\left(e_{\varepsilon_{n_{k}}}^{*}\right)\right\rangle_{W(\Omega)}+\lim _{k \rightarrow \infty}\left\langle\mathcal{B}\left(e_{\varepsilon_{n_{k}}}^{*}\right) w, w\right\rangle_{W(\Omega)} .
\end{aligned}
$$

This yields, together with (1.28), assumption $(H 1) / 4^{0}$ and (1.30),

$$
\liminf _{k \rightarrow \infty}\left\langle\mathcal{B}\left(e_{\varepsilon_{n_{k}}}^{*}\right) u_{\varepsilon_{n_{k}}}\left(e_{e_{n_{k}}^{*}}^{*}\right), u_{\varepsilon_{n_{k}}}\left(e_{\varepsilon_{n_{k}}}^{*}\right)\right\rangle_{W(\Omega)} \geq\left\langle\mathcal{B}\left(e_{0}\right) w, w\right\rangle_{W(\Omega)}
$$

Let $v \in \mathcal{K}\left(e_{0}, \Omega\right)$ be an arbitrary element and $\left\{v_{k}\right\}_{k \in N}$ such a sequence that

$$
\left.v_{k} \in \mathcal{K}\left(e_{\varepsilon_{n_{k}}^{*}}^{*}, \Omega\right), \text { for given } e_{\varepsilon_{n_{k}}^{*}} \in \begin{array}{r}
v_{k} \rightarrow v \text { strongly in } \dot{V}(\Omega) \\
U_{a d}(\Omega), \varepsilon_{n_{k}}>0(k \in \mathbb{N})
\end{array}\right\}
$$

(the existence of such a sequence is ensured by assumption $(H 0) / 2^{0}$ ). Then we have

$$
\begin{array}{r}
\left\langle\varepsilon_{n_{k}} \mathcal{A}\left(e_{\varepsilon_{n_{k}}}^{*}\right) u_{\varepsilon_{n_{k}}}\left(e_{\varepsilon_{n_{k}}^{*}}^{*}\right)+\mathcal{B}\left(e_{\varepsilon_{n_{k}}^{*}}^{*}\right) u_{\varepsilon_{n_{k}}}\left(e_{\varepsilon_{n_{k}}^{*}}\right), v_{k}\right\rangle_{V(\Omega)} \\
-\left\langle f+B e_{\varepsilon_{n_{k}}^{*}}, v_{k}-u_{\varepsilon_{n_{k}}}\left(e_{\varepsilon_{n_{k}}^{*}}^{*}\right)\right\rangle_{W(\Omega)} \\
\geq\left\langle\mathcal{B}\left(e_{\varepsilon_{n_{k}}^{*}}\right) u_{\varepsilon_{n_{k}}}\left(e_{\varepsilon_{n_{k}}^{*}}^{*}\right), u_{\varepsilon_{n_{k}}}\left(e_{\varepsilon_{n_{k}}^{*}}^{*}\right)\right\rangle_{W(\Omega)} .
\end{array}
$$

From this inequality using (1.28), (1.31) and (1.32) we get

$$
\left\langle\mathcal{B}\left(e_{0}\right) w, v-w\right\rangle_{W(\Omega)}-\left\langle f+B e_{0}, v-w\right\rangle_{W(\Omega)} \geq 0 \quad\left(v \in \mathcal{K}\left(e_{0}, \Omega\right)\right)
$$

and therefore we have also (1.33) for all $v \in \mathcal{O}\left(e_{0}, \Omega\right)$ (by density). This yields $w=$ $u_{0}\left(e_{0}\right)$ since the variational inequality (1.23) has a unique solution for $e \in U_{a d}(\Omega)$. Consequently, it may be supposed that

$$
\left.\begin{array}{rl}
e_{\varepsilon_{n_{k}}}^{*} & \rightarrow e_{0} \text { strongly in } U(\Omega) \\
u_{e_{n_{k}}}\left(e_{e_{n_{k}}}^{*}\right) & \rightarrow u_{0}\left(e_{0}\right) \text { weakly in } W(\Omega)
\end{array}\right\}
$$

for $k \rightarrow \infty\left(\varepsilon_{n_{k}} \rightarrow 0\right)$. 
Now, let us consider regarding to (1.26) and (1.2)

$$
\begin{aligned}
\left\langle\varepsilon_{n} \mathcal{A}\left(e_{\varepsilon_{n}}^{*}\right) u_{\varepsilon_{n}}\left(e_{\varepsilon_{n}}^{*}\right)+\mathcal{B}\left(e_{\varepsilon_{n}}^{*}\right) u_{\varepsilon_{n}}\left(e_{\varepsilon_{n}}^{*}\right), u_{e_{n}}\left(e_{\varepsilon_{n}}^{*}\right)\right\rangle_{V(\Omega)} \\
\leq\left\langle\varepsilon_{n} \mathcal{A}\left(e_{\varepsilon_{n}}^{*}\right) u_{\varepsilon_{n}}\left(e_{\varepsilon_{n}}^{*}\right)+\mathcal{B}\left(e_{\varepsilon_{n}}^{*}\right) u_{\varepsilon_{n}}\left(e_{\varepsilon_{n}}^{*}\right), v_{n}\right\rangle_{V(\Omega)} \\
-\left\langle f+B e_{\varepsilon_{n}}^{*}, v_{n}-u_{\varepsilon_{n}}\left(e_{\varepsilon_{n}}^{*}\right)\right\rangle_{W(\Omega)}
\end{aligned}
$$

where $u_{\varepsilon_{n}}\left(e_{e_{n}}^{*}\right), v_{n} \in \mathcal{K}\left(e_{\varepsilon_{n}}^{*}, \Omega\right)$ and $e_{\varepsilon_{n}}^{*} \in U_{a d}(\Omega)$. We deduce from (1.35) that

$$
\begin{aligned}
& \limsup _{k \rightarrow \infty}\left\langle\mathcal{B}\left(e_{\varepsilon_{n}}^{*}\right) u_{\varepsilon_{n}}\left(e_{\varepsilon_{n}}^{*}\right), u_{\varepsilon_{n}}\left(e_{\varepsilon_{n}}^{*}\right)\right\rangle_{W(\Omega)} \\
& \quad \leq \lim _{k \rightarrow \infty}\left(\left\langle\mathcal{B}\left(e_{\varepsilon_{n}}^{*}\right) u_{\varepsilon_{n}}\left(e_{\varepsilon_{n}}^{*}\right), v_{n}\right\rangle_{W(\Omega)}-\left\langle f+B e_{\varepsilon_{n}}^{*}, v_{n}-u_{\varepsilon_{n}}\left(e_{\varepsilon_{n}}^{*}\right)\right\rangle_{W(\Omega)}\right) .
\end{aligned}
$$

Hence by (1.32), (1.30), (1.34) and the continuity of $B$ one has

$$
\begin{aligned}
& \lim _{k \rightarrow \infty} \sup \left\langle\mathcal{B}\left(e_{\varepsilon_{n}}^{*}\right) u_{\varepsilon_{n}}\left(e_{\varepsilon_{n}}^{*}\right), u_{e_{n}}\left(e_{\varepsilon_{n}}^{*}\right)\right\rangle_{W(\Omega)} \\
& \quad \leq\left\langle\mathcal{B}\left(e_{0}\right) u_{0}\left(e_{0}\right), v\right\rangle_{W(\Omega)}-\left\langle f+B e_{0}, v-u_{0}\left(e_{0}\right)\right\rangle_{W(\Omega)}
\end{aligned}
$$

for all $v \in \mathcal{K}\left(e_{0}, \Omega\right)$ (by density one concludes (1.37) also for all $v \in \mathcal{O}\left(e_{0}, \Omega\right)$ ) and therefore (by taking $v=u_{0}\left(e_{0}\right) \in \mathcal{O}\left(c_{0}, \Omega\right)$ in (1.37)) the inequality

$$
\lim _{n \rightarrow \infty} \sup \left\langle\mathcal{B}\left(e_{e_{n}}^{*}\right) u_{e_{n}}\left(e_{\varepsilon_{n}}^{*}\right), u_{\varepsilon_{n}}\left(e_{\varepsilon_{n}}^{*}\right)\right\rangle_{W(\Omega)} \leq\left\langle\mathcal{B}\left(e_{0}\right) u_{0}\left(e_{0}\right), u_{0}\left(e_{0}\right)\right\rangle_{W(\Omega)}
$$

is verified. Using it we get via (1.31), (1.34) and (1.38)

$$
\lim _{n \rightarrow \infty}\left\langle\mathcal{B}\left(e_{\varepsilon_{n}}^{*}\right) u_{e_{n}}\left(e_{\varepsilon_{n}}^{*}\right), u_{e_{n}}\left(e_{e_{n}}^{*}\right)\right\rangle_{W(\Omega)}=\left\langle\mathcal{B}\left(e_{0}\right) u_{0}\left(e_{0}\right), u_{0}\left(e_{0}\right)\right\rangle_{W(\Omega)} .
$$

Moreover, the method of the proof shows that for $e \in U_{a d}(\Omega)$ the convergence

$$
u_{\varepsilon_{n}}(e) \rightarrow u_{0}(e) \quad \text { strongly in } W(\Omega) \text { when } \varepsilon_{n} \rightarrow 0_{+}
$$

holds.

One has

$$
\begin{aligned}
\mathcal{N}_{n}= & \left\langle\mathcal{B}(e)\left(u_{\varepsilon_{n}}(e)-u_{0}(e)\right), u_{\varepsilon_{n}}(e)-u_{0}(e)\right\rangle_{W(\Omega)} \\
= & \left\langle\mathcal{B}(e) u_{\varepsilon_{n}}(e)-u_{0}(e)\right\rangle_{W(\Omega)}-\left\langle\mathcal{B}(e) u_{0}(e), u_{\varepsilon_{n}}(e)-u_{0}(e)\right\rangle_{W(\Omega)} \\
\leq & \left\langle\mathcal{B}(e) u_{\varepsilon_{n}}(e), u_{\varepsilon_{n}}(e)\right\rangle_{W(\Omega)} \\
& -\left\langle\mathcal{B}(e) u_{\varepsilon_{n}}(e), u_{0}(e)\right\rangle_{W(\Omega)}-\left\langle f+B e, u_{\varepsilon_{n}}(e)-u_{0}(e)\right\rangle_{W(\Omega)}
\end{aligned}
$$

with $u_{e_{n}}(e) \in \mathcal{K}(e, \Omega)$ and $u_{0}(e) \in \mathcal{O}(e, \Omega)$. But

$$
\begin{aligned}
&\left\langle\mathcal{B}(e) u_{e_{n}}(e), u_{e_{n}}(e)\right\rangle_{W(\Omega)} \\
& \leq\left\langle\varepsilon_{n} \mathcal{A}(e) u_{\varepsilon_{n}}(e)+\mathcal{B}(e) u_{e_{n}}(e), u_{e_{n}}(e)\right\rangle_{V(\Omega)} \\
& \leq\left\langle\varepsilon_{n} \mathcal{A}(e) u_{\varepsilon_{n}}(e), v\right\rangle_{V(\Omega)} \\
&+\left\langle\varepsilon_{n} \mathcal{A}(e) u_{\varepsilon_{n}}(e)+\mathcal{B}(e) u_{\varepsilon_{n}}(e)-v\right\rangle_{V(\Omega)}+\left\langle\mathcal{B}(e) u_{e_{n}}(e), v\right\rangle_{W(\Omega)} \\
& \leq\left\langle\varepsilon_{n} \mathcal{A}(e) u_{e_{n}}(e), v\right\rangle_{V(\Omega)} \\
&+\left\langle\mathcal{B}(e) u_{\varepsilon_{n}}(e), v\right\rangle_{W(\Omega)}+\left\langle f+B e, u_{\varepsilon_{n}}(e)-v\right\rangle_{W(\Omega)}
\end{aligned}
$$


for $v$ fixed in $\mathcal{K}(e, \Omega) \subset \mathcal{O}(e, \Omega)$. From (1.41) and (1.42) one can find

$$
\begin{aligned}
\mathcal{N}_{n} \leq & \left\langle f+B e, u_{0}(e)-v\right\rangle_{W(\Omega)} \\
& +\left\langle\mathcal{B}(e) u_{e_{n}}(e), v-u_{0}(e)\right\rangle_{W(\Omega)}+\left\langle\varepsilon_{n} \mathcal{A}(e) u_{e_{n}}(e), v\right\rangle_{V(\Omega)}
\end{aligned}
$$

On the other hand, we may write

$$
\begin{aligned}
& \left\langle\varepsilon_{n} \mathcal{A}(e) u_{\varepsilon_{n}}(e)+\mathcal{B}(e) u_{\varepsilon_{n}}(e), u_{\varepsilon_{n}}(e)\right\rangle_{V(\Omega)} \\
& \quad \leq\left\langle f+B e, u_{\varepsilon_{n}}(e)-v\right\rangle_{W(\Omega)}+\left\langle\varepsilon_{n} \mathcal{A}(e) u_{\varepsilon_{n}}(e), v\right\rangle_{V(\Omega)}+\left\langle\mathcal{B}(e) u_{\varepsilon_{n}}(e), v\right\rangle_{W(\Omega)}
\end{aligned}
$$

and this yields due to assumption $(H 0) / 5^{\circ}$

$$
\begin{aligned}
& \varepsilon_{n} \alpha_{\mathcal{A}}\left\|u_{e_{n}}(e)\right\|_{V(\Omega)}^{2}+\frac{\alpha_{\mathcal{B}}}{2}\left\|u_{\varepsilon_{n}}(e)\right\|_{W(\Omega)}^{2} \\
& \quad \leq c_{a}\left\|u_{e_{n}}(e)-v\right\| w(\Omega)+\varepsilon_{n} c_{b}\left\|u_{\varepsilon_{n}}(e)\right\| V(\Omega)+c_{c}\left\|u_{\varepsilon_{n}}(e)\right\|_{W(\Omega)}
\end{aligned}
$$

where $c_{a}, c_{b}, c_{c}$ are some constants with respect to $n$.

Thus one can finds $\left\|u_{\varepsilon_{n}}(e)\right\|_{W(\Omega)} \leq c$ and $\sqrt{\varepsilon_{n}}\left\|u_{e_{n}}(e)\right\|_{v(\Omega)} \leq c$. So there exists $u_{\varepsilon_{n_{k}}}(e) \rightarrow w$ weakly in $W(\Omega)$ and $\sqrt{\varepsilon_{n_{k}}} u_{\varepsilon_{n_{k}}}(e) \rightarrow 0$ weakly in $V(\Omega)$ for $k \rightarrow \infty\left(\varepsilon_{n_{k}} \rightarrow\right.$ $0)$. Supposing $n_{k}=n$, we have

$$
\begin{aligned}
\lim _{n \rightarrow \infty}\left\langle\mathcal{A}(e) \sqrt{\varepsilon_{n}} u_{\varepsilon_{n}}(e), z\right\rangle_{V(\Omega)} & =\lim _{n \rightarrow \infty}\left\langle\mathcal{A}(e) z, \sqrt{\varepsilon_{n}} u_{\varepsilon_{n}}(e)\right\rangle_{V(\Omega)} \\
& =\langle\mathcal{A}(e) z, 0\rangle_{V(\Omega)} \\
& =0 \\
& =\langle\mathcal{A}(e) 0, z\rangle_{V(\Omega)} .
\end{aligned}
$$

Thus one has

$$
\mathcal{A}(e) \sqrt{\varepsilon_{n}} u_{\varepsilon_{n}}(e) \rightarrow \mathcal{A}(e) 0=0 \quad \text { weakly in } V^{*}(\Omega)
$$

We have also

$$
\lim _{n \rightarrow \infty}\left\langle\mathcal{B}(e) u_{e_{n}}(e), z\right\rangle_{W(\Omega)}=\langle\mathcal{B}(e) w, z\rangle_{W(\Omega)}
$$

which means

$$
\mathcal{B}(e) u_{c_{n}}(e) \rightarrow \mathcal{B}(e) w \quad \text { weakly in } W^{*}(\Omega)
$$

Now by (1.43) - (1.45) we see that

$$
\lim _{n \rightarrow \infty} \mathcal{N}_{n} \leq\left\langle f+B e, u_{0}(e)-v\right\rangle_{W(\Omega)}+\left\langle\mathcal{B}(e) w, v-u_{0}(e)\right\rangle_{W(\Omega)}+0
$$

for each $v \in \mathcal{K}(e, \Omega)$. By density this inequality holds true for $v \in \mathcal{O}(e, \Omega)$ and by replacing $v=u_{0}(e)$ one finds $\lim \sup \mathcal{N}_{n}=0$. Thus one has

$$
\limsup _{n \rightarrow \infty} \alpha_{B}\left\|u_{e_{n}}(e)-u_{0}(e)\right\|_{W(\Omega)}^{2}=0
$$

which means that $u_{\varepsilon_{n}}(e) \rightarrow u_{0}(e)$ strongly in $W(\Omega)$. 
From (1.40), from the fact that $J_{\varepsilon_{n}}\left(e_{\varepsilon_{n}}^{*}\right) \leq J_{\varepsilon_{n}}(e)$ for all $e \in U_{a d}(\Omega)$ and from assumption $(E 0) / 1^{0}$ we get

$$
\left.\begin{array}{r}
\limsup _{k \rightarrow \infty} J_{\varepsilon_{n}}\left(e_{\epsilon_{n}}^{*}\right) \leq J_{0}(e) \\
\quad \text { for all } e \in U_{a d}(\Omega)
\end{array}\right\} \Rightarrow \limsup _{k \rightarrow \infty} J_{\varepsilon_{n}}\left(e_{\varepsilon_{n}}^{*}\right) \leq \inf _{e \in U_{a d}(\Omega)} J_{0}(e)=J_{0}\left(e_{0}^{*}\right) .
$$

Furthermore, we observe that assumption $(E 0) / 2^{0}$ and (1.34) imply

$$
\liminf _{k \rightarrow \infty} J_{\varepsilon_{n_{k}}}\left(e_{\varepsilon_{n_{k}}}^{*}\right) \geq \mathcal{L}\left(e_{0}, u_{0}\left(e_{0}\right)\right)=J_{0}\left(e_{0}\right) .
$$

Comparing this result with (1.47) we have $J_{0}\left(e_{0}\right) \leq J_{0}\left(e_{0}^{*}\right)$. Thus we see that necessarily $e_{0}=e_{0}^{*}$ and $(1.47)$ and $(1.48)$ give $(1.25)_{3}$. Theorem 3 is proved

\section{Approximation of the optimal control problem by discretization}

Let us assume that $U_{a d}(\Omega) \subset U(\Omega)$ is compact. We describe the discretization of problem $\left(\mathcal{P}_{\varepsilon}\right)$ and we prove the convergence of the sequence of finite-dimensional solutions as $h$, the discretization parameter, tends to zero. Let $\mathcal{K}(e, \Omega)$ and $\mathcal{O}(e, \Omega)$ be two closed convex sets in the spaces $V(\Omega)$ and $W(\Omega)$, respectively, for all $e \in U_{a d}(\Omega)$. With any $h \in(0,1)$ we associate

$$
\begin{cases}1^{0} & \text { finite-dimensional subspaces } V_{h}(\Omega) \subset V(\Omega) \text { and } U^{h}(\Omega) \subset U(\Omega) \\ 2^{0} & \text { closed convex subsets } \left.\mathcal{K}_{h}\left(e_{h}, \Omega\right) \subset V_{h}(\Omega) \text { (approximations of } \mathcal{K}(e, \Omega)\right) \\ 3^{0} & \text { closed convex subsets } \left.U_{a d}^{h}(\Omega) \subset U^{h}(\Omega) \text { (approximations of } U_{a d}(\Omega)\right) \\ 4^{0} & \text { bilinear forms } a_{h}\left(e_{h}, \cdot\right)\left(\equiv \left\langle\mathcal{A}_{h}\left(e_{h}\right) \cdot, \cdot V_{h}(\Omega): V_{h}(\Omega) \times V_{h}(\Omega) \rightarrow \mathbb{R}\right.\right. \\ & e_{h} \in U_{a d}^{h}(\Omega), \text { together with the operators } \mathcal{A}_{h}\left(e_{h}\right): V_{h}(\Omega) \rightarrow V_{h}^{*}(\Omega) \\ & (\text { approximation of } a(e, \cdot, \cdot)) \\ 5^{0} & \mathcal{L}_{h}: U^{h}(\Omega) \times V_{h}(\Omega) \rightarrow \mathbb{R} \text { convex lower semicontinuous proper functionals } \\ & (\text { approximations of the cost functional } \mathcal{L}) .\end{cases}
$$

Analogously, with any $h \in(0,1)$ we associate

$$
\begin{cases}1^{0} & \text { finite-dimensional subspaces } W_{h}(\Omega) \subset W(\Omega) \text { and } V_{h}(\Omega) \subset W_{h}(\Omega) \\ 2^{0} & \text { closed convex subsets } \left.\mathcal{O}_{h}\left(e_{h}, \Omega\right) \subset W_{h}(\Omega) \text { (approximations of } \mathcal{O}(e, \Omega)\right) \\ 3^{0} & \text { bilinear forms } b_{h}\left(e_{h}, \cdot, \cdot\right)\left(\equiv\left\langle\mathcal{B}_{h}\left(e_{h}\right), \cdot\right\rangle_{W_{h}(\Omega)}\right): W_{h}(\Omega) \times W_{h}(\Omega) \rightarrow \mathbb{R}, \\ & \left.e_{h} \in U_{a d}^{h}(\Omega), \mathcal{B}_{h}\left(e_{h}\right): W_{h}(\Omega) \rightarrow W_{h}^{*}(\Omega) \text { (approximation of } b(e, \cdot, \cdot)\right) \\ 4^{0} & \left.f_{h} \in W_{h}^{*}(\Omega), B_{h} \in L\left(U^{h}(\Omega), W_{h}^{*}(\Omega)\right) \text { (approximations of } f \text { and } B\right) .\end{cases}
$$

The families $\left\{\mathcal{K}_{h_{n}}\left(e_{h_{n}}, \Omega\right)\right\}_{n \in N}$ and $\left\{\mathcal{O}_{h_{n}}\left(e_{h_{n}}, \Omega\right)\right\}_{n \in \mathbb{N}}$ are supposed to satisfy the condition

$$
\begin{aligned}
& \left(1^{0} \quad h_{n} \rightarrow 0_{+}, e_{h_{n}} \rightarrow e \text { strongly in } U^{h_{n}}(\Omega) \text { such that } e_{h_{n}} \in U_{\text {ad }}^{h_{n}}(\Omega)\right. \\
& \text { for any } n \in \mathbb{N} \Rightarrow \text { for any bounded sequence }\left\{v_{h_{n}}\right\}_{n \in \mathbf{N}} \text { such that } \\
& (\text { Lo })_{\mathcal{A}_{\mathrm{b}}} \quad \begin{cases}v_{h_{n}} \in \mathcal{K}_{h_{n}}\left(e_{h_{n}}, \Omega\right) \text { all its weak cluster points belong to } \mathcal{K}(e, \Omega) & \text { There are } \Lambda_{\mathcal{K}(e, \Omega)} \subset V(\Omega), \operatorname{cl} \Lambda_{\mathcal{K}(e, \Omega)}=\mathcal{K}(e, \Omega), \text { such that }\end{cases} \\
& \text { for any } h_{n} \rightarrow 0_{+} \text {and } e_{h_{n}} \rightarrow e \text { strongly in } U^{h_{n}}(\Omega) \text { there is } \\
& \mathcal{R}_{e_{h_{n}} e}: \Lambda_{\mathcal{K}(e, \Omega)} \rightarrow \mathcal{K}_{h_{n}}\left(e_{h_{n}}, \Omega\right) \text { such that for all } v \in \Lambda_{\mathcal{K}(e, \Omega)} \\
& \text { we have } \lim _{n \rightarrow 0} \mathcal{R}_{e_{h_{n}}} e v=v \text { strongly in } V_{h_{n}}(\Omega)
\end{aligned}
$$


or the condition

$(\mathrm{LO})_{\boldsymbol{B}_{\mathrm{h}}} \quad \begin{cases} & \text { all its weak cluster points belong to } \mathcal{O}(e, \Omega) \\ 2^{0} & \text { There are } \Lambda_{\mathrm{o}(e, \Omega)} \subset W(\Omega), \operatorname{cl} \Lambda_{\mathrm{o}(e, \Omega)}=\mathcal{O}(e, \Omega) \text { such that }\end{cases}$ for any $h_{n} \rightarrow 0_{+}$and $e_{h_{n}} \rightarrow e$ strongly in $U^{h_{n}}(\Omega)$ there is $\mathcal{V}_{e_{h_{n}} e}: \Lambda_{o(e, \Omega)} \rightarrow \mathcal{O}_{h_{n}}\left(e_{h_{n}}, \Omega\right)$ such that for all $v \in \Lambda_{o(e, \Omega)}$ we have $\lim _{n \rightarrow 0} \mathcal{V}_{e_{h_{n}}}$ e $v_{e}=v$ strongly in $W_{h_{n}}(\Omega)$.

Let us note that we do not necessarily have $\mathcal{K}_{h}\left(e_{h}, \Omega\right) \subset \mathcal{K}(e, \Omega), \mathcal{O}_{h}\left(e_{h}, \Omega\right) \subset$ $\mathcal{O}(e, \Omega)$ or $U_{a d}^{h}(\Omega) \subset U_{a d}(\Omega)$. If, however, this is true for any $h \in(0,1)$, we say that we have an internal approximation of $\mathcal{K}(e, \Omega), \mathcal{O}(e, \Omega)$ or $U_{a d}(\Omega)$, respectively.

The approximation of the state inequality (1.3) is now defined by means of the RitzGalerkin procedure. This method will perform well if $\varepsilon \geq h$, but if $\varepsilon \ll h$, then this method may produce an oscillating solution which is not close to the exact solution (see an example 9.1 in [12] or in [24]). However, if the exact solution happens to be smooth, then the standard Ritz-Galerkin method will produce good results even if $\varepsilon<h$. The approximation of (1.3) reads as follows:

Find

$$
u_{\varepsilon h}\left(e_{h}\right) \in \mathcal{K}_{h}\left(e_{h}, \Omega\right)
$$

such that

$$
\begin{gathered}
\left\langle\varepsilon \mathcal{A}_{h}\left(e_{h}\right) u_{\varepsilon h}\left(e_{h}\right)+\mathcal{B}_{h}\left(e_{h}\right) u_{e h}\left(e_{h}\right), v_{h}-u_{c h}\left(e_{h}\right)\right\rangle_{V_{h}(\Omega)} \\
\geq\left\langle f_{h}+B_{h} e_{h}, v_{h}-u_{\varepsilon h}\left(e_{h}\right)\right\rangle_{W_{h}(\Omega)}
\end{gathered}
$$

for any $v_{h} \in \mathcal{K}_{h}\left(e_{h}, \Omega\right)$ and $e_{h} \in U_{a d}^{h}(\Omega)$ and

$$
\left.\begin{array}{r}
u_{0 h}\left(e_{h}\right) \in \mathcal{O}_{h}\left(e_{h}, \Omega\right) \\
\left\langle\mathcal{B}_{h}\left(e_{h}\right) u_{0 h}\left(e_{h}\right), v_{h}-u_{0 h}\left(e_{h}\right)\right\rangle_{W_{h}(\Omega)} \geq\left\langle f_{h}+B e_{h}, v_{h}-u_{0 h}\left(e_{h}\right)\right\rangle_{W_{h}(\Omega)} \\
\text { for any } v_{h} \in \mathcal{O}_{h}\left(e_{h}, \Omega\right) \text { and } e_{h} \in U_{a d}^{h}(\Omega) .
\end{array}\right\}
$$

For a set $M$ and a function $\mathcal{H}: M \rightarrow \mathbb{R}$ we denote by $\operatorname{Argmin}_{M} \mathcal{H}$ the set of minimizers of $\mathcal{H}$ on $M$. Thus, the discrete versions of problems $\left(\mathcal{P}_{\varepsilon}\right)$ and $\left(\mathcal{P}_{0}\right)$ read as

$$
\left(\mathcal{P}_{\varepsilon h}\right) \text { Find } e_{\varepsilon h}^{*} \in \underset{e_{h} \in U_{a d}^{h}(\Omega)}{\operatorname{Argmin}} \mathcal{L}_{h}\left(e_{h}, u_{e h}\left(e_{h}\right)\right) \equiv \underset{e_{h} \in U_{a d}^{h}(\Omega)}{\operatorname{Argmin}} J_{e h}\left(e_{h}\right)
$$

with $u_{\varepsilon h}\left(e_{h}\right)$ as above and

$$
\left(\mathcal{P}_{0 h}\right) \text { Find } e_{0 h}^{*} \in \underset{e_{h} \subset U_{a d}^{h}(\Omega)}{\operatorname{Argmin}} \mathcal{L}_{h}\left(e_{h}, u_{0 h}\left(e_{h}\right)\right) \equiv \underset{e_{h} \in U_{a d}^{h}(\Omega)}{\operatorname{Argmin}} J_{0 h}\left(e_{h}\right)
$$

with $u_{0 h}\left(e_{h}\right)$ as above, and the control problems $\left(\mathcal{P}_{e}\right)$ and $\left(\mathcal{P}_{0}\right)$ reads as

$$
\left(\mathcal{P}_{\varepsilon}\right) \text { Find } e_{\varepsilon}^{*} \in \underset{e \in U_{\text {ad }}(\Omega)}{\operatorname{Arginf}} \mathcal{L}\left(e, u_{\varepsilon}(e)\right) \equiv \underset{e \in U_{\text {ad }}(\Omega)}{\operatorname{Arginf}} J_{\varepsilon}(e) \text {. }
$$

with $u_{e}(e)$ as above and 
$\left(\mathcal{P}_{0}\right)$ Find $e_{0}^{*} \in \underset{e \in U_{a d}(\Omega)}{\operatorname{Arginf}} \mathcal{L}\left(e, u_{0}(e)\right) \equiv \underset{e \in U_{a d}(\Omega)}{\operatorname{Arginf}} J_{0}(e)$

with $u_{0}(e)$ as above.

In what follows, we shall study the relation between optimal pairs of problems $\left(\mathcal{P}_{c h}\right)$ and $\left(\mathcal{P}_{\varepsilon}\right)$ as $h \rightarrow 0_{+}$, for any fixed $\varepsilon>h$.

For the analysis of the relation between (1.3), (2.1) and the relation between (1.5), (2.2) we shall need the hypotheses concerning $\mathcal{A}_{h_{n}}\left(e_{h_{n}}\right)$ :

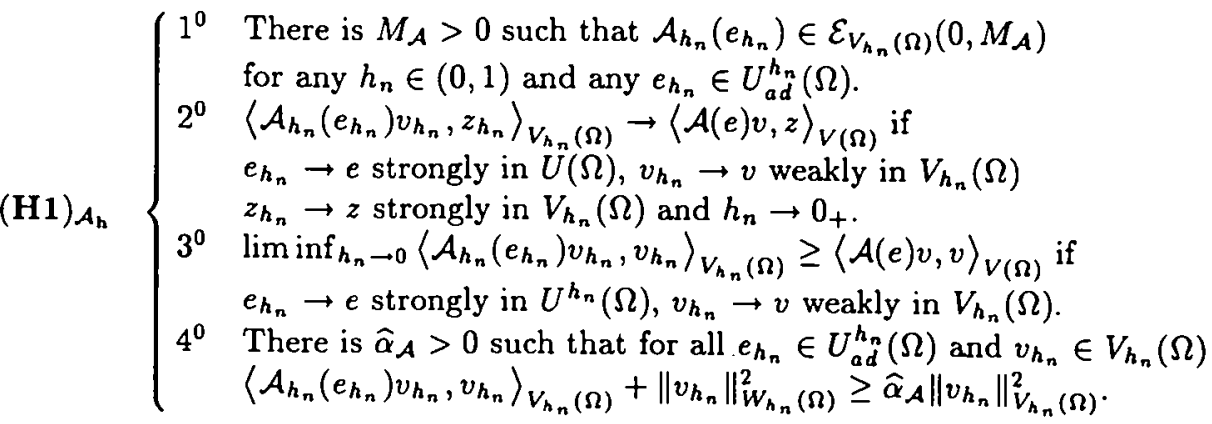

Moreover, we suppose the following hypotheses concerning $\mathcal{B}_{h_{n}}\left(e_{h_{n}}\right)$ :

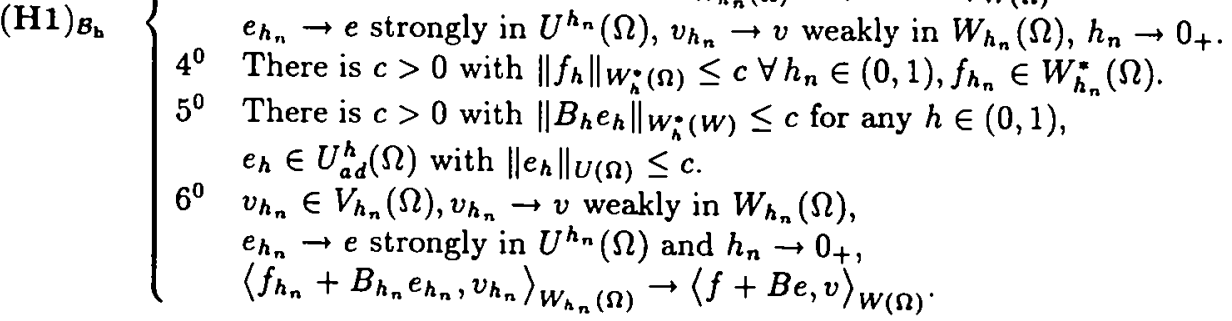

Next we assume that

$(\mathrm{E} 0)_{\mathrm{h}} \begin{cases}1^{0} & v_{h}^{n} \in V_{h}(\Omega) \text { and } v_{h}^{n} \rightarrow v_{h} \text { strongly in } V_{h}(\Omega) \\ & \Rightarrow \mathcal{L}_{h}\left(e_{h}, v_{h}\right)=\lim _{n \rightarrow \infty} \mathcal{L}_{h}\left(e_{h}, v_{h}^{n}\right) . \\ 2^{0} & e_{h}^{n} \in U_{a d}^{h}(\Omega), e_{h}^{n} \rightarrow e_{h} \text { strongly in } U^{h}(\Omega), \\ & v_{h}^{n} \in V_{h}(\Omega), v_{h}^{n} \rightarrow v_{h} \text { strongly in } V_{h}(\Omega) \\ & \Rightarrow \mathcal{L}_{h}\left(e_{h}, v_{h}\right) \leq \liminf \operatorname{in}_{n \rightarrow \infty} \mathcal{L}_{h}\left(e_{h}^{n}, v_{h}^{n}\right) .\end{cases}$

For every $h>0, \hat{\alpha}_{B} \geq \varepsilon>h, e_{h} \in U_{a d}^{h}(\Omega)$ there exists a unique solution $u_{e h}\left(e_{h}\right) \in$ $\mathcal{K}_{h}\left(e_{h}, \Omega\right)$ of the variational inequality

$$
\begin{gathered}
\left\langle\varepsilon \mathcal{A}_{h}\left(e_{h}\right) u_{\varepsilon h}\left(e_{h}\right)+\mathcal{B}_{h}\left(e_{h}\right) u_{\varepsilon h}\left(e_{h}\right), v_{h}-u_{\varepsilon h}\left(e_{h}\right)\right\rangle_{V_{h}(\Omega)} \\
\geq\left\langle f_{h}+B_{h} e_{h}, v_{h}-u_{\varepsilon h}\left(e_{h}\right)\right\rangle_{W_{h}(\Omega)}
\end{gathered}
$$


for all $v_{h} \in \mathcal{K}_{h}\left(e_{h}, \Omega\right)$. Indeed, due to assumptions $(H 1)_{\mathcal{A}_{h}} / 4^{0}$ and $(H 1)_{B_{h}} / 1^{0}$ there exits a constant $c_{\mathcal{A B}}(\varepsilon)>0$ such that

$$
\left\langle\varepsilon \mathcal{A}_{h}\left(e_{h}\right) v_{h}, v_{h}\right\rangle_{V_{h}(\Omega)}+\left\langle\mathcal{B}_{h}\left(e_{h}\right) v_{h}, v_{h}\right\rangle_{W_{h}(\Omega)} \geq c_{\mathcal{A B}}(\varepsilon)\left\|v_{h}\right\|_{V_{h}(\Omega)}^{2}
$$

for any $v_{h} \in V_{h}(\Omega), e_{h} \in U_{a d}^{h}(\Omega)$ and for any $\varepsilon$ with $\widehat{\alpha}_{B} \geq \varepsilon>h$.

Lemma 1. For every $h>0$ and for every $\varepsilon$ with $\frac{1}{2} \widehat{\alpha}_{B} \geq \varepsilon>h$ there exists at least one optimal pair $\left[e_{e h}^{*}, u_{e h}\left(e_{e h}^{*}\right)\right]$ for problem $\left(\mathcal{P}_{\varepsilon h}\right)$.

Proof. It is quite analogous to that of Theorem 1 and hence it is omitted

Lemma 2. Under the above hypotheses $(L 0)_{\mathcal{A}_{h}},(H 1)_{\mathcal{A}_{h}}$ and $(H 1)_{B_{h}}$, let $e_{h_{n}} \in$ $U_{a d}^{h}(\Omega)$ be such that $e_{h_{n}} \rightarrow$ e strongly in $U^{h_{n}}(\Omega)$ as $h_{n} \rightarrow 0_{+}$. Then $u_{e h_{n}}\left(e_{e h_{n}}\right) \rightarrow u_{\varepsilon}(e)$ strongly in $V_{h_{n}}(\Omega)$, for any fixed $\varepsilon$ with $\frac{1}{2} \widehat{\alpha}_{B} \geq \varepsilon>h_{n}$.

Proof. We take an arbitrary o $\in \mathcal{K}(e, \Omega)$ and by assumption $(L 0)_{\mathcal{A}_{h}} / 2^{0}$ a sequence $\left\{\mathcal{R}_{e_{h_{n}} e} \mathbf{o}\right\}_{n \in N} \in \Pi_{n \in N} \mathcal{K}_{h_{n}}\left(e_{h_{n}}, \Omega\right)$ such that $\mathcal{R}_{e_{h_{n}} \text { e }} \rightarrow$ o. Putting $v_{h_{n}}=\mathcal{R}_{e_{h_{n}} \text { e }}$ in (2.1), adding

$$
\begin{aligned}
& \left\langle\varepsilon \mathcal{A}_{h_{n}}\left(e_{h_{n}}\right) \mathcal{R}_{e_{h_{n}} e} \mathbf{o}, u_{\epsilon_{h_{n}}}\left(e_{h_{n}}\right)-\mathcal{R}_{e_{h_{n}} e} \mathbf{0}\right\rangle_{V_{h_{n}}(\Omega)} \\
& \quad+\left\langle\mathcal{B}_{h_{n}}\left(e_{h_{n}}\right) \mathcal{R}_{e_{h_{n}} e} \mathbf{0}, u_{e_{h_{n}}}\left(e_{h_{n}}\right)-\mathcal{R}_{e_{h_{n}} e} \mathbf{0}\right\rangle_{W_{h_{n}}(\Omega)}
\end{aligned}
$$

to its both sides, and multiplying the resulting inequality by minus one, we obtain

$$
\begin{aligned}
& \left\langle\varepsilon\left(\mathcal{A}_{h_{n}}\left(e_{h_{n}}\right) u_{\varepsilon h_{n}}\left(e_{h_{n}}\right)-\mathcal{A}_{h_{n}}\left(e_{h_{n}}\right) \mathcal{R}_{e_{h_{n}} e} 0\right), u_{\varepsilon h_{n}}\left(e_{h_{n}}\right)-\mathcal{R}_{e_{h_{n}} e} \mathbf{o}\right\rangle_{V_{h_{n}}(\Omega)} \\
& +\left\langle\mathcal{B}_{h_{n}}\left(e_{h_{n}}\right) u_{e h_{n}}\left(e_{h_{n}}\right)-\mathcal{B}_{h_{n}}\left(e_{h_{n}}\right) \mathcal{R}_{e_{h_{n}} e} 0, u_{e h_{n}}\left(e_{h_{n}}\right)-\mathcal{R}_{e_{h_{n}} e} \mathbf{o}\right\rangle_{W_{h_{n}}(\Omega)} \\
& \leq\left\langle\varepsilon \mathcal{A}_{h_{n}}\left(e_{h_{n}}\right) \mathcal{R}_{e_{h_{n}} e} \mathbf{O}, \mathcal{R}_{e_{h_{n}} e} \mathbf{O}-u_{\varepsilon h_{n}}\left(e_{h_{n}}\right)\right\rangle_{V_{h_{n}}(\Omega)} \\
& +\left\langle\mathcal{B}_{h_{n}}\left(e_{h_{n}}\right) \mathcal{R}_{e_{h_{n}} e} \mathcal{O}, \mathcal{R}_{e_{h_{n}} e} \mathrm{O}-u_{e h_{n}}\left(e_{h_{n}}\right)\right\rangle_{W_{h_{n}}(\Omega)} \\
& +\left\langle f_{h_{n}}+B_{h_{n}} e_{h_{n}}, u_{\varepsilon h_{n}}\left(e_{h_{n}}\right)-\mathcal{R}_{e_{h_{n}} e} \mathbf{o}\right\rangle_{W_{h_{n}}(\Omega)}
\end{aligned}
$$

for all $n \in \mathbb{N}$. Then due to assumptions $(H 1)_{\mathcal{A}_{h}},(H 1)_{\mathcal{B}_{h}}$ and (2.5) we arrive at the estimate

$$
\left\|u_{e h_{n}}\left(e_{h_{n}}\right)\right\|_{V_{h_{n}}(\Omega)} \leq c(\varepsilon) \quad(n \in \mathbb{N})
$$

valid for $\varepsilon>0$, with positive $c(\varepsilon)$ independent of $n \in \mathbb{N}$. Thus there exists a subsequence $\left\{u_{\varepsilon h_{n_{k}}}\left(e_{h_{n_{k}}}\right)\right\}_{k \in N}$ of $\left\{u_{\varepsilon h_{n}}\left(e_{h_{n}}\right)\right\}_{n \in N}$ and an element $u_{\varepsilon} \in V(\Omega)$ such that $u_{\varepsilon h_{n_{k}}}\left(e_{h_{n_{k}}}\right) \rightarrow$ $u_{\varepsilon}$ weakly in $V_{h_{n_{k}}}(\Omega)$ for $k \rightarrow \infty$, for any fixed $\varepsilon>0$. Moreover, we have $u_{\varepsilon} \in$ $\mathcal{K}(e, \Omega)$ due to assumption $(L 0)_{\mathcal{A}_{h}} / 1^{0}$. On the other hand, by virtue of assumption $(L 0)_{\mathcal{A}_{h}} / 2^{0}$, for soine $a \in \Lambda_{\mathcal{K}(e, \Omega)}$ we obtain the existence of a sequence $\left\{\mathcal{R}_{e_{n_{n_{k}}} a} a\right\}_{k \in N} \in$ $\Pi_{k \in N} \mathcal{K}_{h_{n_{k}}}\left(e_{h_{n_{k}}}, \Omega\right)$ such that $\lim _{k \rightarrow \infty} \mathcal{R}_{e_{n_{n_{k}}} e} a=a$ strongly in $V_{h_{n_{k}}}(\Omega)$ as $e_{h_{n_{k}}} \rightarrow e$ strongly in $U^{h_{n_{k}}}(\Omega)$. Taking into account and inserting $v_{h}:=\mathcal{R}_{e_{n_{n_{k}}}} a$ into (2.1), adding

$$
\left\langle\varepsilon \mathcal{A}_{h_{n_{k}}}\left(e_{h_{n_{k}}}\right) \mathcal{R}_{e_{h_{n_{k}}}} a+\mathcal{B}_{h_{n_{k}}}\left(e_{h_{n_{k}}}\right) \mathcal{R}_{e_{n_{n_{k}}} e} a, u_{e_{n_{n_{k}}}}\left(e_{h_{n_{k}}}\right)-\mathcal{R}_{e_{n_{n_{k}}} e} a\right\rangle_{V_{h_{n_{k}}}(\Omega)}
$$


to its both sides, and multiplying the resulting by minus one, we obtain

$$
\begin{aligned}
& \limsup _{k \rightarrow \infty}\left(\left(\varepsilon \mathcal{A}_{h_{n_{k}}}\left(e_{h_{n_{k}}}\right)+\mathcal{B}_{h_{n_{k}}}\left(e_{h_{n_{k}}}\right)\right)\left(u_{\varepsilon_{h_{n_{k}}}}\left(e_{h_{n_{k}}}\right)-\mathcal{R}_{e_{h_{n_{k}}}} a\right),\right. \\
& \left.u_{e_{n_{n_{k}}}}\left(e_{h_{n_{k}}}\right)-\mathcal{R}_{e_{h_{n_{k}}}} a\right\rangle_{V_{h_{n_{k}}}(\Omega)} \\
& \leq \underset{k \rightarrow \infty}{\limsup }\left|\left\langle\varepsilon \mathcal{A}_{h_{n_{k}}}\left(e_{h_{n_{k}}}\right) \mathcal{R}_{e_{h_{n_{k}}}} a, \mathcal{R}_{e_{n_{n_{k}}} e} a-u_{e_{n_{n_{k}}}}\left(e_{h_{n_{k}}}\right)\right\rangle_{V_{h_{n_{k}}}(\Omega)}\right| \\
& +\underset{k \rightarrow \infty}{\limsup }\left|\left\langle\mathcal{B}_{h_{n_{k}}}\left(e_{h_{n_{k}}}\right) \mathcal{R}_{e_{h_{n_{k}}}} a, \mathcal{R}_{e_{h_{n_{k}}}} a-u_{e_{n_{n_{k}}}}\left(e_{h_{n_{k}}}\right)\right\rangle_{W_{h_{n_{k}}}(\Omega)}\right| \\
& +\underset{k \rightarrow \infty}{\limsup }\left|\left\langle f_{h_{n_{k}}}+B_{h_{n_{k}}} e_{h_{n_{k}}}, u_{e_{n_{n_{k}}}}\left(e_{h_{n_{k}}}\right)-\mathcal{R}_{e_{h_{n_{k}}} a} a\right\rangle_{W_{h_{n_{k}}}(\Omega)}\right| \\
& =0 \text {. }
\end{aligned}
$$

The last equality follows from (1.2) and from the facts

$$
\left.\begin{array}{r}
e_{h_{n}} \rightarrow e \text { strongly in } U^{h_{n}}(\Omega) \text { and } v_{h_{n}} \rightarrow v \text { strongly in } V_{h_{n}}(\Omega) \\
\Rightarrow\left\|\mathcal{A}_{h_{n}}\left(e_{h_{n}}\right) v_{h_{n}}-\mathcal{A}(e) v\right\|_{v_{h_{n}}(\Omega)} \leq M_{\mathcal{A}}\left\|v_{h_{n}}-v\right\| v_{h_{n}}(\Omega) \\
+\left\|\mathcal{A}_{h_{n}}\left(e_{h_{n}}\right) v-\mathcal{A}(e) v\right\|_{v_{h_{n}}(\Omega)} \text { for } n \rightarrow \infty
\end{array}\right\}
$$

and

$$
\begin{aligned}
& \left.e_{h_{n}} \rightarrow e \text { strongly in } U^{h_{n}}(\Omega) \text { and } z_{h_{n}} \rightarrow z \text { strongly in } W_{h_{n}}(\Omega)\right) \\
& \Rightarrow\left\|\mathcal{B}_{h_{n}}\left(e_{h_{n}}\right) z_{h_{n}}-\mathcal{B}(e) z\right\|_{W_{h_{n}}(\Omega)} \leq M_{\mathcal{B}}\left\|z_{h_{n}}-z\right\|_{W_{h_{n}}(\Omega)} \\
& +\left\|\mathcal{B}_{h_{n}}\left(e_{h_{n}}\right) z-\mathcal{B}(e) z\right\|_{W_{h_{n}}(\Omega)} \rightarrow 0 \text { for } n \rightarrow \infty
\end{aligned}
$$

which are consequences of assumptions $(H 1)_{\mathcal{A}_{h}} / 1^{0}, 2^{0}$ and $(H 1)_{B_{h}} / 1^{0}, 2^{0}$, respectively. So by the uniform monotonicity of $\left[\varepsilon \mathcal{A}_{h_{n_{k}}}\left(e_{h_{n_{k}}}\right)+\mathcal{B}_{h_{n_{k}}}\left(e_{h_{n_{k}}}\right)\right]$ we obtain the convergence

$$
u_{\varepsilon h_{n k}}\left(e_{h_{n_{k}}}\right) \rightarrow u_{\varepsilon} \quad \text { strongly in } V_{h_{n_{k}}}(\Omega)
$$

for $k \rightarrow \infty$. Moreover, (2.9) together with (2.7) and (2.8) yields

$$
\left.\begin{array}{l}
\mathcal{A}_{h_{n_{k}}}\left(e_{h_{n_{k}}}\right) u_{e h_{n_{k}}}\left(e_{h_{n_{k}}}\right) \rightarrow \mathcal{A}(e) u_{\varepsilon} \text { strongly in } V_{h_{n_{k}}}^{*}(\Omega) \\
\mathcal{B}_{h_{n_{k}}}\left(e_{h_{n_{k}}}\right) u_{e h_{n_{k}}}\left(e_{h_{n_{k}}}\right) \rightarrow \mathcal{B}(e) u_{\varepsilon} \text { strongly in } W_{h_{n_{k}}}^{*}(\Omega)
\end{array}\right\} \quad(k \rightarrow \infty)
$$

Next, in view of assumption $(L 0)_{\mathcal{A}_{h}} / 2^{0}$ for a given element $v \in \Lambda_{\mathcal{X}(e, \Omega)}$, there exists a sequence $\left\{\mathcal{R}_{e_{h_{n_{k}}}} v\right\}_{k \in N} \subset \mathcal{K}_{h_{n_{k}}}\left(e_{h_{n_{k}}}, \Omega\right)$ such that $\mathcal{R}_{e_{n_{n_{k}}}} v \rightarrow v$ strongly in $V_{h_{n_{k}}}(\Omega)$. Then after passing to the limit in (2.1) with $v_{h_{n}}=\mathcal{R}_{e_{h_{n_{k}}}}$ v as $k \rightarrow \infty$ we obtain, due to $(2.9),(2.10)$ and assumption $(H 1)_{B_{h}} / 6^{0}$,

$$
\left\langle\varepsilon \mathcal{A}(e) u_{\varepsilon}, v-v_{\varepsilon}\right\rangle_{V(\Omega)}+\left\langle\mathcal{B}(e) u_{\varepsilon}, v-u_{\varepsilon}\right\rangle_{W(\Omega)} \geq\left\langle f+B e, v-u_{\varepsilon}\right\rangle_{W(\Omega)}
$$

for any $v \in \Lambda_{\mathcal{K}(e, \Omega)}$.

On the other hand, by the density of $\Lambda_{\mathcal{K}(e, \Omega)},(2.11)$ holds for any $v \in \mathcal{K}(e, \Omega)$. This means, as $v \in \mathcal{K}(e, \Omega)$ is chosen arbitrarily, we get $u_{\varepsilon} \equiv u_{\varepsilon}(e)$ for any fixed $\varepsilon$, with $\frac{1}{2} \alpha_{B} \geq \varepsilon>h_{n}$. This proves the lemma 
In order to study the relation of optimal pairs to problems $\left(\mathcal{P}_{e h}\right)$ and $\left(\mathcal{P}_{e}\right)$, we need the additional assumptions

$(\mathbf{H 2})_{h}$

$$
\left\{\begin{aligned}
1^{0} \quad \text { The family }\left\{U_{a d}^{h}(\Omega)\right\}_{h \in(0,1)} \text { is compact in the following sense: } & \\
& \text { for any sequence }\left\{e_{h_{n}}\right\}_{n \in N} \subset U_{a d}^{h_{n}}(\Omega) \text { with } h_{n} \in(0,1) \text { and } \\
& h_{n} \rightarrow 0_{+} \text {there is a subsequence } h_{n_{k}} \rightarrow 0_{+} \text {and } e \in U_{a d}(\Omega) \text { such } \\
& \text { that } e_{h_{n_{k}}} \rightarrow e \text { strongly in } U^{h_{n_{k}}}(\Omega) . \\
2^{0} \quad \text { For any } e \in U_{a d}(\Omega) \text { and any sequence }\left\{h_{n}\right\}_{n \in N} \subset R, h_{n} \rightarrow 0_{+} & \\
& \text {there exists }\left\{e_{h_{n}}\right\}_{n \in N}, e_{h_{n}} \in U_{a d}^{h_{n}}(\Omega), \text { such that } e_{h_{n}} \rightarrow e \\
& \text { strongly in } U^{h_{n}}(\Omega) \\
3^{0} \quad & h_{n} \rightarrow 0_{+}, e_{h_{n}} \in U_{a d}^{h_{n}}(\Omega), e_{h_{n}} \rightarrow e \text { strongly in } U^{h_{n_{k}}}(\Omega), \\
& v_{h_{n}} \in V_{h_{n}}(\Omega), v_{h_{n}} \rightarrow v \text { strongly in } V_{h_{n}}(\Omega) \\
& \Rightarrow \mathcal{L}_{h_{n}}\left(e_{h_{n}}, v_{h_{n}}\right) \rightarrow \mathcal{L}(e, v) .
\end{aligned}\right.
$$

Theorem 4. Let assumptions $\left((H 1)_{\mathcal{A}_{h}},(H 1)_{B_{h}}\right)$ and $\left((L 0)_{\mathcal{A}_{h}},(H 2)_{B_{h}}\right)$ be satisfied. Further, let $\left[e_{\varepsilon h_{n}}^{*}, u_{\varepsilon h_{n}}\left(e_{\varepsilon h_{n}}^{*}\right)\right]$ be an optimal pair of problem $\left(\mathcal{P}_{\varepsilon h_{n}}\right)$ with $e_{\varepsilon h_{n}}^{*} \in U_{a d}^{h_{n}}(\Omega)$, $h_{n} \in(0,1)$ and $h_{n} \rightarrow 0_{+}, \frac{1}{2} \widehat{\alpha}_{B} \geq \varepsilon>h_{n}$. Then there exists a sequence $h_{n_{k}} \rightarrow 0_{+}$and a pair of subsequences

$$
\left[\left\{e_{e h_{n_{k}}^{*}}\right\}_{k \in N},\left\{u_{\varepsilon h_{n_{k}}}\left(e_{\varepsilon h_{n_{k}}^{*}}^{*}\right)\right\}_{k \in N}\right] \quad \text { of }\left[\left\{e_{\varepsilon h_{n}}^{*}\right\}_{n \in N},\left\{u_{\varepsilon h_{n}}\left(e_{e h_{n}}^{*}\right)\right\}_{n \in N}\right]
$$

and a pair of elements

$$
\left[e_{\varepsilon}^{*}, u_{\varepsilon}\left(e_{\varepsilon}^{*}\right)\right] \in U_{a d}(\Omega) \times \mathcal{K}\left(e_{\varepsilon}^{*}, \Omega\right)
$$

of problem $\left(\mathcal{P}_{\varepsilon}\right)$ such that

$$
\left[e_{\varepsilon h_{n_{k}}^{*}}^{*}, u_{\varepsilon h_{n_{k}}}\left(e_{\varepsilon h_{n_{k}}^{*}}\right)\right]_{k \in \mathbb{N}} \rightarrow\left[e_{\varepsilon}^{*}, u_{\varepsilon}\left(e_{\varepsilon}^{*}\right)\right] \text { in }\left[U^{h_{n_{k}}}(\Omega) \times V_{h_{n_{k}}}(\Omega)\right]
$$

as $h_{n_{k}} \rightarrow 0_{+}$, for a fixed positive number $\frac{1}{2} \widehat{\alpha}_{B} \geq \varepsilon>h_{n}$.

Proof. Assumption $\left((H 2)_{h}, 1^{0}\right)$ yields the existence of a sequence $\left\{e_{\varepsilon h_{n_{k}}}\right\}_{k \subset N} \subset$ $\left\{e_{c h_{n}}^{*}\right\}_{n \in N}$ and $e_{e}^{*} \in U_{a d}(\Omega)$ such that $e_{c h_{n_{k}}} \rightarrow e_{\varepsilon}^{*}$ strongly in $U(\Omega)$. By virtue of Lemma 2 we get $u_{\varepsilon h_{n_{k}}}\left(e_{\varepsilon h_{n_{k}}^{*}}\right) \rightarrow u_{\varepsilon}\left(e_{\varepsilon}^{*}\right)$ strongly in $V_{h_{n_{k}}}(\Omega)$. Then, due to assumption $(L 0)_{\mathcal{A}_{h}} / 2^{0}$ we have $u_{\varepsilon}\left(e_{\varepsilon}^{*}\right) \in \mathcal{K}\left(e_{\varepsilon}^{*}, \Omega\right)$. The definition of problem $\left(\mathcal{P}_{e h}\right)$ yields

$$
\mathcal{L}_{h_{n_{k}}}\left(e_{e h_{n_{k}}^{*}}^{*}, u_{\varepsilon h_{n_{k}}}\left(e_{e h_{n_{k}}^{*}}^{*}\right)\right) \leq \mathcal{L}_{h_{n_{k}}}\left(e_{e h_{n_{k}}}, u_{\varepsilon h_{n_{k}}}\left(e_{\varepsilon h_{n_{k}}}\right)\right)
$$

for arbitrary $e_{\varepsilon h_{n_{k}}} \in U_{a d}^{h_{n_{k}}}(\Omega)$. Let $\hat{e}_{\varepsilon} \in U_{a d}(\Omega)$ be given. One can find sequences $h_{n_{k}} \rightarrow 0_{+}$and $\left\{\hat{e}_{\varepsilon h_{n_{k}}}\right\}_{k \in N} \subset U_{a d}^{h_{n_{k}}}(\Omega)$ such that $\hat{e}_{e h_{n_{k}}} \rightarrow \hat{e}_{e}$ strongly in $U^{h_{n_{k}}}(\Omega)$ due to assumption $(H 2)_{h} / 2^{0}$. We have again $u_{c h_{n_{k}}}\left(\hat{e}_{e h_{n_{k}}}\right) \rightarrow u_{e}\left(\hat{e}_{\varepsilon}\right)$ strongly in $V_{h_{n_{k}}}(\Omega)$, and using (2.13) and assumption $(H 2)_{h} / 3^{0}$ we get $\mathcal{L}_{h_{n_{k}}}\left(e_{e}^{*}, u_{e}\left(e_{e}^{*}\right)\right) \leq \mathcal{L}_{h_{n_{k}}}\left(\widehat{e}_{\varepsilon}, u_{\varepsilon}\left(\widehat{e}_{\varepsilon}\right)\right)$ for any $\widehat{e}_{\varepsilon} \in U_{a d}(\Omega)$ and $\frac{1}{2} \hat{\alpha}_{B} \geq \varepsilon>0$ and the proof is finished

Problem $\left(\mathcal{P}_{0}\right)$ can be treated quite analogously and an appropriate variant of Lemma 2 and Theorem 4 for this case $(\varepsilon=0)$ is the following. 
Theorem 5. Let assumptions $(H 1)_{B_{h}}$ and $\left((H 2)_{h}, \varepsilon=0\right)$ be satisfied. Further, let $\left[e_{0 h_{n}}^{*}, u_{0 h_{n}}\left(e_{0 h_{n}}^{*}\right)\right]_{n \in \mathbf{N}}$ be an optimal pair of problem $\left(\mathcal{P}_{0 h_{n}}\right), e_{0 h_{n_{k}}}^{*} \in U_{a d}^{h_{n_{k}}}(\Omega), h_{n} \in$ $(0,1) \quad(n \in \mathbb{N})$ and $h_{n} \rightarrow 0_{+}$. Then there exists a pair of subsequences

$$
\left[\left\{e_{0 h_{n_{k}}}^{*}\right\}_{k \in N},\left\{u_{0 h_{n_{k}}}\left(e_{0 h_{n_{k}}^{*}}^{*}\right)\right\}_{k \in N}\right] \text { of }\left[\left\{e_{0 h_{n}}^{*}\right\}_{n \in N},\left\{u_{0 h_{n}}\left(e_{0 h_{n}}^{*}\right)\right\}_{n \in N}\right]
$$

and a pair of a elements

$$
\left[e_{0}^{*}, u\left(e_{0}^{*}\right)\right] \in U_{a d}(\Omega) \times \mathcal{O}\left(e_{0}^{*}, \Omega\right)
$$

such that

$$
\left[e_{0 h_{n_{k}}}^{*}, u_{0 h_{n_{k}}}\left(e_{0 h_{n_{k}}^{*}}\right)\right]_{k \in N} \rightarrow\left[e_{0}^{*}, u_{0}\left(e_{0}^{*}\right)\right] \text { in } U^{h_{n_{k}}}(\Omega) \times W_{h_{n_{k}}}(\Omega)
$$

as $h_{n_{k}} \rightarrow 0_{+}$.

We have shown that the sequence of an optimal pairs of approximate singular perturbations problems $\left(\mathcal{P}_{\varepsilon h}\right)$ converges to the solution of problem $\left(\mathcal{P}_{\varepsilon}\right)$, as $h_{n} \rightarrow 0_{+}$for a fixed positive number $\varepsilon>h$ (by virtue of (2.7)). On the other hand, the sequence of optimal pairs of approximate limit problems $\left(\mathcal{P}_{0 h}\right)$ converges to the solution of problem $\left(\mathcal{P}_{0}\right)$ as $h_{n} \rightarrow 0_{+}$.

However, the above results do not indicate that the sequence $\left\{\left[e_{\varepsilon h_{n}}^{*}, u_{c h_{n}}\left(e_{e h_{n}}^{*}\right)\right\}\right]_{n \in N}$ converges uniformly in $\varepsilon$ to the optimal pair $\left[e_{0}^{*}, u_{0}\left(e_{0}^{*}\right)\right]$ of problem $\left(\mathcal{P}_{0}\right)$ as $h_{n} \rightarrow 0_{+}$.

\section{Non-coercive limit problem}

We make the basic assumptions

$$
(\mathbf{H 2})_{\mathcal{A}} \begin{cases}1^{0} & \{\mathcal{K}(e, \Omega)\}_{e \in U_{\text {ad }}(\Omega)} \text { satisfies assumptions }(H 0) / 1^{0}, 2^{0} \\ 2^{0} & \{\mathcal{A}(e)\}_{e \in U_{a d}(\Omega) \subset \mathcal{E}_{V(\Omega)}\left(\alpha_{\mathcal{A}}, M_{\mathcal{A}}\right)} \\ 3^{0} & e_{n} \rightarrow e \text { strongly in } U(\Omega) \Rightarrow \mathcal{A}\left(e_{n}\right) \rightarrow \mathcal{A}(e) \text { in } L\left(V(\Omega), V^{*}(\Omega)\right)\end{cases}
$$

and

$(\mathbf{H 2})_{\mathcal{B}} \begin{cases}1^{0} & \{\mathcal{O}(e, \Omega)\}_{e \in U_{\mathrm{ad}}(\Omega)} \text { satisfies assumptions }(H 1) / 1^{0}, 2^{0} \\ 2^{0} & \{\mathcal{B}(e)\}_{e \in U_{\mathrm{ad}}(\Omega) \subset \mathcal{E}_{W(\Omega)}\left(0, M_{\mathcal{B}}\right)} \\ 3^{0} & e_{n} \rightarrow e \text { strongly in } U(\Omega) \Rightarrow \mathcal{B}\left(e_{n}\right) \rightarrow \mathcal{B}(e) \text { in } L\left(W(\Omega), W^{*}(\Omega)\right)\end{cases}$

We set

$$
\mathcal{W}(\Omega)=\left\{v \in W(\Omega):\langle\mathcal{B}(e) v, v\rangle_{W(\Omega)}=0 \text { for all } e \in U_{a d}(\Omega)\right\}
$$

By virtue of assumption $(H 2)_{B} / 2^{0}$, it is easy to see that $W(\Omega)$ is a closed subspace of $W(\Omega)$. We denote by $W(\Omega) / \mathcal{W}(\Omega)$ the factor (or quotient) space of classes

$$
\hat{v}=\{v+p: v \in W(\Omega) \text { and } p \in \mathcal{W}(\Omega)\}
$$


endowed with the norm

$$
\|\hat{v}\|_{W(\Omega) / W(\Omega)}=\inf _{p \in \mathcal{W}(\Omega)}\|v+p\|_{W(\Omega)}
$$

Let $W(\Omega)=\mathcal{N}(\Omega) \oplus \mathcal{W}(\Omega)$ be the orthogonal decomposition of $W(\Omega)$ by means of the scalar product $(\cdot, \cdot)_{W(\Omega)}$. Clearly, for $W(\Omega) / \mathcal{W}(\Omega)$ (being the space of the equivalence classes obtained from $W(\Omega)$ by indentifying all the elements of $\mathcal{W}(\Omega)$ )

$$
\|\hat{v}\|_{W(\Omega) / W(\Omega)}=\inf _{v \in \hat{v}}\|v\|_{W(\Omega)}^{2}=\inf _{\substack{v=p+q \in \dot{j} \\ p \in \mathcal{N}(\Omega), q \in W(\Omega)}}\left(\|q\|_{W(\Omega)}^{2}+\|p\|_{W(\Omega)}^{2}\right)=\|p\|_{W(\Omega)}^{2}
$$

holds and thus $W(\Omega) / \mathcal{W}(\Omega)$ is a Hilbert space. We define a bilinear form on $W(\Omega) / \mathcal{W}(\Omega)$ by means of the relation

$$
\langle\widehat{\mathcal{B}}(e), \hat{v}, \hat{z}\rangle_{W(\Omega) / W(\Omega)}=\langle\mathcal{B}(e) v, z\rangle_{W(\Omega)} \quad\left(v \in \hat{v}, z \in \hat{z}, e \in U_{a d}(\Omega)\right) .
$$

Moreover, we suppose that there is $\alpha_{\mathcal{B}}>0$ such that

$(\mathbf{H 2})_{\Pi}\langle\mathcal{B}(e) v, z\rangle_{W(\Omega)}+\left\|\Pi_{W} v\right\|_{W(\Omega)}^{2} \geq \alpha_{B}\|v\|_{W(\Omega)}^{2}$

for any $v \in W(\Omega)$ and $e \in U_{a d}(\Omega)$, where $\Pi_{W}$ is the projection of $W(\Omega)$ onto $\mathcal{W}(\Omega)$. Simultancously, the symmetry and bilinearity of $\langle\mathcal{B}(e) \cdot, \cdot\rangle_{W(\Omega)}$ yield

$$
\begin{aligned}
\langle\mathcal{B}(e) v, z\rangle_{W(\Omega)} & =\left\langle\mathcal{B}(e)\left(\Pi_{\mathcal{N}} v+\Pi_{\mathcal{W} v}\right), \Pi_{\mathcal{N}} z+\Pi_{W} z\right\rangle_{W(\Omega)} \\
& =\left\langle\mathcal{B}(e) \Pi_{\mathcal{N}} v, \Pi_{\mathcal{N}} z\right\rangle_{W(\Omega)}
\end{aligned}
$$

since the remaining terms are zero. This follows from (3.1) and from the Schwarz inequality

$$
\left|\langle\mathcal{B}(e) v, z\rangle_{W(\Omega)}\right| \leq\left(\langle\mathcal{B}(e) v, v\rangle_{W(\Omega)}\right)^{\frac{1}{2}}\left(\langle\mathcal{B}(e) z, z\rangle_{W(\Omega)}\right)^{\frac{1}{2}} .
$$

Furthermore, assumption $(H 2)_{\Pi}$ yields in virtue of (3.3) - (3.5)

$$
\langle\widehat{\mathcal{B}}(e), \widehat{v}, \widehat{z}\rangle_{W(\Omega) / W(\Omega)}=\left\langle\mathcal{B}(e) \Pi_{\mathcal{N}} v, \Pi_{\mathcal{N} v}\right\rangle_{W(\Omega)} \geq \alpha_{\mathcal{B}}\|\widehat{v}\|_{W(\Omega)}^{2}
$$

and therefore the bilinear form $\langle\widehat{\mathcal{B}}(e), \cdot\rangle_{W(\Omega) / W(\Omega)}$ is cocrcive on $W(\Omega) / \mathcal{W}(\Omega)$.

Now, we set

$$
\mathcal{M}(e, \Omega):=\operatorname{cl}\{\hat{v} \in W(\Omega) / \mathcal{W}(\Omega): \text { there exists } v \in \hat{v}, v \in \mathcal{O}(e, \Omega)\}
$$

for all $e \in U_{a d}(\Omega)$. We proceed now to set the following assumption:

$\left(\mathbf{H}_{2}\right)_{\mathcal{M}}\left\{\begin{array}{ll}1^{0} & e_{n} \rightarrow e \text { strongly in } U(\Omega) \Rightarrow \operatorname{Lim}_{n \rightarrow \infty} \mathcal{M}\left(e_{n}, \Omega\right)=\mathcal{M}(e, \Omega) . \\ & \left\{e_{n}\right\}_{n \in \mathbb{N}} \in U_{\text {ad }}(\Omega) \\ 2^{0} & e_{n} \rightarrow e \in U_{a d}(\Omega) \text { strongly in } U(\Omega) \\ & \hat{v}, \hat{v}_{n} \in W(\Omega) / W(\Omega)(n \in \mathbb{N}) \\ & \hat{v}_{n} \rightarrow \hat{v} \text { weakly in } W(\Omega) / \mathcal{W}(\Omega)\end{array}\right\} \Rightarrow \mathcal{L}(e, \hat{v}) \leq \liminf _{n \rightarrow \infty} \mathcal{L}\left(e_{n}, \hat{v}_{n}\right)$. 
Moreover, we introduce the annihilator $\mathcal{W}^{\perp}(\Omega)$ of $\mathcal{W}(\Omega)$ as

$$
\mathcal{W}^{\perp}(\Omega):=\left\{g \in W^{*}(\Omega):\langle g, v\rangle_{W(\Omega)}=0 \text { for all } v \in \mathcal{W}(\Omega)\right\}
$$

Thus $\mathcal{W}^{\perp}(\Omega) \subset W^{*}(\Omega)$ is the set of all continuous linear functionals on $W(\Omega)$ that vanish identically on $\mathcal{W}(\Omega)$. The elements of $\mathcal{W}^{\perp}(\Omega)$ generate in an obvious way continuous linear functionals on $W(\Omega) / W(\Omega)$.

In the following, we suppose that the function $\mathcal{L}: U_{a d}(\Omega) \times W(\Omega) / \mathcal{W}(\Omega) \rightarrow \mathbb{R}^{+}$ with $\mathcal{L}(e, \hat{v})=\mathcal{L}\left(e, \Pi_{\mathcal{N}} v\right)$ fulfils assumption $(H 2)_{\mathcal{M}} / 2^{0}$.

Perturbated state operator and perturbated cost function. For every $\varepsilon>0$ and for every $e \in U_{a d}(\Omega)$, there exists a unique $u_{\varepsilon}(e) \in \mathcal{K}(e, \Omega)$ such that

$$
\left\langle\varepsilon \mathcal{A}(e) u_{\varepsilon}(e)+\mathcal{B}(e) u_{\varepsilon}(e), v-u_{\varepsilon}(e)\right\rangle_{V(\Omega)} \geq\left\langle f+B e, v-u_{\varepsilon}(c)\right\rangle_{W(\Omega)}
$$

for all $v \in \mathcal{X}(\Omega)$ (as we obtain estimate (1.4) from assumptions $(H 2)_{\mathcal{A}} / 2^{0}$ and $\left.(H 2)_{B} / 2^{0}\right)$. Here we assume $(f+\mathrm{Be}) \in W^{*}(\Omega)$ with $e \in U_{a d}(\Omega)$. Moreover, we assume that the cost function $\mathrm{J}_{e}(e)=\mathcal{L}\left(e, u_{\varepsilon}(e)\right)$ satisfies hypotheses (E0).

Now, we define the perturbed optimization problem $\left(\mathcal{P}_{e}\right)$. Find a control $e_{e}^{*} \in U_{a d}(\Omega)$ such that

$$
\mathcal{J}_{\varepsilon}\left(e_{\varepsilon}^{*}\right)=\inf \mathcal{J}_{\varepsilon}(e) \quad\left(e \in U_{\mathrm{ad}}(\Omega)\right) .
$$

Limit state operator and limit cost function. The limit optimization problem will have the form

$\left(\mathcal{P}_{0}\right)$. Find a couple $\left[e_{0}^{*}, \hat{u}_{0}\left(e_{0}^{*}\right)\right] \in U_{a d}(\Omega) \times \mathcal{M}(e, \Omega)$ such that

$$
\hat{\mathcal{J}}_{0}\left(e_{\varepsilon}^{*}\right) \leq \hat{\mathcal{J}}_{0}(e) \quad \forall e \in U_{a d}(\Omega)
$$

with

$$
\widehat{\mathcal{J}}(e)=\mathcal{L}\left(e, \widehat{u}_{0}(e)\right)=\mathcal{L}\left(e, u_{0 \mathcal{N}}(e)\right)
$$

where $\hat{u}_{0}(e) \in \mathcal{M}(e, \Omega)$ such that

$$
\left\langle\widehat{\mathcal{B}}(e) \hat{u}_{0}(e), \hat{v}-\hat{u}_{0}(e)\right\rangle_{W(\Omega) / \mathcal{W}(\Omega)} \geq\left\langle f+B(e), \hat{v}-\hat{u}_{0}(e)\right\rangle_{W(\Omega) / \mathcal{W}(\Omega)}
$$

for any $\hat{v} \in \mathcal{M}(e, \Omega)$ and $u_{0}(e)=u_{0 \mathcal{N}}(e)+u_{0} w(e)$ with $u_{0 \mathcal{N}}(e) \in \mathcal{N}(\Omega)$ and $u_{0} w(e) \epsilon$ $\mathcal{W}(\Omega)$. Here we suppose that

$$
(f+B e) \in \mathcal{W}^{\perp}(\Omega) \quad \forall e \in U_{a d}(\Omega)
$$

and for the sake of simplicity we write $\langle f+B e, \hat{v}\rangle_{W(\Omega) / W(\Omega)}$ for any $\hat{v} \in W(\Omega) / W(\Omega)$ and $e \in U_{a d}(\Omega)$.

The following two theorems are valid. 
Theorem 6. Let assumptions $(H 2)_{\mathcal{A}}$ and $(H 2)_{B}$ be satisfied. Then there exists at least one solution to problem $\left(\mathcal{P}_{e}\right)$.

Proof. The proof is analogous to that of Theorem 1 and hence it is omitted

Theorem 7. Let assumptions $(H 2)_{\mathcal{A}},(H 2)_{B},(H 2)_{\Pi},(H 2)_{\mathcal{M}}$ and $(3.11)$ be satisfied. Then there exists at least one solution to problem $\left(\mathcal{P}_{0}\right)$..

Proof. If we rewrite all the situation to the factor-space terms, then we can see that the proof is again analogous to that of Theorem 1 and hence it is omitted

Therefore, by virtue of Theorems 6 and $7, u_{\varepsilon_{m}}(e)$ and $\hat{u}_{0}(e)$ are well determined elements of $V(\Omega)$ and $W(\Omega) / \mathcal{W}(\Omega)$, respectively. The relation between the solutions to problems $\left(\mathcal{P}_{0}\right)_{*}$ and $\left(\mathcal{P}_{e_{m}}\right)$, as $\varepsilon_{m} \rightarrow 0$ then follows from the following theorem.

Theorem 8. Let assumptions $(E 0),(H 2)_{\mathcal{A}},(H 2)_{B},(H 2)_{\Pi},(H 2)_{\mathcal{M}}$ and (3.11) be satisfied. Let $e_{e_{n}}^{*}$ be the solution of problem $\left(\mathcal{P}_{\varepsilon_{n}}\right)$, and $\varepsilon_{n} \rightarrow 0$. Then there exists $a$ subsequence $\left\{e_{n_{k}}^{*}\right\}_{k \in N}$ of $\left\{e_{\varepsilon_{n}}^{*}\right\}_{n \in N}$ and a solution $e_{0}^{*}$ of problem $\left(\mathcal{P}_{0}\right)$. such that

$$
\left.\begin{array}{r}
\varepsilon_{n_{k}} \rightarrow 0, e_{\varepsilon_{n_{k}}^{*}}^{*} \rightarrow e_{0}^{*} \text { strongly in } U(\Omega) \\
\hat{u}_{\varepsilon_{n_{k}}}\left(e_{\varepsilon_{n_{k}}^{*}}^{*}\right) \rightarrow \hat{u}_{0}\left(e_{0}^{*}\right) \text { weakly in } W(\Omega) / \mathcal{W}(\Omega) \\
\varepsilon_{n_{k}}\left\langle\mathcal{A}\left(e_{\varepsilon_{n_{k}}^{*}}^{*}\right) u_{\varepsilon_{n_{k}}}\left(e_{e_{n_{k}}^{*}}^{*}\right), u_{\varepsilon_{n_{k}}}\left(e_{\varepsilon_{n_{k}}^{*}}^{*}\right)\right\rangle_{V(\Omega)} \leq C \\
\hat{\mathcal{J}}_{e_{n_{k}}}\left(e_{\varepsilon_{n_{k}}^{*}}^{*}\right)=\inf \hat{\mathcal{J}}_{\varepsilon_{n_{k}}}(e) \Rightarrow \widehat{\mathcal{J}}_{0}\left(e_{0}^{*}\right)=\inf \widehat{\mathcal{J}}_{0}(e), e \in U_{a d}(\Omega) .
\end{array}\right\}
$$

Proof. The proof is analogous to that of Theorem 3. Analogously to estimates before (1.27) we have

$$
\varepsilon_{n} \alpha_{\mathcal{A}}\left\|u_{\varepsilon_{n}}\left(e_{\varepsilon_{n}}^{*}\right)\right\|_{V(\Omega)}^{2}+\alpha_{B}\left\|\Pi_{\mathcal{N}} u_{e_{n}}\left(e_{e_{n}}^{*}\right)\right\|_{W(\Omega)}^{2} \leq M_{\mathcal{N}}\left\|\Pi_{\mathcal{N} u_{e_{n}}}\left(e_{e_{n}}^{*}\right)\right\|_{W(\Omega)}+M
$$

and from (3.3)

$$
\varepsilon \alpha_{\mathcal{A}}\left\|u_{e_{n}}\left(e_{e_{n}}^{*}\right)\right\|_{V(\Omega)}^{2} \leq M_{\mathcal{A}} \quad \text { and } \quad\left\|u_{e_{n}}\left(e_{e_{n}}^{*}\right)\right\|_{W(\Omega) / W(\Omega)} \leq M_{W}
$$

Therefore, we can extract sequences $\left\{\varepsilon_{n_{k}}\right\}_{k \in N}$ and $\left\{\hat{u}_{\varepsilon_{n_{k}}}\left(e_{\varepsilon_{n_{k}}^{*}}\right)\right\}_{k \in N}$ such that

$$
\varepsilon_{n_{k}} \rightarrow 0 \quad \text { and } \quad \hat{u}_{e_{n_{k}}}\left(e_{\varepsilon_{n_{k}}^{*}}\right) \rightarrow \hat{u}_{0} \text { weakly in } W(\Omega) / \mathcal{W}(\Omega) \text {. }
$$

By the compactness of $U_{a d}(\Omega)$ one can suppose that $e_{e_{n_{k}}}^{*} \rightarrow e_{0}, e_{0} \in U_{a d}(\Omega)$. Moreover, due to assumption $(H 2) / 1^{0}$, for any element $v \in \mathcal{O}\left(e_{0}, \Omega\right)$ there exists a sequence $\left\{v_{k}\right\}_{k \in N} \subset \mathcal{K}\left(e_{e_{n_{k}}^{*}}, \Omega\right)$ such that

$$
v_{k} \rightarrow v \quad \text { strongly in } W(\Omega)
$$

Thus, by virtue of (3.15) and assumption $(H 2) / 2^{\circ}$, we obtain

$$
\begin{aligned}
\mid \varepsilon_{n_{k}} & \left\langle\mathcal{A}\left(e_{e_{n_{k}}^{*}}^{*}\right) u_{\varepsilon_{n_{k}}}\left(e_{e_{n_{k}}^{*}}^{*}\right), v_{k}\right\rangle_{V(\Omega)} \mid \\
& \leq \varepsilon_{n_{k}}\left(\left\langle\mathcal{A}\left(e_{e_{n_{k}}^{*}}^{*}\right) u_{e_{n_{k}}}\left(e_{e_{n_{k}}^{*}}^{*}\right), u_{\varepsilon_{n_{k}}}\left(e_{e_{n_{k}}^{*}}^{*}\right)\right\rangle_{V(\Omega)}\right)^{\frac{1}{2}}\left(\left\langle\mathcal{A}\left(e_{e_{n_{k}}^{*}}^{*}\right) v_{k}, v_{k}\right\rangle_{V(\Omega)}\right)^{\frac{1}{2}} \\
& \rightarrow 0
\end{aligned}
$$


and

$$
\begin{aligned}
&\left\langle\mathcal{B}\left(e_{\varepsilon_{n_{k}}}^{*}\right) u_{\varepsilon_{n_{k}}}\left(e_{\varepsilon_{n_{k}}}^{*}\right), v_{k}\right\rangle_{W(\Omega)}=\left\langle\hat{\mathcal{B}}\left(e_{\varepsilon_{n_{k}}^{*}}^{*}\right) \hat{u}_{\varepsilon_{n_{k}}}\left(e_{\varepsilon_{n_{k}}}^{*}\right), \hat{v}_{k}\right\rangle_{W(\Omega) / W(\Omega)} \\
& \rightarrow\left\langle\hat{\mathcal{B}}\left(e_{0}\right) \hat{u}_{*}, \hat{v}\right\rangle_{W(\Omega) / W(\Omega)} \\
&\left\langle f+B e_{\varepsilon_{n_{k}}}^{*}, \hat{v}_{k}\right\rangle_{W(\Omega) / W(\Omega)} \rightarrow\left\langle f+B e_{0}, \hat{v}\right\rangle_{W(\Omega) / W(\Omega)}
\end{aligned}
$$

On the other hand, by comparison with (1.31) we can write

$$
\liminf _{k \rightarrow \infty}\left\langle\hat{\mathcal{B}}\left(e_{e_{n_{k}}^{*}}^{*}\right) \hat{u}_{\varepsilon_{n_{k}}}\left(e_{\varepsilon_{n_{k}}^{*}}^{*}\right), \hat{u}_{\varepsilon_{n_{k}}}\left(e_{\varepsilon_{n_{k}}^{*}}^{*}\right)\right\rangle_{W(\Omega) / W(\Omega)} \geq\left\langle\hat{\mathcal{B}}\left(e_{0}\right) \hat{u}_{0}, \hat{u}_{0}\right\rangle_{W(\Omega) / W(\Omega)} .
$$

Next, use the inequality

$$
\begin{aligned}
\left\langle\varepsilon_{n_{k}}\right. & \left.\mathcal{A}\left(e_{\varepsilon_{n_{k}}^{*}}^{*}\right) u_{\varepsilon_{n_{k}}}\left(e_{\varepsilon_{n_{k}}^{*}}^{*}\right), v_{k}-u_{\varepsilon_{n_{k}}}\left(e_{\varepsilon_{n_{k}}^{*}}^{*}\right)\right\rangle_{V(\Omega)} \\
& +\left\langle\hat{\mathcal{B}}\left(e_{\varepsilon_{n_{k}}^{*}}^{*}\right) \hat{u}_{\varepsilon_{n_{k}}}\left(e_{\varepsilon_{n_{k}}^{*}}^{*}\right), \hat{v}_{k}\right\rangle_{W(\Omega) / W(\Omega)} \\
& -\left\langle f+B e_{\varepsilon_{n_{k}}}^{*}, \hat{v}_{k}-\hat{u}_{\varepsilon_{n_{k}}}\left(e_{\varepsilon_{n_{k}}^{*}}^{*}\right)\right\rangle_{W(\Omega) / W(\Omega)} \\
\geq & \left\langle\hat{\mathcal{B}}\left(e_{\varepsilon_{n_{k}}^{*}}^{*}\right) \hat{u}_{\varepsilon_{n_{k}}}\left(e_{\varepsilon_{n_{k}}^{*}}^{*}\right), \hat{u}_{\varepsilon_{n_{k}}}\left(e_{\varepsilon_{n_{k}}^{*}}^{*}\right)\right\rangle_{W(\Omega) / W(\Omega)} .
\end{aligned}
$$

From it and due to (3.16) - (3.19) and the definition of $\mathcal{M}\left(e_{0}, \Omega\right)$ we obtain

$$
\left\langle\widehat{\mathcal{B}}\left(e_{0}\right) \hat{u}_{0}, \hat{v}-\hat{u}_{0}\right\rangle_{W(\Omega) / W(\Omega)} \geq\left\langle f+B e_{0}, \hat{v}-\hat{u}_{0}\right\rangle_{W(\Omega) / W(\Omega)}
$$

for any $\hat{v} \in \mathcal{M}\left(e_{0}, \Omega\right)$. This yields $\hat{u}_{0}=\hat{u}_{0}\left(e_{0}\right)$ and consequently, for $k \rightarrow \infty$,

$$
\left.\begin{array}{c}
e_{\varepsilon_{n_{k}}} \rightarrow e_{0} \text { strongly in } U(\Omega) \\
\hat{u}_{\varepsilon_{n_{k}}}\left(e_{\varepsilon_{n_{k}}^{*}}\right) \rightarrow \hat{u}_{0}\left(e_{0}\right) \text { weakly in } W(\Omega) / \mathcal{W}(\Omega)
\end{array}\right\}
$$

(since the variational inequality (3.10) has a unique solution for $e \in U_{a d}(\Omega)$ ). Moreover, due to the strong convergence $v_{n} \rightarrow v$, and regarding (3.4) - (3.5), (3.7) and (1.2) we deduce from (3.20) that

$$
\begin{aligned}
\limsup _{k \rightarrow \infty} & \left\langle\hat{\mathcal{B}}\left(e_{\varepsilon_{n_{k}}}^{*}\right) \hat{u}_{\varepsilon_{n_{k}}}\left(e_{\varepsilon_{n_{k}}}^{*}\right), \hat{u}_{\varepsilon_{n_{k}}}\left(e_{\varepsilon_{n_{k}}}^{*}\right)\right\rangle_{W(\Omega) / W(\Omega)} \\
\quad \leq & \limsup _{k \rightarrow \infty}\left[\left\langle\hat{\mathcal{B}}\left(e_{\varepsilon_{n_{k}}}^{*}\right) \hat{u}_{\varepsilon_{n_{k}}}\left(e_{\varepsilon_{n_{k}}}^{*}\right), \hat{v}_{k}\right\rangle_{W(\Omega) / W(\Omega)}\right. \\
& \left.-\left\langle f+B e_{e_{n_{k}}}^{*}, \hat{v}_{k}-\hat{u}_{\varepsilon_{n_{k}}}\left(e_{\varepsilon_{n_{k}}}^{*}\right)\right\rangle_{W(\Omega) / W(\Omega)}\right] \\
= & \left\langle\hat{\mathcal{B}}\left(e_{0}\right) \hat{u}_{0}\left(e_{0}\right), \hat{v}\right\rangle_{W(\Omega) / W(\Omega)}-\left\langle f+B e_{0}, \hat{v}-\hat{u}_{0}\left(e_{0}\right)\right\rangle_{W(\Omega) / W(\Omega)}
\end{aligned}
$$

The last equality is a consequence of (3.14) - (3.15) and (3.17) - (3.18), and all relations in (3.23) hold for all $\hat{v} \in \mathcal{M}\left(e_{0}, \Omega\right)$. Therefore, taking $\hat{v}=\hat{u}\left(e_{0}\right) \in \mathcal{M}\left(e_{0}, \Omega\right)$ in (3.23), we obtain

$$
\begin{gathered}
\limsup _{k \rightarrow \infty}\left\langle\hat{\mathcal{B}}\left(e_{\varepsilon_{n_{k}}^{*}}^{*}\right) \hat{u}_{\varepsilon_{n_{k}}}\left(e_{\varepsilon_{n_{k}}^{*}}^{*}\right), \hat{u}_{\varepsilon_{n_{k}}}\left(e_{\varepsilon_{n_{k}}^{*}}^{*}\right)\right\rangle_{W(\Omega) / W(\Omega)} \\
\leq\left\langle\hat{\mathcal{B}}\left(e_{0}\right) \hat{u}_{0}\left(e_{0}\right), \hat{u}_{0}\left(e_{0}\right)\right\rangle_{W(\Omega) / \mathcal{W}(\Omega)} .
\end{gathered}
$$


Thus, due to (3.19), (3.22) and (3.24),

$$
\begin{gathered}
\lim _{k \rightarrow \infty}\left\langle\widehat{\mathcal{B}}\left(e_{\varepsilon_{n_{k}}^{*}}^{*}\right) \hat{u}_{e_{n_{k}}}\left(e_{\varepsilon n_{k}}^{*}\right), \hat{u}_{e_{n_{k}}}\left(e_{e_{n_{k}}^{*}}^{*}\right)\right\rangle_{W(\Omega) / W(\Omega)} \\
=\left\langle\widehat{\mathcal{B}}\left(e_{0}\right) \hat{u}_{0}\left(e_{0}\right), \hat{u}_{0}\left(e_{0}\right)\right\rangle_{W(\Omega) / W(\Omega)}
\end{gathered}
$$

holds. On the other hand, we set

$$
\begin{aligned}
\mathcal{N}_{\varepsilon_{n}}(e) \equiv & \left\langle\widehat{\mathcal{B}}(e)\left(\hat{u}_{e_{n}}(e)-\hat{u}_{0}(e)\right), \hat{u}_{\varepsilon_{n}}(e)-\hat{u}_{0}(e)\right\rangle_{W(\Omega) / W(\Omega)} \\
\leq & \left\langle\mathcal{B}(e) \hat{u}_{e_{n}}(e), \hat{u}_{e_{n}}(e)-\hat{u}_{0}(e)\right\rangle_{W(\Omega) / W(\Omega)} \\
& -\left\langle f+B(e), \hat{u}_{e_{n}}(e)-\hat{u}_{0}(e)\right\rangle_{W(\Omega) / W(\Omega)} .
\end{aligned}
$$

Then we obtain with the help of (3.20)

$$
\begin{aligned}
&\left\langle\widehat{\mathcal{B}}(e) \hat{u}_{e_{n_{k}}}(e), \hat{u}_{e_{n_{k}}}(e)\right\rangle_{W(\Omega) / W(\Omega)} \\
& \leq\left\langle\varepsilon_{n_{k}} \mathcal{A}(e) u_{e_{n_{k}}}(e), u_{e_{n_{k}}}(e)\right\rangle_{V(\Omega)}+\left\langle\widehat{\mathcal{B}}(e) \hat{u}_{\varepsilon_{n_{k}}}(e), \hat{u}_{\varepsilon_{n_{k}}}(e)\right\rangle_{W(\Omega) / W(\Omega)} \\
& \leq\left\langle f+B e, \hat{u}_{e_{n_{k}}}(e)-\hat{v}\right\rangle_{W(\Omega) / W(\Omega)} \\
&+\left\langle\varepsilon_{n_{k}} \mathcal{A}(e) u_{\varepsilon_{n_{k}}}(e), v\right\rangle_{V(\Omega)}+\left\langle\widehat{\mathcal{B}}(e) \hat{u}_{\varepsilon_{n_{k}}}(e), \hat{v}\right\rangle_{W(\Omega) / W(\Omega)}
\end{aligned}
$$

for $v$ fixed in $\mathcal{K}(e, \Omega)$. From it one has

$$
\begin{aligned}
\mathcal{N}_{e_{n_{k}}}(e) \leq\langle & \left.+B(e), \hat{u}_{0}(e)-\hat{v}\right\rangle_{W(\Omega) / W(\Omega)} \\
& +\left\langle\varepsilon_{n_{k}} \mathcal{A}(e) u_{e_{n_{k}}}(e), v\right\rangle_{V(\Omega)}+\left\langle\hat{\mathcal{B}}(e) \hat{u}_{e_{n_{k}}}(e), \hat{v}-\hat{u}_{0}(e)\right\rangle_{W(\Omega) / W(\Omega)}
\end{aligned}
$$

which give due to (1.29), (3.14), (3.17) and (3.22)

$$
\begin{aligned}
& \limsup _{k \rightarrow \infty} \mathcal{N}_{e_{n}}(e) \\
& \quad \leq\left\langle f+B(e), \hat{u}_{0}(e)-\hat{v}\right\rangle_{W(\Omega) / W(\Omega)}+\left\langle\widehat{\mathcal{B}}(e) \hat{u}_{0}(e), \hat{v}-\hat{u}_{0}(e)\right\rangle_{W(\Omega) / W(\Omega)}
\end{aligned}
$$

for any $\hat{v} \in \mathcal{M}\left(e_{0}, \Omega\right)$ and $e \in U_{a d}(\Omega)$. Note that (3.27) is also true for $\hat{v}=\hat{u}_{0}(e)$, hence $\lim \sup _{k \rightarrow \infty} \mathcal{N}_{e_{n}}(e) \leq 0$ and from this estimate it follows that

$$
\hat{u}_{e_{n_{k}}}(e) \rightarrow \hat{u}_{0}(e) \text { strongly in } W(\Omega) / \mathcal{W}(\Omega) \text { for all } e \in U_{a d}(\Omega)
$$

We may write $\widehat{\mathcal{J}}_{\varepsilon_{n_{k}}}\left(e_{\varepsilon_{n_{k}}}^{*}\right) \leq \widehat{\mathcal{J}}_{e_{n_{k}}}(e)$ for all $e \in U_{a d}(\Omega)$ (which derives from the definition of $e_{\varepsilon_{n}}^{*}$ ). This means that due to assumption $(E 0) / 1^{0}$

$$
\begin{aligned}
& \limsup \widehat{\mathcal{J}}_{\varepsilon_{n_{k}}}\left(e_{\varepsilon_{n_{k}}^{*}}^{*}\right) \leq \hat{\mathcal{J}}_{0}(e) \text { for all } e \in U_{a d}(\Omega) \\
& \quad \Rightarrow \limsup _{k \rightarrow \infty} \widehat{\mathcal{J}}_{\varepsilon_{n_{k}}}\left(e_{\varepsilon_{n_{k}}^{*}}^{*}\right) \leq \inf _{e \in U_{a d}(\Omega)} \widehat{\mathcal{J}}_{0}(e)=\widehat{\mathcal{J}}_{0}\left(e_{0}^{*}\right) .
\end{aligned}
$$

On the other hand, due to assumption $(H 2)_{\mathcal{M}} / 2^{0}$ and (3.22) we get

$$
\liminf _{k \rightarrow \infty} \hat{\mathcal{J}}_{e_{n_{k}}}\left(e_{e_{n_{k}}^{*}}\right) \geq \mathcal{L}\left(e_{0}, \hat{u}_{0}\left(e_{0}\right)\right)=\hat{\mathcal{J}}_{0}\left(e_{0}\right) .
$$

Finally, comparing (3.30) with (3.29) we obtain $\widehat{\mathcal{J}}_{0}\left(e_{0}\right) \leq \widehat{\mathcal{J}}_{0}\left(e_{0}^{*}\right)$. Hence we see that necessarily $e_{0}=e_{0}^{*}$ and from (3.29) and (3.30) we also get (3.12) 4 which ends the proof 


\section{Application. The membrane approximation to the plate with inner obstacle (a case with coercive limit problem)}

The plate model corresponds to a plate subjected to stretching forces in the $(x, y)$ plane. In many practical applications, plates are in a state of initial membrane stress. When subsequently subjected to transverse pressure loads, their structural behaviour and response can be entirely different from plates which are free from such intcrnal stresses.

Let us consider a homogeneous isotropic Kirchhoff plate with small rigidity and with inner obstacle. The equilibrium position of the plate constrained to lie above an obstacle (rigid frictionless surface located at a distance $\mathcal{S}=\mathcal{S}(x, y)$ under the middle plain of the plate). The plate has a constant thickness $2 \varepsilon H_{\text {plate }}$. We assume that the midplane of the plate occupies a given bounded, convex and simply connected domain $\Omega \subset \mathbb{R}^{2}$ with a piecewise smooth boundary. The material constant $E$ (the Young modulus of elasticity) and a variable distributed load $q(x, y)$ (externally applied pressure) and rigid frictionless obstacle $\mathcal{S}(x, y)$ may be viewed as a design variable. To simplify notation they are denoted as a design vector $\mathrm{e}=[E, q, S]^{T}$.

We will consider physical situation in which the transverse displacement of the thin homogeneous isotropic plate is constrained by presence of a inner stiff punch (rigid frictionless inner obstacle).

Let the plate be simply supported at the boundary $\partial \Omega$. Therefore we assume $V(\Omega):=H^{2}(\Omega) \cap H_{0}^{1}(\Omega)$ and $W(\Omega):=H_{0}^{1}(\Omega)$. Here the set of kinematically admissible virtual displacements is defined as

$$
\mathcal{K}_{e}(\mathcal{S}, \Omega)=\left\{v \in V(\Omega): v \geq \mathcal{S}(x, y)+\varepsilon H_{\text {plate }} \text { on } \Omega\right\} \quad\left(\mathcal{S} \in U_{a d}^{S_{d}}(\Omega)\right)
$$

Moreover, in the space $W(\Omega)$ we consider the convex and closed set

$$
\mathcal{O}_{\varepsilon}(\mathcal{S}, \Omega)=\left\{v \in W(\Omega): v \geq \mathcal{S}(x, y)+\varepsilon H_{\text {mem }} \text { a.e. in } \Omega\right\} \quad\left(\mathcal{S} \in U_{a d}^{\mathcal{S}_{d}}(\Omega)\right) .
$$

Consider for the plate the design space $U(\Omega)$ and the admissible design set $U_{a d}(\Omega)$ as

$$
U(\Omega) \equiv R \times C(\bar{\Omega}) \times H^{2}(\Omega) \quad \text { and } \quad U_{a d}(\Omega)=U_{a d}^{E}(\Omega) \times U_{a d}^{q}(\Omega) \times U_{a d}^{\mathcal{S}}(\Omega)
$$

with

$$
\begin{aligned}
& U_{a d}^{E}(\Omega)=\left\{E \in \mathbb{R}^{+}: c_{1} \leq E \leq c_{2 E}\right\} \\
& U_{a d}^{q}(\Omega)=\left\{q \in W_{\infty}^{1}(\Omega) \mid \begin{array}{l}
c_{1 q} \leq q \leq c_{2 q} \text { a.e. in } \Omega \\
\left|\frac{\partial q}{\partial x}\right| \leq c_{x},\left|\frac{\partial q}{\partial y}\right| \leq c_{y}, \int_{\Omega} q d \Omega=c_{3 q}
\end{array}\right\} \\
& U_{a d}^{\mathcal{S}}(\Omega)= \begin{cases}\left.\mathcal{S} \in H^{2+\eta}(\Omega) \mid \begin{array}{l}
-M_{\max } \leq \mathcal{S} \leq-M_{\min } \\
\|\mathcal{S}\|_{H^{2+\eta}(\Omega)} \leq c_{S} \text { on } \Omega, \varepsilon H_{\text {plate }}+\mathcal{S}(\partial \Omega)<0
\end{array}\right\}\end{cases}
\end{aligned}
$$

where $c_{1 E}, c_{2 E}, c_{1 q}, c_{2 q}, c_{3 q}$ and $c_{x}, c_{y}, \eta, M_{\min }, M_{\max }, c_{S}$ are given positive constants such that $U_{a d}(\Omega)$ is non-empty and $\left(\varepsilon H_{\text {plate }}+\left(-M_{\min }\right)\right)<0$ on $\Omega$. 
Let $F_{a}$ and $\left(x_{a}, y_{a}\right) \in \bar{\Omega} \quad(a=1,2, \ldots, M)$ be given constants and points, respectively, and let $q \in L_{1}(\Omega)$. Define the virtual work of external loads by the formula

$$
\langle\mathcal{J}(q), v\rangle_{W(\Omega)}=\sum_{a=1}^{M} F_{a} v\left(x_{a}, y_{a}\right)+\int_{\Omega} q v d \Omega \quad(v \in V(\Omega))
$$

It represents $\mathcal{J}(q) \in W^{*}(\Omega)$, because of the continuous embedding $V(\Omega) \subset C(\bar{\Omega})$. Let us consider the cost functional to the optimal control problem in the form

$$
\mathcal{L}:[e, v] \rightarrow \int_{\Omega}\left[v-z_{d}\right]^{2} d \Omega
$$

(The cost functional corresponds with adjusting the deflection to a prescribed function $\left.z_{d}\right)$. We define on the open set $\Omega$ the bilinear forms $a(E, \cdot, \cdot)$ and $b(E, \cdot, \cdot)$ by the relations

$$
\begin{aligned}
\langle\mathcal{A}(E) v, z\rangle_{V(\Omega)} \\
\quad: \equiv a(E, v, z) \\
\quad: \equiv \int_{\Omega}\left[\mathcal{N}_{x x}(v), \mathcal{N}_{y y}(v), \mathcal{N}_{x y}(v)\right]\left[Q_{\mathcal{A}}(E)\right]\left[\mathcal{N}_{x x}(z), \mathcal{N}_{y y}(z), \mathcal{N}_{x y}(z)\right]^{T} d \Omega
\end{aligned}
$$

for all $v, z \in V(\Omega), \mathcal{A}(E): V(\Omega) \rightarrow V^{*}(\Omega)$, and

$$
\begin{aligned}
\langle\mathcal{B}(E) v, z\rangle_{W(\Omega)} & : \equiv b(E, v, z) \\
& : \equiv \int_{\Omega}\left[Q_{\mathcal{B}}(E)\right]\left(\mathcal{N}_{x}(v) \mathcal{N}_{x}(z)+\mathcal{N}_{y}(v) \mathcal{N}_{y}(z)\right) d \Omega
\end{aligned}
$$

for $v, z \in W(\Omega), \mathcal{B}(E): W(\Omega) \rightarrow W^{*}(\Omega)$, where $Q_{\mathcal{B}}(E)>0$ is a constant depending on the elastic properties of the membrane $\left(Q_{B}(E)\right.$ is a scalar factor proportional to $\left.E\right)$ and

$$
\left\{\begin{aligned}
{\left[Q_{\mathcal{A}}(E)\right] } & =\frac{2 E H_{\text {plate }}^{3}}{3\left(1-\nu^{2}\right)}\left[\begin{array}{ccc}
1 & \nu & 0 \\
\nu & 1 & 0 \\
0 & 0 & \frac{1-\nu}{2}
\end{array}\right], \nu \text { - Poisson ratio, } \frac{1}{2}>\nu>0 \\
Q_{B}(E) & =\frac{E H_{\text {mem }}}{12\left(1-\nu^{2}\right)} \\
\mathcal{N}_{x x}(v) & =\frac{\partial^{2} v}{\partial x^{2}}, \mathcal{N}_{y y}(v)=\frac{\partial^{2} v}{\partial y^{2}}, \mathcal{N}_{x y}(v)=\frac{\partial^{2} v}{\partial x \partial y} \\
\mathcal{N}_{x}(v) & =\frac{\partial v}{\partial x}, \mathcal{N}_{y}(v)=\frac{\partial v}{\partial y}
\end{aligned}\right.
$$

This is the system of strain-displacement relations for the linear theory of plate (or membrane) such that the deformation operators belong to the spaces $L\left(V(\Omega), L_{2}(\Omega)\right.$ ) or $L\left(W(\Omega), L_{2}(\Omega)\right)$.

The subspace $\mathcal{R}(\Omega) \subset V(\Omega)$ is the set of rigid body motion of the plate

$$
\mathcal{R}(\Omega):=\left\{v \in V(\Omega):\langle\mathcal{A}(E) v, v\rangle_{V(\Omega)}=0\right\} .
$$


The properties of the matrix $\left[Q_{\mathcal{A}}(E)\right]$ imply,

$$
\langle\mathcal{A}(E) v, z\rangle_{V(\Omega)}=\langle\mathcal{A}(E) z, v\rangle_{V(\Omega)} \quad \forall v, z \in V(\Omega), E \in U_{a d}^{E}(\Omega)
$$

and the existence of a constant $c_{\mathcal{A}}$ such that

$$
\begin{aligned}
& \langle\mathcal{A}(E) v, v\rangle_{V(\Omega)} \\
& \quad \geq c_{\mathcal{A}}\left[\left\|\mathcal{N}_{x x}(v)\right\|_{L_{2}(\Omega)}^{2}+\left\|\mathcal{N}_{y y}(v)\right\|_{L_{2}(\Omega)}^{2}+\left\|\mathcal{N}_{x y}(v)\right\|_{L_{2}(\Omega)}^{2}+\left\|\mathcal{N}_{y x}(v)\right\|_{L_{2}(\Omega)}^{2}\right] .
\end{aligned}
$$

Let $P_{V}(\Omega)$ be the subspace of all possible (virtual) rigid body displacements of the middle plane, i.e.

$$
P_{V}(\Omega):=\left\{\begin{array}{l|l}
v \in V(\Omega) & \begin{array}{l}
\left\|\mathcal{N}_{x x}(v)\right\|_{L_{2}(\Omega)}^{2}+\left\|\mathcal{N}_{y y}(v)\right\|_{L_{2}(\Omega)}^{2}+ \\
\left\|\mathcal{N}_{x y}(v)\right\|_{L_{2}(\Omega)}^{2}+\left\|\mathcal{N}_{y x}(v)\right\|_{L_{2}(\Omega)}^{2}=0
\end{array}
\end{array}\right\}
$$

Lemma 3. Let $v \in H^{2}(\Omega)$ and

$$
\left\|\mathcal{N}_{x x}(v)\right\|_{L_{2}(\Omega)}^{2}+\left\|\mathcal{N}_{y y}(v)\right\|_{L_{2}(\Omega)}^{2}+\left\|\mathcal{N}_{x y}(v)\right\|_{L_{2}(\Omega)}^{2}+\left\|\mathcal{N}_{y x}(v)\right\|_{L_{2}(\Omega)}^{2}=0 .
$$

Then $P_{V}(\Omega)=\{0\}$, i.e. $P_{V}(\Omega)$ reduces to the zero element. which

Proof. The regularization of the displacement $v$ gives an element $v^{h} \in \mathcal{E}(\bar{\Omega})$ for

$$
\left.\begin{array}{l}
\mathcal{N}_{x x}\left(v^{h}\right)=\left[\mathcal{N}_{x x}(v)\right]^{h}=0 \\
\mathcal{N}_{y y}\left(v^{h}\right)=\left[\mathcal{N}_{y y}(v)\right]^{h}=0 \\
\mathcal{N}_{x y}\left(v^{h}\right)=\left[\mathcal{N}_{x y}(v)\right]^{h}=0
\end{array}\right\}
$$

holds for every domain $\widehat{\Omega}$ such that $\overline{\widehat{\Omega}} \subset \Omega$, provided that $h$ is sufficiently small $(h<$ $\operatorname{dis}(\overline{\widehat{\Omega}}, \partial \Omega))$. Then from condition (4.6) we conclude that $v^{h} \rightarrow v$ in $L_{2}(\Omega)$ as $h \rightarrow 0$ and the finite-dimensional subspaces are closed in $L_{2}(\Omega)$. We conclude that $v^{h}$ is a linear polynomial in every interior subdomain $\widehat{\Omega}, \overline{\hat{\Omega}} \subset \Omega$ and thus throughout in $\Omega$. The homogeneous Dirichlet boundary value condition $\partial \Omega_{u}$, however, yields $v=0$. (The plate is fixed at $\partial \Omega$ in such a manner that it cannot translate in the $z$-axis, and then it can only rotate.) On the other hand, the definition of $\mathcal{R}(\Omega)$, inequality (4.5) and Lemma 3 imply that $\mathcal{R}(\Omega)=\{0\}$. We have

$$
\left.\begin{array}{l}
\mathcal{A}(E) \in \mathcal{E}_{V(\Omega)}\left(\alpha_{\mathcal{A}}, M_{\mathcal{A}}\right) \\
\mathcal{B}(E) \in \mathcal{E}_{W(\Omega)}\left(\alpha_{\mathcal{B}}, M_{\boldsymbol{B}}\right)
\end{array}\right\} \quad\left(E \in U_{a d}^{E}(\Omega)\right)
$$

(The systems of operators $\mathcal{A}(E)$ and $\mathcal{B}(E)\left(E \in U_{a d}^{E}(\Omega)\right)$ satisfy assumptions $(H 0) / 3^{0}$ and $\left.(H 1) / 3^{0}\right)$. The estimates

$$
\left.\begin{array}{l}
\left|\left\langle\mathcal{A}\left(E_{n}\right) v-\mathcal{A}(E) v, z\right\rangle_{V(\Omega)}\right| \leq M_{\mathcal{A}}\left|E_{n}-E\right|\|v\|_{V(\Omega)}\|z\|_{V(\Omega)} \\
\left|\left\langle\mathcal{B}\left(E_{n}\right) v-\mathcal{B}(E) v, z\right\rangle_{W(\Omega)}\right| \leq M_{\mathcal{B}}\left|E_{n}-E\right|\|v\|_{W(\Omega)}\|z\|_{W(\Omega)}
\end{array}\right\}
$$

are easy to obtain and assumptions $(H 0) / 4^{0}$ and $(H 1) / 4^{0}$ follow. 
Lemma 4. For any $\mathcal{S} \in U_{a d}^{\mathcal{S}}(\Omega)$ the set $\mathcal{K}_{e}(S, \Omega)$ is a closed and convex subset of $V(\Omega)$ and

$$
\mathcal{S}_{n} \rightarrow \mathcal{S} \text { strongly } C(\bar{\Omega}) \text { for } \mathcal{S}, \mathcal{S}_{n} \in U_{a d}^{\mathcal{S}}(\Omega) \Rightarrow \mathcal{K}_{\varepsilon}(\mathcal{S}, \Omega)=\operatorname{Lim}_{n \rightarrow \infty} \mathcal{K}_{e}\left(\mathcal{S}_{n}, \Omega\right)
$$

Proof. The form of $\mathcal{K}_{e}(\mathcal{S}, \Omega)$ follows directly from its definition. If $v_{n} \in \mathcal{K}_{e}\left(\mathcal{S}_{n}, \Omega\right)$, $\mathcal{S}_{n} \rightarrow S$ in $C(\bar{\Omega})$ and $v_{n} \rightarrow v$ weakly in $H^{2}(\Omega)$, then $v_{n} \rightarrow v$ strongly in $C(\bar{\Omega})$ and the inequality for the limit remains valid.

For any $v \in \mathcal{K}_{e}(\mathcal{S}, \Omega)$ there exits a sequence $\left\{v_{n}\right\}_{n \in N}$ such that $v_{n} \in V(\Omega), v_{n} \in$ $\mathcal{K}_{e}\left(\mathcal{S}_{n}, \Omega\right)$ for $n$ sufficiently great, and $v_{n} \rightarrow v$ strongly in $V(\Omega)$, as $n \rightarrow \infty$. Indeed, let us define $\theta=v-\left(\mathcal{S}+\varepsilon H_{\text {plate }}\right)$ so that $\theta \in C(\bar{\Omega}), \theta \geq 0$ in $\bar{\Omega}$ and $\vartheta_{n}=\left(\mathcal{S}_{n}+\right.$ $\mathcal{S})-\theta=\mathcal{S}_{n}-v+\varepsilon H_{\text {plate }}, \widetilde{\mathcal{O}}_{n}=\left\{[x, y] \in \Omega: \vartheta_{n}(x, y) \geq \frac{\mathcal{Q}}{2}\right\}$, where the constant $\mathcal{Q}$ is $\left(\varepsilon H_{\text {plate }}+\left(-M_{\min }\right)\right)<0$, due to the definition of $U_{a d}^{S}(\Omega)$. There exists an open set $\tilde{\mathcal{O}} \subset \overline{\widetilde{\mathcal{O}}} \subset \Omega$ such that

$$
\tilde{\mathcal{O}}_{n} \subset \tilde{\mathcal{O}} \quad(n \in \mathbb{N})
$$

To see this, we realise that

$$
\vartheta_{n}=\varepsilon H_{\text {plate }}+S_{n} \leq \mathcal{Q}
$$

on the boundary $\partial \Omega$. The continuity of $\vartheta_{n}$ and the constraints $\left|\frac{\partial \mathcal{S}_{n}}{\partial x}\right| \leq c_{x}$ and $\left|\frac{\partial S_{n}}{\partial y}\right| \leq c_{y}$ imply that $\bigcup_{n=1}^{\infty} \widetilde{\mathcal{O}}_{n} \subset \Omega$ and (4.7) follows. Obviously, there exists a function $\xi \in$ $C^{\infty}(\bar{\Omega})$ such that $\xi(x, y)=1$ for any $[x, y] \in \overline{\mathcal{O}}$ and $\xi(x, y)=0$ for $[x, y] \in \partial \Omega$, $0 \leq \xi(x, y) \leq 1$ for $[x, y] \in \Omega$. Let us set $v_{n}=v+\left\|\mathcal{S}_{n}-\mathcal{S}\right\|_{L_{\infty}(\Omega)} \xi$. Then $v_{n} \in V(\Omega)$ and $\left\|v-v_{n}\right\|_{V(\Omega)}=\left\|S_{n}-S\right\|_{L_{\infty}(\Omega)}\|\xi\|_{H^{2}(\Omega)} \rightarrow 0$ as $n \rightarrow \infty$. We can show that there exists $n_{0}>0$ such that for $n>n_{0}$

$$
v_{n} \geq \varepsilon H_{\text {plate }}+\mathcal{S}_{n} \text { in } \bar{\Omega} \Rightarrow v_{n} \in \mathcal{K}_{e}(\mathcal{S}, \Omega) .
$$

Indeed, if $[x, y] \in \widetilde{\mathcal{O}}$, then one has

$$
v_{n}=v+\left\|\mathcal{S}_{n}-\mathcal{S}\right\|_{L_{\infty}(\Omega)} \geq v+\left(\mathcal{S}_{n}-\mathcal{S}\right) \geq \varepsilon H_{\text {plate }}+\mathcal{S}_{n}
$$

On the other hand, if $[x, y] \in \bar{\Omega} \backslash \widetilde{\mathcal{O}}$, then we can write

$$
v_{n} \geq \varepsilon H_{\text {plate }}+\mathcal{S}+\theta+\left|\mathcal{S}_{n}-\mathcal{S}\right| \xi .
$$

Since $[x, y] \notin \widetilde{\mathcal{O}},[x, y] \notin \widetilde{O}_{n}$ for any $n$ and $\vartheta_{n} \leq \frac{\text { const }}{2}$ so that

$$
\left(\mathcal{S}_{n}-\mathcal{S}\right)-\theta \leq \frac{\mathrm{const}}{2} \quad \text { and } \quad-\frac{\text { const }}{2} \xi+(1-\xi) \theta \leq \theta+\left|\mathcal{S}_{n}-\mathcal{S}\right| \xi
$$

Inserting (4.9) into (4.8) we obtain $v_{n} \geq \varepsilon H_{\text {plate }}+\mathcal{S}+\Im$, where $\Im=-\frac{\text { const }}{2} \xi+(1-$ $\xi) \theta$. The function $\Im$ is continuous and attains a positive minimum $M=\Im\left(\left[x_{*}, y_{*}\right]\right)=$ $\min _{\bar{\Omega} \backslash \tilde{\mathcal{O}}} \Im>0$ in the compact set $\bar{\Omega} \backslash \tilde{\mathcal{O}}$. Indeed, let $\xi\left(x_{*}, y_{*}\right)=0$. Then $\left[x_{*}, y_{*}\right] \in \partial \Omega$ and $\Im\left(x_{*}, y_{*}\right)=\theta\left(x_{*}, y_{*}\right)=-\left(\varepsilon H_{\text {plate }}+\mathcal{S}\left(x_{*}, y_{*}\right)\right) \geq-$ const $>0$. If $\xi\left(x_{*}, y_{*}\right)>0$, then one has $\Im\left(x_{*}, y_{*}\right) \geq-\frac{\text { const }}{2} \xi\left(x_{*}, y_{*}\right)>0$. There exists $n_{0}(M)$ such that, for $n>n_{0}(M)$, $\left\|\mathcal{S}_{n}-\mathcal{S}\right\|_{L_{\infty}{ }^{-}(\Omega)} \leq \cdot M$. This means that $\Im(x, y) \geq \Im\left(x_{*}, y_{*}\right) \geq .\left\|\mathcal{S}_{n}-\mathcal{S}\right\|_{L_{\infty}(\Omega)} \geq\left(\mathcal{S}_{n}-\mathcal{S}\right)$ so that $v_{n}(x, y) \geq \varepsilon H_{\text {plate }}+\mathcal{S}_{n}(x, y), n>n_{0}(M)$. Thus the proof of Lemma 4 is completed 
Lemma 5. For any $\mathcal{S} \in U_{\text {ad }}^{S}(\Omega)$ the set $\mathcal{O}(\mathcal{S}, \Omega)$ is a closed and convex subset of $W(\Omega)$ and

$$
\mathcal{S}_{n} \rightarrow \mathcal{S} \text { in } C(\bar{\Omega}) \text { for } \mathcal{S}, \mathcal{S}_{n} \in U_{a d}^{S}(\Omega) \Rightarrow \mathcal{O}_{\varepsilon}(\mathcal{S}, \Omega)=\operatorname{Lim}_{n \rightarrow \infty} \mathcal{O}_{\varepsilon}\left(\mathcal{S}_{n}, \Omega\right) \text {. }
$$

Proof. The closedness follows from the Lebesgue Theorem. The convexity is immediate. Let $\mathcal{S}_{n} \in U_{\text {ad }}^{\mathcal{S}}(\Omega)$ with $\mathcal{S}_{n} \rightarrow \mathcal{S}$ strongly in $C(\bar{\Omega})$. There exists a $\theta \in C_{0}(\bar{\Omega})$ such that $0 \leq \theta \leq 1$ in $\Omega$. For any $v \in \mathcal{O}_{\varepsilon}(\mathcal{S}, \Omega)$ we construct a sequence $v_{n}=v+\theta \| \mathcal{S}_{n}-$ $\mathcal{S} \|_{C(\bar{\Omega})}$. Then $v_{n} \in W(\Omega)$ and $v_{n} \geq \mathcal{S}+\varepsilon H_{\text {mem }}+\left(\mathcal{S}_{n}-\mathcal{S}\right)=\mathcal{S}_{n}+\varepsilon H_{\text {mem }}$ holds for a.e. $[x, y] \in \Omega$, so that $v_{n} \in \mathcal{O}_{e}\left(\mathcal{S}_{n}, \Omega\right)$. Moreover, $\left\|v_{n}-v\right\|_{W(\Omega)}=\left\|\mathcal{S}_{n}-\mathcal{S}\right\|_{C(\bar{\Omega})}\|\theta\|_{W(\Omega)} \rightarrow 0$. Next, let $v_{n} \in \mathcal{O}_{\varepsilon}\left(\mathcal{S}_{n}, \Omega\right)$, with $v_{n} \rightarrow v$ weakly in $W(\Omega)$. Then due to the Rellich theorem, we have $v_{n} \rightarrow v$ strongly in $L_{2}(\Omega)$, since $v_{n} \rightarrow v$ weakly in $H^{1}(\Omega)$ for a.e. $[x, y] \in \Omega$ and $v_{n} \geq \mathcal{S}_{n}+\varepsilon H_{\text {mem }}$ a.e. in $\Omega$. From the Lebesgue theorem, $v \geq \mathcal{S}+\varepsilon H_{\text {mem }}$ follows a.e. in $\Omega$ so that $v \in \mathcal{O}_{\varepsilon}(\mathcal{S}, \Omega)$. Then the proof of Lemma 5 is completed

Lemma 6. For any $S \in U_{a d}^{\mathcal{S}}(\Omega)$ the set $\mathcal{K}_{e}(\mathcal{S}, \Omega) \cap C^{\infty}(\bar{\Omega})$ is dense in $\mathcal{K}_{\varepsilon}(\mathcal{S}, \Omega)$.

Proof. Let $v \in \mathcal{K}_{e}(\mathcal{S}, \Omega)$ be an arbitrary element and let $\mathcal{Z} \in H_{0}^{2}(\Omega)$ be a function such that $\|\mathcal{Z}\|_{H_{0}^{2}(\Omega)}=1$ and $\mathcal{Z}>0$ in $\Omega$. Define the function $v_{(\varepsilon)}$ as $v_{(\varepsilon)}=v+\varepsilon \mathcal{Z}$ for $\varepsilon>0$. Obviously, one has

$$
\left\|v_{\langle\epsilon\rangle}-v\right\|_{V(\Omega)}=\varepsilon\|\mathcal{Z}\|_{H_{0}^{2}(\Omega)}=\varepsilon \quad \text { and } \quad v_{(e)}>v
$$

Since $\mathcal{S} \in U_{\text {ad }}^{S}(\Omega)$ which leads to the assumption $\varepsilon H_{\text {plate }}+\mathcal{S}(\partial \Omega)<0$ we have $v_{\langle\varepsilon\rangle}>$ $\varepsilon H_{\text {plate }}+\mathcal{S}$ in $\Omega$ for any $\varepsilon>0$. From the definition of $H^{2}(\Omega)$ it follows that there exist $v_{(c)} \in C^{\infty}(\bar{\Omega})$ such that $\left\|v_{(c)}-v_{\varepsilon(n)}\right\|_{H^{2}(\Omega)} \rightarrow 0$ for $n \rightarrow \infty$. On the other hand, in view of the embedding theorem of $V(\Omega)$ into $C(\bar{\Omega})$ we may write $v_{\langle\varepsilon\rangle} \rightarrow v_{\langle e\rangle}$ uniformly in $\bar{\Omega}$. Consequently, $v_{\langle\varepsilon\rangle n}>\varepsilon H_{\text {plate }}+\mathcal{S}$ in $\bar{\Omega}$ for $n$ large enough. Therefore one has $v_{\langle e\rangle} \in \mathcal{K}_{\varepsilon}(\mathcal{S}, \Omega) \cap C^{\infty}(\bar{\Omega})$. This prove our lemma

Lemma 7. For any $\mathcal{S} \in U_{\text {ad }}^{\mathcal{S}}(\Omega)$ the set $\mathcal{O}_{\varepsilon}(\mathcal{S}, \Omega) \cap C_{0}^{\infty}(\Omega)$ is dense in $\mathcal{O}_{\varepsilon}(\mathcal{S}, \Omega)$.

Proof. Let $v \in \mathcal{O}_{\varepsilon}(\mathcal{S}, \Omega)$. In view of the definition of the space $W(\Omega)$ (since $\left.\mathcal{O}_{\varepsilon}(S, \Omega) \subset W(\Omega)\right)$ we may write $\left\|\mathbf{o}_{n}-v\right\|_{W(\Omega)} \rightarrow 0$ for $n \rightarrow \infty$ where the sequence $\left\{\mathbf{o}_{n}\right\}_{n \in \mathbb{N}}$ belongs to the space $C_{0}^{\infty}(\Omega)$. Let $v_{n}:=\max \left[\mathbf{o}_{n}, \varepsilon H_{\text {mem }}+\mathcal{S}\right]$ so that

$$
v_{n}=\frac{1}{2}\left[\left(\mathcal{S}+\varepsilon H_{\mathrm{mem}}+\mathbf{o}_{n}\right)+\left|\mathcal{S}+\varepsilon H_{\mathrm{mem}}-\mathbf{o}_{n}\right|\right] .
$$

Then due to that $v \in \mathcal{O}_{\varepsilon}(\mathcal{S}, \Omega)$ and since the map $v \rightarrow|v|$ is continuous [13] we get

$$
\lim _{n \rightarrow \infty} v_{n}=\frac{1}{2}\left[\left(\mathcal{S}+\varepsilon H_{\mathrm{mem}}+v\right)+\left|\mathcal{S}+\varepsilon H_{\mathrm{mem}}-v\right|\right]=\max \left[v, \mathcal{S}+\varepsilon H_{\mathrm{mem}}\right]=v
$$

strongly in $W(\Omega)$. It should be noted that for any $n \in \mathbb{N}$ the function $v_{n}$ has a compact support in $\Omega$ and $v_{n} \in \mathcal{O}_{\varepsilon}(\mathcal{S}, \Omega)$. Taking into account the above assertion, the set $\left\{v \in \mathcal{O}_{\varepsilon}(\mathcal{S}, \Omega) \cap C_{0}(\bar{\Omega})\right\}$ is dense in $\mathcal{O}_{\varepsilon}(\mathcal{S}, \Omega)$ (the function $v$ has a compact support in $\Omega$ ). In the following we consider a domain $\Omega$. such that $\bar{\Omega} \subset \Omega^{*}$. We extend the function 
$v \in \mathcal{O}_{\varepsilon}(S, \Omega) \cap C_{0}(\bar{\Omega})$ by assigning to it the value zero in the outer neighbourhood of $\Omega$. This means that the extension $E v$ of $v$ is defined by

$$
E v(x, y)= \begin{cases}v(x, y) & \text { if }(x, y) \in \Omega \\ 0 & \text { if }(x, y) \in \Omega^{*} \backslash \Omega .\end{cases}
$$

Let us regularize the extension $E v$ using the formula

(M1) $R_{\mathcal{K}} \mathcal{J}(x, y)=A \mathcal{K}^{-1} \int_{\mathbb{R}^{2}} \omega_{\mathcal{K}}([x, y]-[\xi, \eta]) \mathcal{J}(\xi, \eta) d \xi d \eta$

where the mollifier $\omega_{\mathcal{K}}$ is given by

$$
\omega_{\mathcal{K}}([\vartheta, \theta])= \begin{cases}\exp \frac{\| \vartheta, \theta] \|^{2}}{\| \vartheta, \theta] \|^{2}-\kappa^{2}} & \text { for } \| \vartheta, \theta] \mid \leq \kappa \\ 0 & \text { for } \| \vartheta, \theta] \mid>\kappa\end{cases}
$$

and $A, \mathcal{K}$ are positive constants, such that $R_{\mathcal{K}} a=a$ if $a$ is constant. Moreover, for the sequence of mollifiers we have $\omega_{\mathcal{K}_{n}} \in \mathcal{D}\left(\mathbb{R}^{2}\right), \omega_{\mathcal{K}_{n}} \geq 0, \bigcap_{n=1}^{\infty} \operatorname{supp}\left(\omega_{\mathcal{K}_{n}}\right)=\{0\}$ and $\left\{\operatorname{supp}\left(\omega_{\mathcal{K}_{n}}\right)\right\}_{n \in N}$ is a decreasing sequence.

By virtue of (4.10) one has, $E v \in H^{1}\left(R^{2}\right)$. Then we get

$$
\left.\begin{array}{r}
R_{\mathcal{K}_{n}} E v \in \mathcal{D}\left(R^{2}\right) \text { and } \operatorname{supp}\left(R_{\mathcal{K}_{n}} E v\right) \subset \operatorname{supp}(v)+\operatorname{supp}\left(\omega_{\mathcal{K}_{n}}\right) \\
\lim _{n \rightarrow \infty} R_{\mathcal{K}_{n}} E v=E v \text { strongly in } H^{1}\left(R^{2}\right)
\end{array}\right\}
$$

Next, in view of (4.11) one has

$$
\operatorname{supp}\left(\left|R_{\mathcal{K}_{n}} E v\right|\right) \subset E v \quad \text { for } n \text { large enough. }
$$

On the other hand we may write (we recall that $\operatorname{supp}(E v)$ is bounded)

$$
\lim _{n \rightarrow \infty} R_{\mathcal{K}_{n}} E v=E v \quad \text { strongly in } L_{\infty}\left(\mathbb{R}^{2}\right)
$$

We now define the restriction of the function $R_{\mathcal{K}_{n}} E v$ on the domain $\Omega$ and we have $v_{n}=\left.R_{\mathcal{K}_{n}} E v\right|_{\Omega}$, which due to (4.11) - (4.13) gives

$$
v_{n} \in C_{0}^{\infty}(\Omega) \quad \text { and } \quad \lim _{n \rightarrow \infty} v_{n}=v \text { strongly in } W(\Omega) \cap C_{0}(\bar{\Omega}) .
$$

It should by noted that, for any $v \in \mathcal{O}_{e}(\mathcal{S}, \Omega) \cap C_{0}(\bar{\Omega})$ and $\mathcal{S}+\varepsilon H_{\text {mem }} \leq 0$ in a neighbourhood of $\partial \Omega$, there exists a $\varepsilon>0$ such that

$$
\left.\begin{array}{rl}
v & =0 \\
\mathcal{S}+\varepsilon H_{\mathrm{mem}} & <0 \text { on } \Omega_{\mathcal{O}}
\end{array}\right\}
$$

where $\Omega_{\mathcal{O}}=\{[x, y] \in \Omega: \mathrm{d}([x, y], \partial \Omega)<\mathcal{O}\}$ and $\mathrm{d}([x, y], \partial \Omega)$ is the distance from $[x, y]$ to $\partial \Omega$. Then, by taking (4.13) and (4.15), for any $\varepsilon>0$ there exists an $n_{*}=n_{*}(\varepsilon)$ such that for $n \geq n_{*}(\varepsilon)$ one has

$$
\begin{cases}v(x, y)-\varepsilon H_{\text {mem }} \leq v_{n}(x, y) \leq v(x, y)+\varepsilon H_{\text {mem }} & \text { for }[x, y] \in \Omega \backslash \Omega_{\mathcal{O} / 2} \\ v_{n}(x, y)=v(x, y) & \text { for }[x, y] \in \Omega_{\mathcal{O} / 2}\end{cases}
$$


We observe that $\left[\bar{\Omega} \backslash \Omega_{O / 2}\right]$ is a compact subset of $\bar{\Omega}$. Thus, there exists a function $\vartheta$ such that

$$
\vartheta \in C_{0}^{\infty}(\Omega), \quad \vartheta \geq 0 \text { in } \Omega, \quad \vartheta(x, y)=1 \text { for any }[x, y] \in \bar{\Omega} \backslash \Omega_{\mathcal{O} / 2} .
$$

This means that by (4.14), (4.16) and (4.17) we get for the sequence $\left\{\mathcal{Q}_{\langle\varepsilon\rangle_{n}}\right\}_{n \in \mathbb{N}}$ defined by $\mathcal{Q}_{\langle\varepsilon\rangle n}=v_{n}+\varepsilon v$ the relation $\mathcal{Q}_{\langle\varepsilon\rangle n} \in C_{0}^{\infty}(\Omega), \lim _{\varepsilon \rightarrow 0, n \rightarrow \infty} \mathcal{Q}_{\langle\varepsilon\rangle_{n}}=v$ strongly in $W(\Omega)$, for $n \geq n_{*}(\varepsilon), \mathcal{Q}_{(\varepsilon) n}(x, y) \geq v(x, y) \geq \mathcal{S}(x, y)+\varepsilon H_{\text {mem }}$, for any $[x, y] \in \Omega$. Consequently, for every $v \in \mathcal{O}_{e}(S, \Omega) \cap C_{0}(\bar{\Omega})$, there exists a sequence $\left\{v_{k}\right\}_{k \in N}$ such that $v_{k} \in \mathcal{O}_{e}(\mathcal{S}, \Omega) \cap C_{0}^{\infty}(\Omega)$ for any $k$ and $\lim _{k \rightarrow \infty} v_{k}=v$ strongly in $W(\Omega)$. This proves the lemma

In the following we show that $\mathcal{O}_{\varepsilon}(\mathcal{S}, \Omega)$ is the closure of $\mathcal{K}_{\varepsilon}(\mathcal{S}, \Omega)$ in $W(\Omega)$ (every element $v \in \mathcal{O}_{\varepsilon}(\mathcal{S}, \Omega)$ can be approximated by a sequence $\left\{v_{n}\right\}_{n \in \mathbb{N}} \subset \mathcal{K}_{\varepsilon}(\mathcal{S}, \Omega)$ such that $v_{n} \rightarrow v$ strongly in $W(\Omega)$, as $\left.n \rightarrow \infty\right)$.

Lemma 8. For any fixed element $\mathcal{S} \in U_{a d}^{\mathcal{S}}(\Omega)$ one has $\mathcal{O}_{e}(\mathcal{S}, \Omega)$ is the closure of $\mathcal{K}_{\varepsilon}(\mathcal{S}, \Omega)$ in $W(\Omega)$.

Proof. We consider a domain $\Omega_{*}$ such that $\bar{\Omega} \subset \Omega_{*}$. We extend an element $v \in$ $\mathcal{O}_{e}(\mathcal{S}, \Omega) \cap H_{0, p}^{1}(\Omega)$ assuming for its value zero in an outer neighbourhood of $\Omega\left(H_{0, p}^{1}(\Omega)\right.$ is the space of functions, having first derivatives integrable with the power $p>2$ and vanishing on the boundary $\partial \Omega$. Note hat these functions are continuous in $\bar{\Omega}$ ). As well we extend the obstacle function $\mathcal{S}_{\varepsilon, \text { plate }}$ in theirs neighbourhood. In the following we use the continuity of $v$ in $\bar{\Omega}$. Let us regularise the extension $[E v]$ and $\left[E \mathcal{S}_{e, \text { plate }}\right]$ using formula (M1).

For every $n$ we take $\mathcal{K}_{n}$ such that

$$
R_{\mathcal{K}_{n}} E v(x, y)+\frac{1}{n} \geq R_{\mathcal{K}_{n}} E \mathcal{S}_{e, \text { plate }}(x, y)+\frac{1}{n} \geq \mathcal{S}_{e, \text { plate }}(x, y)
$$

in $\Omega$, where $\left[E v, E \mathcal{S}_{\varepsilon, \text { plate }}\right]$ are the above extensions, $\mathcal{S}_{\varepsilon, \text { plate }}=\mathcal{S}+\varepsilon H_{\text {plate }}$ and $\mathcal{S}_{\varepsilon, \text { mem }}=$ $\mathcal{S}+\varepsilon H_{\text {mem }}$. Let us consider in $(\Omega * \backslash \Omega)$ the sequence $\left\{o_{n}\right\}_{n \in N}$ where $o_{n}=R_{\mathcal{K}_{n}} E v+\frac{1}{n}$. On the other hand, for sufficiently large $n$ the functions $R_{\mathcal{K}_{n}} E v$ are equal to zero when $\operatorname{dis}\left([x, y], \partial \Omega_{*}\right) \geq \mathcal{K}_{n}$. Thus we may write

$$
\left\|\mathbf{o}_{n}\right\|_{H^{1}(\Omega . \mid \Omega)} \rightarrow 0 \quad \text { as } n \rightarrow \infty
$$

Let us choose a domain $\mathrm{O}$ with $\overline{\mathcal{O}} \subset \Omega$, and extend the functions $o_{n}$ in $\Omega$ assuming they vanish in $\mathrm{O}$. Then for the extension Eo we may write the estimate

$$
\|E \mathbf{o}\|_{H^{\prime}(\Omega . \mid O)} \leq M\left\|\mathbf{o}_{n}\right\|_{H^{\prime}(\Omega \cdot \mid \Omega)}
$$

Further, assume that $\mathcal{S}_{\varepsilon, \text { plate }}<\vartheta<\left(\varepsilon H_{\text {plate }}+\left(-M_{\min }\right)\right)<0$ and $v>\frac{\vartheta}{2}$ in some neighbourhood $\Omega_{\mathcal{O}}$ of the boundary $\partial \Omega, \mathcal{O}=\Omega \backslash \bar{\Omega}_{0}$. Next, due to estimate (4.20) one has

$$
\left\|E \mathrm{o}_{n}\right\|_{H^{\prime}(\Omega \backslash \mathcal{O})} \leq M_{*}\left\|\mathbf{o}_{n}\right\|_{H^{1}(\Omega \cdot \backslash \Omega)}
$$


Moreover, by virtue of (4.19), the right-hand side of this inequality converges to zero as $n \rightarrow \infty$. From the continuity of $E v$ it follows that

$$
\left\|R_{\mathcal{K}_{n}} E v\right\|_{C(\overline{\Omega \cdot \mid \Omega)}} \rightarrow 0 \quad \text { for } n \rightarrow \infty
$$

But for the extension $E o_{n}$ we may assume that the estimate

$$
\left\|E \mathrm{o}_{n}\right\|_{C(\overline{\Omega \backslash \Omega})} \leq M_{\mathrm{o}}\left\|\mathrm{o}_{n}\right\|_{C(\overline{\Omega \cdot \mid \Omega})}
$$

holds uniformly with respect to $n$. We can deduce from (4.22) that the right-hand side converges to zero for $n \rightarrow \infty$. Thus we have for the extensions the assertions

$$
\left.\begin{array}{l}
E o_{n}(x, y)=R \mathcal{K}_{n} E v(x, y)=\frac{1}{n} \cdot([x, y] \in \partial \Omega) \\
\left\|E o_{n}\right\|_{H^{1}(\Omega / \mathcal{O})} \rightarrow 0 \text { and }\left\|E o_{n}\right\|_{C(\overline{\Omega \backslash \mathcal{O}})} \rightarrow 0 .
\end{array}\right\}
$$

By virtue of (4.18) we have

$$
R_{\mathcal{K}_{n}} E v(x, y)+\frac{1}{n} \geq \mathcal{S}_{\varepsilon, \text { plate }}
$$

for all $[x, y] \in \Omega$. But the right-hand side of (4.24) is bounded from above in $\Omega_{0}$ by a negative constant $\vartheta$, whilst the left-hand side converges to $v$ uniformly with respect to $[x, y]$ in the same neighbourhood (where $v>\frac{\vartheta}{2}$ in $\Omega_{0}$ ). This gives the estimate (due to $(4.23))$

$$
R_{\mathcal{K}_{n}} E v(x, y)+\frac{1}{n}-E o_{n}(x, y) \geq \mathcal{S}_{\varepsilon, \text { plate }}(x, y)>\mathcal{S}_{\varepsilon, \text { mem }}\left(H_{\text {plate }}>H_{\text {mem }}\right)
$$

for all $[x, y] \in \Omega$. Then the sequence $v_{n}=R_{\mathcal{K}_{n}} E v+\frac{1}{n}-E o_{n}$ belongs to the convex set $\mathcal{K}_{e}\left(\mathcal{S}_{n}, \Omega\right)$ and $v_{n} \rightarrow v$ strongly in $H_{0}^{1}(\Omega), v \in \mathcal{O}_{e}(\mathcal{S}, \Omega) \cap H_{0, p}^{1}(\Omega)$. Note that for $v \in \mathcal{O}_{\varepsilon}(\mathcal{S}, \Omega)$ there exists a sequence $v_{n}^{*} \in \mathcal{O}_{\varepsilon}(S, \Omega) \cap H_{0, p}^{1}(\Omega)$ such that $v_{n}^{*} \rightarrow v$ strongly in $H_{0}^{1}(\Omega)$. On the other hand, we may choose a sequence from $\mathcal{K}_{e}(\mathcal{S}, \Omega)$ strongly converging to $v_{n}^{*}$ in $H_{0}^{1}(\Omega)$. Thus the proof of the lemma is complete

Since $\mathcal{L}(e, v)$ is weakly lower semicontinuous on $L_{2}(\Omega)$ we have

$$
\liminf _{n \rightarrow \infty} \mathcal{L}\left(e_{n}, v_{n}\right)=\liminf _{n \rightarrow \infty}\left[\left\|v_{n}-z_{d}\right\|_{L_{2}(\Omega)}^{2} \geq\left\|v-z_{d}\right\|_{L_{2}(\Omega)}^{2}\right.
$$

for $v_{n} \in V(\Omega)$ and $v \in W(\Omega)$ with $v_{n} \rightarrow v$ strongly in $U(\Omega)$. Consequently, condition (E0) is verified. From the above arguments it follows that all the assumptions of Theorems $1-3$ are satisfied. Hence there exists at least one solution of the optimization problems $\left(\mathcal{P}_{e}\right)$ and $\left(\mathcal{P}_{0}\right)$, respectively.

4.1 Approximation by finite elements. Standard Galerkin. We shall propose approximate solutions of the optimization problem for a thin plate by the finite element method. We restrict ourselves to particular domains, namely we suppose that $\Omega$ is a parallelogram. 
Consider a classical quadrilateral mesh $\mathcal{T}_{h}$ of $\Omega$, i.e. $\mathcal{T}_{h}$ is a finite set of parallelograms $G_{i}$, by means of two systems of equidistant straight lines parallel with the sides $\Omega$. Then we may write

$$
\begin{aligned}
& G_{j} \subset \bar{\Omega} \text { for any } G_{j} \in \mathcal{T}_{h} \\
& \cup_{G \in \mathcal{T}_{h}} G=\bar{\Omega} \\
& \dot{G}_{1} \cap \dot{G}_{2}=\emptyset \text { for any } G_{1}, G_{2} \in \mathcal{T}_{h} \text { such that } G_{1} \neq G_{2}
\end{aligned}
$$

where $\dot{G}_{1}$ denotes the interior of $G_{1}$. Moreover, for any $G_{1}, G_{2} \in \mathcal{T}_{h}$ with $G_{1} \neq G_{2}$, exactly one of the conditions

$1^{0} G_{1} \cap G_{2}=\emptyset$

$2^{0} G_{1}$ and $G_{2}$ have only a whole common vertex

$3^{0} G_{1}$ and $G_{2}$ have only a whole common edge

must hold. As usual $h$ will be the length of the largest edge of the parallelograms in the quadrilateral mesh. Furthermore, we assume that $\mathcal{T}_{h}$ is consistent with the partition of the boundary $\partial \Omega$. Thus we may write $\partial \Omega=\bigcup_{j=1}^{n(h)} \overline{A_{j-1} A_{j}}$ where $A_{j}$ is the vertex of $G$ in $\mathcal{T}_{h}$.

In what follows, we shall consider only families $\left\{\mathcal{T}_{h}\right\}(h \rightarrow 0)$ of such partitions, which refine the "original" partition $\mathcal{T}_{h}$. We shall say that a family $\left\{\mathcal{T}_{h}\right\}$ is regular, if there exists a constant $c>0$ such that $\frac{h}{\rho} \leq$ const for any $G_{i} \in \bigcup_{h} \mathcal{T}_{h}$, where $\rho$ denotes the diameter of the maximal circle contained in $G$. We suppose that the condition

$$
\mathcal{T}_{h_{1}} \subset \mathcal{T}_{h_{2}} \quad \text { if } h_{1}>h_{2}
$$

is satisfied.

We introduce the spaces $Q_{k}(G)$ of bilinear (if $k=1$ ) or bicubic (if $k=3$ ) polynomials defined on the quadrilateral (see, e.g., $[9,12])$. We denote

$$
\begin{aligned}
\mathcal{W}_{h} & =\{A \in \bar{\Omega}: A \text { is a vertex } G \in \mathcal{T}\} \\
\dot{\mathcal{W}}_{h} & =\left\{A \in \mathcal{W}_{h}: A \notin \partial \Omega\right\} \\
\Gamma_{h} & =\left\{A \notin \dot{\mathcal{W}}_{h}: A \in \partial \Omega\right\} .
\end{aligned}
$$

The spaces $V(\Omega), W(\Omega)$ and $U_{a d}(\Omega)$ are approximated by the families of subspaces $\left\{V_{h_{n}}(\Omega)\right\}_{n \in \mathbb{N}},\left\{W_{h_{n}}(\Omega)\right\}_{n \in N}$ and $\left\{U_{a d}^{h_{n}}(\Omega)\right\}_{n \in N}$, respectively, where

$$
\begin{aligned}
V_{h}(\Omega) & =\left\{v \in V(\Omega): v / G \in Q_{3}(G) \text { for any } G \in \mathcal{T}_{h}\right\} \\
W_{h}(\Omega) & =\left\{v \in W(\Omega): v / G \in Q_{1}(G) \text { for any } G \in \mathcal{T}_{h}\right\} \\
U_{a d}^{E, h}(\Omega) & =\left\{v \in U_{a d}^{E}(\Omega): E / G \in Q_{0}(G) \text { for any } G \in \mathcal{T}_{h}\right\} \\
U_{a d}^{q, h}(\Omega) & =\left\{v \in U_{a d}^{q}(\Omega): q / G \in Q_{1}(G) \text { for any } G \in \mathcal{T}_{h}\right\} \\
U_{a d}^{\mathcal{S}, h}(\Omega) & =\left\{v \in U_{a d}^{\mathcal{S}}(\Omega): \mathcal{S} / G \in Q_{3}(G) \text { for any } G \in \mathcal{T}_{h}\right\} .
\end{aligned}
$$

Clearly, such defined subspaces $V_{h}(\Omega), W_{h}(\Omega), U_{a d}^{E, h}(\Omega), U_{a d}^{q, h}(\Omega), U_{a d}^{\mathcal{S}, h}(\Omega)$ are finitedimensional (see [12]). It is then quite natural to approximate $\mathcal{K}_{\varepsilon}(\mathcal{S}, \Omega), \mathcal{O}_{\varepsilon}(\mathcal{S}, \Omega)$ by

$$
\begin{aligned}
& \mathcal{K}_{e, h}\left(\mathcal{S}_{h}, \Omega\right)=\left\{v_{h} \in V_{h}(\Omega): v_{h}\left(A_{i}^{h}\right) \geq \mathcal{S}_{h}\left(A_{i}^{h}\right)+\varepsilon H_{\text {plate }} \forall A_{i}^{h} \in \dot{\mathcal{W}}_{h}, \text { i.e. } A_{i}^{h} \in \Omega\right\} \\
& \mathcal{O}_{\varepsilon, h}\left(\mathcal{S}_{h}, \Omega\right)=\left\{v_{h} \in W_{h}(\Omega): v_{h}\left(A_{i}^{h}\right) \geq \mathcal{S}_{h}\left(A_{i}^{h}\right)+\varepsilon H_{\text {mem }} \forall A_{i}^{h} \in \dot{\mathcal{W}}_{h}, \text { i.e. } A_{i}^{h} \in \Omega\right\},
\end{aligned}
$$


respectively.

Let us consider the following discrete variants of $a(E, v, z), b(E, v, z),\langle\mathcal{J}(q), v\rangle_{W(\Omega)}$ for any $e_{h} \in U_{a d}^{h}(\Omega)$ :

$$
\begin{aligned}
& a\left(E_{h}, v_{h}, z_{h}\right) \\
& =\int_{\Omega}\left[\mathcal{N}_{x x}\left(v_{h}\right), \mathcal{N}_{y y}\left(v_{h}\right), \mathcal{N}_{x y}\left(v_{h}\right)\right]\left[Q_{\mathcal{A}}\left(E_{h}\right)\right]\left[\mathcal{N}_{x x}\left(z_{h}\right), \mathcal{N}_{y y}\left(z_{h}\right), \mathcal{N}_{x y}\left(z_{h}\right)\right]^{T} d \Omega
\end{aligned}
$$

for any $v_{h}, z_{h} \in V_{h}(\Omega)$,

$$
\left\langle\mathcal{J}(q), v_{h}\right\rangle_{W(\Omega)}=\sum_{a=1}^{M} F_{a} v_{h}\left(x_{a}, y_{a}\right)+\int_{\Omega} q v_{h} d \Omega
$$

for any $v_{h} \in V_{h}(\Omega)$, and

$$
b\left(E_{h}, v_{h}, z_{h}\right)=\int_{\Omega} Q_{B}\left(E_{h}\right)\left(\mathcal{N}_{x}\left(v_{h}\right) \mathcal{N}_{x}\left(z_{h}\right)+\mathcal{N}_{y}\left(v_{h}\right) \mathcal{N}_{y}\left(z_{h}\right)\right) d \Omega
$$

for any $v_{h}, z_{h} \in W_{h}(\Omega)$. On the other hand, the linear operators

$$
\left.\begin{array}{l}
\mathcal{A}_{h}\left(E_{h}\right): V_{h}(\Omega) \rightarrow V_{h}^{*}(\Omega) \\
\mathcal{B}_{h}\left(E_{h}\right): W_{h}(\Omega) \rightarrow W_{h}^{*}(\Omega)
\end{array}\right\}
$$

define the discrete bilinear forms

$$
\left.\begin{array}{rl}
\left\langle\mathcal{A}_{h}\left(E_{h}\right) v_{h}, z_{h}\right\rangle_{v_{h}(\Omega)} & =a_{h}\left(E_{h}, v_{h}, z_{h}\right) \\
\left\langle\mathcal{B}_{h}\left(E_{h}\right) v_{h}, z_{h}\right\rangle_{W_{h}(\Omega)} & =b_{h}\left(E_{h}, v_{h}, z_{h}\right)
\end{array}\right\}
$$

respectively. In the following we assume that

$$
\begin{aligned}
\left\langle\mathcal{A}_{h}\left(E_{h}\right) v_{h}, z_{h}\right\rangle_{V_{h}(\Omega)} & : \equiv\left\langle\mathcal{A}\left(E_{h}\right) v_{h}, z_{h}\right\rangle_{V(\Omega)} \\
\left\langle\mathcal{J}_{h}\left(q_{h}\right), v_{h}\right\rangle_{W_{h}(\Omega)} & : \equiv\left\langle\mathcal{J}\left(q_{h}\right), v_{h}\right\rangle_{W(\Omega)} \\
\left\langle\mathcal{B}_{h}\left(E_{h}\right) v_{h}, z_{h}\right\rangle_{W_{h}(\Omega)} & : \equiv\left\langle\mathcal{B}\left(E_{h}\right) v_{h}, z_{h}\right\rangle_{W(\Omega)} \\
\mathcal{L}_{h}\left(e_{h}, v_{h}\right) & :=\mathcal{L}\left(e_{h}, v_{h}\right)
\end{aligned}
$$

This means that no numerical integration is used in the problem. The approximations of $\left(\mathcal{P}_{h \varepsilon}\right)$ and $\left(\mathcal{P}_{h 0}\right)$ are obvious now.

Due to the above made choice assumptions $(H 1)_{\mathcal{A}_{h}} / 1^{0}, 4^{0}$ and $(H 1)_{B_{h}} / 1^{0}$ are satisfied for our problem. Assumptions $(H 1)_{B_{h}} / 5^{0}, 6^{0}$ are satisfied too, as $B_{h} \equiv 0$ for $h \in(0,1)$. Let us check assumptions $(H 1)_{\mathcal{A}_{h}} / 2^{0}, 3^{0}$ and $(H 1)_{B_{h}} / 2^{0}, 3^{0}$. If $E_{h_{n}} \rightarrow E$ in $R$ and $q_{h_{n}} \rightarrow q$ uniformly in $\bar{\Omega}, \mathcal{S}_{h_{n}} \rightarrow \mathcal{S}$ strongly in $H^{2}(\Omega), v_{h_{n}} \rightarrow v$ weakly in $V(\Omega)$, 
$z_{h_{n}} \rightarrow z$ strongly in $V(\Omega)$, then

$$
\begin{aligned}
\int_{\Omega}\left[\mathcal{N}_{x x}\left(v_{h_{n}}\right), \mathcal{N}_{y y}\left(v_{h_{n}}\right), \mathcal{N}_{x y}\left(v_{h_{n}}\right)\right] \\
\quad \times\left[Q_{\mathcal{A}}\left(E_{h_{n}}\right)\right]\left[\mathcal{N}_{x x}\left(z_{h_{n}}\right), \mathcal{N}_{y y}\left(z_{h_{n}}\right), \mathcal{N}_{x y}\left(z_{h_{n}}\right)\right]^{T} d \Omega \\
=\int_{\Omega}\left[\mathcal{N}_{x x}\left(v_{h_{n}}\right), \mathcal{N}_{y y}\left(v_{h_{n}}\right), \mathcal{N}_{x y}\left(v_{h_{n}}\right)\right] \\
\quad \times\left[Q_{A}\left(E_{h_{n}}-E\right)\right]\left[\mathcal{N}_{x x}\left(z_{h_{n}}\right), \mathcal{N}_{y y}\left(z_{h_{n}}\right), \mathcal{N}_{x y}\left(z_{h_{n}}\right)\right]^{T} d \Omega \\
\quad+\int_{\Omega}\left[\mathcal{N}_{x x}\left(v_{h_{n}}\right), \mathcal{N}_{y y}\left(v_{h_{n}}\right), \mathcal{N}_{x y}\left(v_{h_{n}}\right)\right] \\
\quad \times\left[Q_{\mathcal{A}}(E)\right]\left[\mathcal{N}_{x x}\left(z_{h_{n}}\right), \mathcal{N}_{y y}\left(z_{h_{n}}\right), \mathcal{N}_{x y}\left(z_{h_{n}}\right)\right]^{T} d \Omega \\
\rightarrow \int_{\Omega}\left[\mathcal{N}_{x x}(v), \mathcal{N}_{y y}(v), \mathcal{N}_{x y}(v)\right]\left[Q_{\mathcal{A}}(E)\right]\left[\mathcal{N}_{x x}(z), \mathcal{N}_{y y}(z), \mathcal{N}_{x y}(z)\right]^{T} d \Omega
\end{aligned}
$$

and

$$
\begin{aligned}
\liminf _{h_{n} \rightarrow 0} & \int_{\Omega}\left[\mathcal{N}_{x x}\left(v_{h_{n}}\right), \mathcal{N}_{y y}\left(v_{h_{n}}\right), \mathcal{N}_{x y}\left(v_{h_{n}}\right)\right] \\
& \times\left[Q_{\mathcal{A}}\left(E_{h_{n}}\right)\right]\left[\mathcal{N}_{x x}\left(v_{h_{n}}\right), \mathcal{N}_{y y}\left(v_{h_{n}}\right), \mathcal{N}_{x y}\left(v_{h_{n}}\right)\right]^{T} d \Omega \\
\geq & \liminf _{h_{n} \rightarrow 0} \int_{\Omega}\left[\mathcal{N}_{x x}\left(v_{h_{n}}\right), \mathcal{N}_{y y}\left(v_{h_{n}}\right), \mathcal{N}_{x y}\left(v_{h_{n}}\right)\right] \\
& \times\left[Q_{\mathcal{A}}(E)\right]\left[\mathcal{N}_{x x}\left(v_{h_{n}}\right), \mathcal{N}_{y y}\left(v_{h_{n}}\right), \mathcal{N}_{x y}\left(v_{h_{n}}\right)\right]^{T} d \Omega \\
& +\lim _{h_{n} \rightarrow 0} \int_{\Omega}\left[\mathcal{N}_{x x}\left(v_{h_{n}}\right), \mathcal{N}_{y y}\left(v_{h_{n}}\right), \mathcal{N}_{x y}\left(v_{h_{n}}\right)\right] \\
& \times\left[Q_{\mathcal{A}}\left(E_{h_{n}}-E\right)\right]\left[\mathcal{N}_{x x}\left(v_{h_{n}}\right), \mathcal{N}_{y y}\left(v_{h_{n}}\right), \mathcal{N}_{x y}\left(v_{h_{n}}\right)\right]^{T} d \Omega \\
\geq & \int_{\Omega}\left[\mathcal{N}_{x x}(v), \mathcal{N}_{y y}(v), \mathcal{N}_{x y}(v)\right]\left[Q_{\mathcal{A}}(E)\right]\left[\mathcal{N}_{x x}(v), \mathcal{N}_{y y}(v), \mathcal{N}_{x y}(v)\right]^{T} d \Omega .
\end{aligned}
$$

In fact, as the form $a(E, \cdot, \cdot)$ is elliptic on $V(\Omega)$ for any $E \in U_{a d}^{E}(\Omega)$, therefore it is weakly lower semicontinuous. Consequently, conditions $(H 1)_{A_{A}} / 2^{0}, 3^{0}$ are verified. Similarly, we can verify conditions $(H 1)_{B_{h}} / 2^{0}, 3^{0}$. Next, the Arzelá-Ascoli theorem and the definition of $U_{a d}(\Omega)$ yield assumption $(H 2)_{h} / 1^{0}$.

The crucial point is to prove assumptions $(H 2)_{h} / 2^{0}$ and $\left((L 0)_{A_{h}}\right),\left((L 0)_{B_{h}}\right)$ : Let $q \in U_{a d}^{q}(\Omega)$ and $I_{h} q \in U^{q, h}(\Omega)$ be the Lagrange linear interpolate of $q$ over $\mathcal{T}_{h}$. Since $q \in W_{\infty}^{1}(\Omega)$, the interpolation theory yields

$$
\left\|q-I_{h} q\right\|_{L_{\infty}(\Omega)} \leq \mathrm{const} \cdot h\|q\|_{W_{\infty}^{1}(\Omega)}
$$

Obviously, $c_{1 q} \leq I_{h} q \leq c_{2 q}$ everywhere in $\bar{\Omega}$. Finally, we have for $\overline{P_{i} P_{i+1}}$ parallel with 
the $x$-axis (or $y$-axis)

$$
\left.\begin{array}{l}
\left|\frac{\partial I_{h} q}{\partial x}\right|=\frac{1}{h_{x}}\left|q\left(P_{i+1}\right)-q\left(P_{i}\right)\right| \leq \frac{1}{h_{x}} \int_{P_{i}}^{P_{i+1}}\left|\frac{\partial q}{\partial x}\right| d x \leq M_{\langle x\rangle} \\
\left|\frac{\partial I_{h} q}{\partial y}\right|=\frac{1}{h_{y}}\left|q\left(P_{i+1}\right)-q\left(P_{i}\right)\right| \leq \frac{1}{h_{y}} \int_{P_{i}}^{P_{i+1}}\left|\frac{\partial q}{\partial y}\right| d y \leq M_{(y)} .
\end{array}\right\}
$$

Consider the parallelogram and use the skew coordinates $\left[\xi_{1}, \eta_{2}\right]$ via the affine mapping

$$
[x, y]=\mathcal{P}([\xi, \eta]): x=\xi+\eta \cos \alpha \text { and } y=\eta \sin \alpha
$$

which maps a rectangle $K$ * onto $K$. Let $v \in Q_{1}(K)$. Then $v \circ \mathcal{P}=\widehat{v} \in Q_{1}\left(K_{*}\right)$. Let $\Omega=\mathcal{P}\left(\Omega_{*}\right), \Omega_{*}=\left(0, L_{x}\right) \times\left(0, L_{y}\right), h_{x}=\frac{L_{x}}{m}$ and $h_{y}=\frac{L_{y}}{n}$. Denote by $F_{i j}$ the grid points with coordinates $\xi=i h_{x}$ and $\eta=j h_{y}{ }^{m}(i=1,2, \ldots, m$ and $j=1,2, \ldots, n)$,

$$
\begin{aligned}
& K_{i j}^{*}=\left[(i-1) h_{x}, i h_{x}\right] \times\left[(j-1) h_{y}, j h_{y}\right], \quad K_{i j}=\mathcal{P}\left(K_{i j}^{*}\right) \\
& H_{i j}^{*}=\left(\left(i-\frac{1}{2}\right) h_{x},\left(i+\frac{1}{2}\right) h_{x}\right) \times\left(\left(j-\frac{1}{2}\right) h_{y},\left(j+\frac{1}{2}\right) h_{y}\right) \cap \Omega_{*}, \quad H_{i j}=\mathcal{P}\left(H_{i j}^{*}\right) .
\end{aligned}
$$

This means that $H_{i j}$ is a "neighbourhood" of the point $\mathcal{P}\left(F_{i j}\right)$. Let us set

$$
I_{h} q\left(\mathcal{P}\left(F_{i j}\right)\right)=\frac{1}{\left.\operatorname{mes} H_{i j}\right)} \int_{H_{i j}} I_{h} q(x, y) d \Omega \quad(0 \leq i \leq m ; 0 \leq j \leq n) .
$$

We may write

$$
\int_{K_{i j}} I_{h} q d \Omega=\frac{1}{4} \operatorname{mes} K_{i j} \sum_{k=1}^{4} I_{h} q\left(F_{i j}^{K}\right)
$$

where $F_{i j}^{K}$ are vertices of the parallelogram $K_{i j}$.

Let $\mathcal{S}_{i j}$ denote the union of all parallelograms $K_{i j}$, which are adjacent to the node $\mathcal{P}\left(F_{i j}\right)$. Then we have

$$
\begin{aligned}
\int_{\Omega} I_{h} q d \Omega & =\sum_{i=1}^{m} \sum_{j=1}^{n} \int_{K_{i j}} I_{h} q d \Omega \\
& =\sum_{i=1}^{m} \sum_{j=1}^{n} \frac{1}{4} \operatorname{mes} K_{i j} \sum_{k=1}^{4} I_{h} q\left(\mathcal{P}\left(F_{i j}^{K}\right)\right) \\
& =\sum_{i=0}^{m} \sum_{j=0}^{n} I_{h} q\left(\mathcal{P}\left(F_{i j}^{K}\right)\right) \frac{1}{4} \operatorname{mes} S_{i j} \\
& =\sum_{i=0}^{m} \sum_{j=0}^{n} \frac{\operatorname{mes} S_{i j}}{4 \operatorname{mes} H_{i j}} \int_{H_{i j}} q d \Omega \\
& =\int_{\Omega} q d \Omega
\end{aligned}
$$


since mes $\mathcal{S}_{i j}=4$ mes $H_{i j}, \cup_{i, j} \bar{H}_{i j}=\Omega$. Let $u_{e}(e) \in V(\Omega)$ be the solution of (1.3) (with respect to (4.2) - (4.4)) and $u_{\varepsilon h}(e) \in V_{h}(\Omega)$ be the solution of (2.1) (with respect to (4.26) - (4.28)). Regarding the regularity of the state function $u_{\varepsilon}(e) \in \mathcal{K}(\mathcal{S}, \Omega)$ it is shown in [4] that $q \in L_{p}(\Omega)$ with $p>2$ implies $u_{\varepsilon} \in W_{p, l o c}^{3}(\Omega)$. Taking into account (4.31), a standard estimate gives

$$
\left\|u_{\varepsilon}(e)-u_{\varepsilon h}\left(I_{h} e\right)\right\|_{H^{2}(\Omega)} \leq M(\varepsilon) h\left\|_{\varepsilon}(e)\right\|_{H^{3}(\Omega)} \leq M_{\bullet}(\varepsilon) h .
$$

Therefore, as $u_{e}(e) \in \mathcal{K}_{e}(\mathcal{S}, \Omega)$ and $u_{e h}\left(I_{h} e\right) \in \mathcal{K}_{\varepsilon, h}\left(\mathcal{S}_{h}, \Omega\right)$ for $h$ sufficiently small (for every $\left.\frac{1}{2} \alpha_{B} \geq \varepsilon>h\right)$. This gives the verification of assumption $(H 2)_{h} / 2^{0}$.

Lemma 9. For any $\mathcal{S} \in U_{\text {ad }}^{\mathcal{S}}(\Omega)$ there exists a sequence $\left\{\mathcal{S}_{h_{n}}\right\}_{n \in \mathbb{N}}$ with $h_{n} \rightarrow 0^{+}$ such that $\mathcal{S}_{h_{n}} \in U_{a d}^{\mathcal{S}, h}(\Omega)$ and

$$
\lim _{h_{n} \rightarrow 0^{+}}\left\|\mathcal{S}_{h_{n}}-\mathcal{S}\right\|_{C(\bar{\Omega})}=0 \text {. }
$$

Proof. Consider the parallelogram $\Omega$ and use the skew coordinates $[\xi, \eta]$ via mapping (4.32). We have $\Omega=\mathcal{P}\left(\Omega_{*}\right), \Omega_{*}=\left(0, L_{x}\right) \times\left(0, L_{y}\right) ; h_{x}=\frac{L_{z}}{m}$ and $h_{y}=\frac{L_{x}}{n}$. In the following we denote by $F_{i j}$ the grid points with coordinates $\xi=i h_{x}$ and $\eta=j h_{y}(i=0,1,2, \ldots, m ; j=0,1,2, \ldots, n)$ :

$$
\begin{aligned}
& \mathcal{Q}_{i j}^{*}=\left[(i-1) h_{x}, i h_{x}\right] \times\left[(j-1) h_{y}, j h_{y}\right], \mathcal{Q}_{i j}=\mathcal{P}\left(\mathcal{Q}_{i j}^{*}\right) \\
& \mathcal{O}_{i j}^{*}=\left(\left(i-\frac{1}{2}\right) h_{x},\left(i+\frac{1}{2}\right) h_{x}\right) \times\left(\left(j-\frac{1}{2}\right) h_{y},\left(j+\frac{1}{2}\right) h_{y}\right) \cap \Omega_{*}, \mathcal{O}_{i j}=\mathcal{P}\left(\mathcal{O}_{i j}^{*}\right) .
\end{aligned}
$$

This means $\mathcal{O}_{i j}$ is a "neighbourhood" of the point $\mathcal{P}\left(F_{i j}\right)$. Let us set

$$
\mathcal{S}_{h}\left(\mathcal{P}\left(F_{i j}\right)\right)=\frac{1}{\operatorname{mes} \mathcal{O}_{i j}} \int_{\mathcal{O}_{i j}} \mathcal{S}(x, y) d x d y \quad(0 \leq i \leq m ; 0 \leq j \leq n) .
$$

Next, we shall show that interpolating the nodal values $(4.34)$ by functions from $Q_{1}\left(\mathcal{Q}_{i j}\right)$, we obtain $\mathcal{S}_{h} \in U_{a d}^{\mathcal{S}, h}(\Omega)$. We may write

$$
\int_{\mathcal{Q}_{i j}} \mathcal{S}_{h} d x d y=\frac{1}{4} \operatorname{mes} \mathcal{Q}_{i j} \sum_{k=1}^{4} \mathcal{S}_{h}\left(F_{i j}^{k}\right)
$$

where $F_{i j}^{k}$ are vertices of the parallelogram $\mathcal{Q}_{i j}$. Let $\mathcal{S}_{i j}$ denote the union of all parallelograms $\mathcal{Q}_{i j}$, which are adjacent to the node $\mathcal{P}\left(F_{i j}\right)$. Then we have

$$
\begin{aligned}
\int_{\Omega} \mathcal{S}_{h} d x d y & =\sum_{i=1}^{m} \sum_{j=1}^{n} \int_{\mathcal{Q}_{i j}} \mathcal{S}_{h} d x d y \\
& =\sum_{i=1}^{m} \sum_{j=1}^{n} \frac{1}{4} \operatorname{mes} \mathcal{Q}_{i j} \sum_{k=1}^{4} S_{h}\left(\mathcal{P}\left(F_{i j}^{k}\right)\right) \\
& =\sum_{i=0}^{m} \sum_{j=0}^{n} \mathcal{S}_{h}\left(\mathcal{P}\left(F_{i j}^{k}\right)\right) \frac{1}{4} \operatorname{mes} \mathcal{S}_{i j} \\
& =\sum_{i=0}^{m} \sum_{j=0}^{n} \frac{\operatorname{mes} \mathcal{S}_{i j}}{4 \operatorname{mes} \mathcal{O}_{i j}} \int_{\mathcal{O}_{i j}} \mathcal{S} d x d y \\
& =\int_{\Omega} \mathcal{S} d x d y \\
& =M_{\mathcal{S}}
\end{aligned}
$$


since mes $S_{i j}=4 \operatorname{mes} \mathcal{O}_{i j}, \cup_{i, j} \overline{\mathcal{O}}_{i j}=\Omega$.

We now introduce the functions $S_{*}=S \circ \mathcal{P}$ and $\mathcal{S}_{*(h)}=S_{h} \circ \mathcal{P}$. Then (4.34) can be transformed into the formula

$$
\mathcal{S}_{*\langle h\rangle}\left(F_{i j}\right)=\frac{1}{\operatorname{mes} O_{i j}^{*}} \int_{O_{i j}^{*}} \mathcal{S}_{*} d \xi d \eta
$$

Moreover, as far as we identify the system $[\xi, \eta]$ with a skew coordinate system, parallel with the edges of $\Omega$, we easily verify that

$$
\frac{\partial \mathcal{S}}{\partial \xi}=\frac{\partial \mathcal{S}_{*}}{\partial \xi}, \quad \frac{\partial \mathcal{S}}{\partial \eta}=\frac{\partial \mathcal{S}_{*}}{\partial \eta}, \quad \frac{\partial \mathcal{S}_{h}}{\partial \xi}=\frac{\partial \mathcal{S}_{*\langle h\rangle}}{\partial \xi}, \quad \frac{\partial \mathcal{S}_{h}}{\partial \eta}=\frac{\partial \mathcal{S}_{*\langle h\rangle}}{\partial \eta}
$$

holds at the corresponding points. Let us extend $\mathcal{S}_{*}$ onto a rectangle

$$
\left(-\frac{1}{2} h_{x}, L_{x}+\frac{1}{2} h_{x}\right) \times\left(-\frac{1}{2} h_{y}, L_{y}+\frac{1}{2} h_{y}\right)
$$

so that the extension $S_{0}=S_{*}$ in $\Omega_{*}$ and $S_{0}$ is symmetric with respect to the sides, namely $S_{0}\left(L_{x}+a, \eta\right)=S_{0}\left(L_{x}-a, \eta\right)$ for any $\eta \in\left(-\frac{1}{2} h_{y}, L_{y}+\frac{1}{2} h_{y}\right)$ and any $a \in\left(0, \frac{1}{2} h_{x}\right)$, and similarly along the other sides of $\partial \Omega$. This means that we may write

$$
\mathcal{S}_{*\langle h\rangle}\left(F_{i j}\right)=\frac{1}{h_{x} h_{y}} \int_{\mathcal{R}_{i j\langle 0\rangle}} \mathcal{S}_{0} d \xi d \eta \quad(0 \leq i \leq \dot{m} ; 0 \leq j \leq n)
$$

instead of (4.35) where $\mathcal{R}_{i j\langle 0\rangle}$ denotes the (complete) rectangle with the center $F_{i j}$ and the lengths of sides $h_{x}$ and $h_{y}$. We have

$$
\begin{aligned}
\frac{1}{h_{x}}\left|\mathcal{S}_{*(h)}\left(F_{i+1, j}\right)-\mathcal{S}_{*(h)}\left(F_{i, j}\right)\right| \\
\quad=\frac{1}{h_{x}^{2} h_{y}}\left|\int_{\mathcal{R}_{i+1, j,(0)}} \mathcal{S}_{0} d \xi d \eta-\int_{\mathcal{R}_{i j\langle 0\rangle}} \mathcal{S}_{0} d \xi d \eta\right| \\
\quad=\frac{1}{h_{x} h_{y}}\left|\int_{\mathcal{R}_{i j(0)}} \frac{1}{h_{x}}\left[\mathcal{S}_{0}\left(\xi+h_{x}, \eta\right)-\mathcal{S}_{0}(\xi, \eta)\right] d \xi d \eta\right| \\
\leq \frac{1}{h_{x} h_{y}} M_{0} \operatorname{mes} R_{i j(0)} \\
=M_{0}
\end{aligned}
$$

making use of the fact that $\left|\frac{\partial \mathcal{S}_{0}}{\partial \xi}\right| \leq M_{0}$ holds almost everywhere. Due to the fact that $\mathcal{S}_{*(h)} \in Q_{1}\left(\mathcal{Q}_{i j}^{*}\right)$ in $\mathcal{Q}_{i j}^{*}$, the derivative $\frac{\partial \mathcal{S}_{\cdot(h)}}{\partial \xi}$ attains its maximum at the boundary $\partial \mathcal{Q}_{i j}^{*}$. Then by virtue of (4.37), we obtain the estimate $\left|\frac{\partial \mathcal{S}_{\mathrm{A}}}{\partial \xi}\right| \leq M_{\mathrm{o}}$ for any $[x, y] \in \Omega$. On the other hand, the upper bound $M_{0}^{*}$ for $\frac{\partial S_{\mathrm{h}}}{\partial \eta}$ can be derived in a parallel way. Observe that the maximum $\mathcal{S}_{*(h)}$ in $\mathcal{Q}_{i j}^{*}$ in attained at some vertex of $\mathcal{Q}_{i j}^{*}$. Thus due to (4.35) we easily verify that $-M_{\max } \leq \mathcal{S}_{h}(x, y) \leq-M_{\min }$ for any $[x, y] \in \bar{\Omega}$. This means that $\mathcal{S}_{h} \in U_{\mathrm{ad}}^{\mathcal{S}, h}(\Omega)$. 
It remains to prove the convergence (4.33). We consider an arbitrary point $[x, y] \in \bar{\Omega}$ and write for $[\xi, \eta] \in \mathcal{P}^{-1}(x, y) \in \mathcal{Q}_{i j}^{*}$

$$
\left|\mathcal{S}_{h}(x, y)-\mathcal{S}(x, y)\right|=\left|\sum_{k=1}^{4} \mathcal{S}_{h(*)}\left(F_{i j}^{k}\right) \mathrm{o}_{k}(\xi, \eta)-\sum_{k=1}^{4} \mathcal{S}(\xi, \eta) \mathrm{o}_{k}(\xi, \eta)\right|
$$

where $\mathbf{o}_{k}$ are the shape functions of $Q_{1}\left(\mathcal{Q}_{i j}^{*}\right)$ (i.e. $\mathbf{o}_{k}\left(F_{i j}^{m}\right)=\delta_{k m}$ holds at the vertices). By virtue of (4.35), we obtain

$$
\begin{aligned}
\left|\mathcal{S}_{h}(x, y)-\mathcal{S}(x, y)\right| & \\
\leq & \sum_{k=1}^{4}\left|\mathcal{S}_{h\langle *\rangle}\left(F_{i j}^{k}\right)-\mathcal{S}_{*}(\xi, \eta) o_{k}(\xi, \eta)\right| \\
= & \sum_{k=1}^{4} \mid \frac{1}{h_{x} h_{y}} \iint_{\mathcal{R}_{i j(0)}^{k}} \mathcal{S}_{0}\left(o_{x}, o_{y}\right) d o_{x} d o_{y} \\
& -\frac{1}{h_{x}, h_{y}} \iint_{\mathcal{R}_{i j(0)}^{k}} \mathcal{S}_{*}(\xi, \eta) d o_{x} d o_{y} \mid o_{k}(\xi, \eta) \\
\leq & \sum_{k=1}^{4} \frac{1}{h_{x} h_{y}} \iint_{\mathcal{R}_{i j\langle 0\rangle}^{k}}\left|\mathcal{S}_{0}\left(o_{x}, o_{y}\right)-\mathcal{S}_{*}(\xi, \eta)\right| d o_{x} d o_{y}
\end{aligned}
$$

where $\mathcal{R}_{i j\langle 0\rangle}^{k}$ denotes the rectangle with the center at $F_{i j}^{k}$, mes $\mathcal{R}_{i j\langle 0\rangle}^{k}=h_{x} h_{y}$. On the other hand, we have

$$
\begin{aligned}
\left|\mathcal{S}_{0}\left(o_{x}, o_{y}\right)-\mathcal{S}_{*}(\xi, \eta)\right| & =\left|\mathcal{S}_{0}\left(o_{x}, o_{y}\right)-\mathcal{S}_{0}(\xi, \eta)\right| \\
& \leq\left|\mathcal{S}_{0}\left(o_{x}, o_{y}\right)-\mathcal{S}_{0}\left(\xi, o_{y}\right)\right|+\left|\mathcal{S}_{0}\left(\xi, o_{y}\right)-\mathcal{S}_{0}(\xi, \eta)\right| \\
& \leq \frac{3}{2}\left(h_{x} M_{o}+h_{y} M_{o}^{*}\right) .
\end{aligned}
$$

Finally, by virtue of (4.39) and (4.38) one has

$$
\left|\mathcal{S}_{h}(x, y)-\mathcal{S}(x, y)\right| \leq 12 h \max \left[M_{\mathrm{o}}, M_{\mathrm{o}}^{*}\right]
$$

which gives (4.33)

Let us verify conditions $(L 0)_{\mathcal{A}_{h}}$ and $(L 0)_{\mathcal{B}_{h}}$.

Lemma 10. For every fixed $v \in \Lambda_{\mathcal{K}(S, \Omega)}=\mathcal{K}_{\varepsilon}(\mathcal{S}, \Omega) \cap C^{\infty}(\bar{\Omega})$ there exists a sequence $\left\{v_{h_{n}}\right\}_{n \in \mathbb{N}} \subset \mathcal{K}_{\varepsilon, h_{n}}\left(\mathcal{S}_{h_{n}}, \Omega\right)$ such that for $n \rightarrow \infty$ assumptions $(L 0)_{\mathcal{A}_{h}}$ are satisfied.

Proof. Let $\mathcal{S}_{\varepsilon, h_{n}, \text { plate }} \rightarrow \mathcal{S}_{\varepsilon, \text { plate }}$ strongly if $H^{2}(\Omega)$ and $v_{h_{n}} \rightarrow v$ weakly in $V(\Omega)$

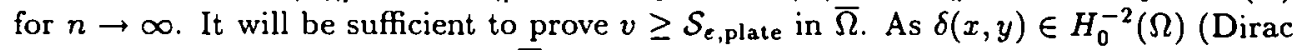
function, concentrated at $[x, y] \in \bar{\Omega})$ is linear continuous functional on $V(\Omega)$, we have $v_{h_{n}}(x, y) \rightarrow v(x, y)$ for all $[x, y] \in \bar{\Omega}$. Let us suppose that there exists $\left[x_{*}, y_{*}\right] \in \bar{\Omega}$ such that

$$
v\left(x_{*}, y_{*}\right)<\mathcal{S}_{\varepsilon, \text { plate }}\left(x_{*}, y_{*}\right)
$$


Moreover, since the element $v$ and $\mathcal{S}$ belong to the space $C(\bar{\Omega})$, estimate (4.40) holds in some neighbourhood $\mathcal{U}\left(\left[x_{*}, y_{*}\right], \varepsilon\right) \cap \bar{\Omega}(\varepsilon>0)$ where $\mathcal{U}\left(\left[x_{*}, y_{*}\right], \varepsilon\right)=\left\{[x, y] \in E_{2}\right.$ : $\left.\rho\left(\{x, y],\left[x_{*}, y_{*}\right]\right) \leq \varepsilon\right\}$. Further, $\operatorname{diam} G_{j} \leq h$ for any $G_{j} \in \mathcal{T}_{h}$ and $h \rightarrow 0_{+}$. This means there exists $A_{i, h_{0}} \in \mathcal{W}_{h_{0}}$ such that $A_{i, h_{0}} \in \mathcal{U}\left(\left[x_{*}, y_{*}\right], \varepsilon\right) \cap \bar{\Omega}$. Then by virtue of assumption (4.25) one has $A_{i, h_{0}} \in \mathcal{W}_{h}$ for any $h \leq h_{0}$. On the other hand, as $v_{h}\left(A_{i, h_{0}}\right) \geq \mathcal{S}_{\varepsilon, h, \text { plate }}\left(A_{i, h_{0}}\right)$ for any $h \leq h_{0}$, it must be $v\left(A_{i, h_{0}}\right)=\lim _{h \rightarrow 0_{+}} v_{h}\left(A_{i, h_{0}}\right) \geq$ $\mathcal{S}_{\varepsilon, \text { plate }}\left(A_{i, h_{0}}\right)$, which is a contradiction with previous considerations.

Consider an element $v \in \mathcal{K}(\mathcal{S}, \Omega)$. Then due to Lemma 6 a sequence $\left\{v_{o_{n}}\right\}_{n \in \mathbb{N}} \in$ $\Lambda_{\mathcal{K}(S, \Omega)}$ exist such that $\lim _{n \rightarrow \infty}\left\|v_{\mathbf{o}_{n}}-v\right\|_{H^{2}(\Omega)}=0$. Let $v_{h} \in V_{h}(\Omega)$ be such element, the restriction of which in $G_{j} \in \mathcal{T}_{h}$ is the Hermite bicubic interpolates of $v$. Then by definition one has $\left.v_{h_{n}}\right|_{G_{j}}=\mathcal{R}_{e_{h_{n}} e G_{G_{j}}} v$ and $\mathcal{R}_{e_{h_{n}} e G_{j}} v \in Q_{3}\left(G_{j}\right)$ is determined from the conditions

$$
\left.\begin{array}{r}
\mathcal{R}_{e_{h_{n}} \text { el } \sigma_{j}} v\left(A_{i}\right)=v\left(A_{i}\right), \quad \frac{\partial\left(\mathcal{R}_{e_{h_{n}} e \mid \sigma_{j}} v\left(A_{i}\right)\right)}{\partial x}=\frac{\partial v\left(A_{i}\right)}{\partial x} \\
\frac{\partial\left(\mathcal{R}_{e_{h_{n}} e \mid \sigma_{j}} v\left(A_{i}\right)\right)}{\partial y}=\frac{\partial v\left(A_{i}\right)}{\partial y}, \quad \frac{\partial^{2}\left(\mathcal{R}_{e_{h_{n}} e \mid \sigma_{j}} v\left(A_{i}\right)\right)}{\partial x \partial y}=\frac{\partial^{2} v\left(A_{i}\right)}{\partial x \partial y}
\end{array}\right\}
$$

where $\left[A_{i}\right]_{i=1}^{4}$ are vertices of $G_{j}$.

Denote by $o_{h_{n}}=\mathcal{R}_{e_{h_{n}} e} v_{o_{n}}$ the $V_{h}(\Omega)$-interpolate of $v_{o_{n}}$ over the partition $\mathcal{T}_{h}$. Then $o_{h_{n}} \in \mathcal{K}_{h_{n}}\left(\mathcal{S}_{h_{n}}, \Omega\right)$ holds, since the nodal parameters involve all values $v_{o_{n}}\left(A_{i}\right)$. Furthermore,

$$
\left\|\mathcal{R}_{e_{h_{n}} e} v_{o_{n}}-v_{o_{n}}\right\|_{H^{2}(\Omega)} \leq M h_{n}^{2}\left\|v_{o_{n}}\right\|_{H^{4}(\Omega)} . \quad\left(=O\left(h_{n}^{2}\right) \text { for } h_{n} \rightarrow 0_{+}\right)
$$

holds for any regular family $\left\{\mathcal{T}_{h}\right\}$ and therefore $\lim _{h_{n} \rightarrow 0_{+}}\left\|o_{h_{n}}-v\right\|_{H^{2}(\Omega)}=0$. Consequently, condition $(L 0)_{\mathcal{A}_{h}}$ is verified

Moreover, it remains to verify condition $(L 0)_{B_{h}}$.

Lemma 11. For every fixed $v \in \Lambda_{\mathrm{o}_{\mathrm{a}}(S, \Omega)}=\mathcal{O}_{e}(S, \Omega) \cap C_{0}^{\infty}(\Omega)$ there exists a sequence $\left\{v_{h_{n}}\right\}_{n \in \mathbb{N}} \subset \mathcal{O}_{e, h_{n}}\left(\mathcal{S}_{h_{n}}, \Omega\right)$ such that for $n \rightarrow \infty$ assumptions $(L 0)_{B_{n}}$ are satisfied.

Proof. We consider any $\mathcal{Q} \in C^{\infty}(\bar{\Omega})$ with $\mathcal{Q} \geq 0$ and define $\mathcal{Q}_{h}=\sum_{G \subset \Omega} \mathcal{Q}\left(o_{G}\right) \mathcal{X}_{G}$, where $\mathcal{X}_{G}$ is the characteristic function of the set $G$ and $o_{G}$ is the centroid of $G$. Then we have

$$
\lim _{h_{n} \rightarrow 0_{+}} \int_{\Omega}\left(v_{h_{n}}-\mathcal{S}_{e, h_{n}, \text { mem }}\right) \mathcal{Q}_{h_{n}} d \Omega=\int_{\Omega}\left(v-\mathcal{S}_{e, \text { mem }}\right) \mathcal{Q} d \Omega
$$

since $v_{h_{n}} \rightarrow v$ weakly in $H_{0}^{1}(\Omega)$ and $\mathcal{Q}_{h_{n}} \rightarrow \mathcal{Q}, \mathcal{S}_{\varepsilon, h_{n}, \text { mem }} \rightarrow \mathcal{S}_{\varepsilon, \text { mem }}$ strongly in $L_{2}(\Omega)$ and taking into account the Rellich theorem. On the other hand, one has

$$
\int_{\Omega}\left(v_{h_{n}}-\mathcal{S}_{e, h_{n}, \text { mem }}\right) \mathcal{Q}_{h_{n}} d \Omega=\sum_{G, \bar{\Omega}} \mathcal{Q}\left(o_{G}\right) \int_{G}\left(v_{h_{n}}-\mathcal{S}_{\varepsilon, h_{n}, \text { mem }}\right) d \Omega .
$$

Then due to Simpson's integral formula and the definition of $\mathcal{O}_{\varepsilon, h}\left(\mathcal{S}_{h}, \Omega\right)$, we obtain

$$
\int_{\Omega}\left(v_{h_{n}}-\mathcal{S}_{\ell, h_{n}, \text { mem }}\right) d \Omega=\frac{1}{4}(\text { meas } G) \sum_{i=1}^{4}\left(v_{h_{n}}-\mathcal{S}_{\varepsilon, h_{n}, \text { mem }}\right)\left(A_{i}\right) \geq 0
$$


where $A_{i} \in \dot{\mathcal{W}}_{h}$. Thus by virtue of (4.42) and (4.43), we arrive at

$$
\int_{\Omega}\left(v_{h_{n}}-\mathcal{S}_{\varepsilon, h_{n}, \text { mem }}\right) \mathcal{Q}_{h_{n}} d \dot{\Omega} \geq 0
$$

Then (4.41) implies $\int_{\Omega}\left(v-\mathcal{S}_{\varepsilon, \text { mem }}\right) \mathcal{Q} d \Omega \geq 0$ which in turn implies $v \geq \mathcal{S}+\varepsilon H_{\text {mem }}$ a.e. in $\Omega$, i.e. $v \in \mathcal{O}_{\varepsilon}(\mathcal{S}, \Omega)$.

Let $v \in \Lambda_{o_{\varepsilon}(S, \Omega)}$. There exists a $\mathcal{S}_{\varepsilon, \text { mem }} \in H^{2}(\Omega)$ such that $\mathcal{S}_{\varepsilon, \text { mem }}=0$ on $\partial \Omega$. Then we have

$$
v-\mathcal{S}_{\epsilon, \text { mem }}=\theta \in \mathcal{O}_{*}(\Omega)=\left\{w \in H_{0}^{1}(\Omega): w \geq 0 \text { a.e. in } \Omega\right\} .
$$

Let us employ a regularization operator $R_{\mathcal{K}}$ with the kernel $A \exp [((x, y)-(\xi, \eta)) / \mathcal{K}]$, $A$ a constant. Let $R_{\mathcal{K}} E S_{\varepsilon, \text { mem }}$ and $R_{\mathcal{K}} E \theta$ denote the regularization applied to a proper extension of the functions $\mathcal{S}_{\varepsilon, \text { mem }}$ and $\theta$ to a larger domain $\Omega_{*} \supset \bar{\Omega}$, so that $R_{\mathcal{K}} E \theta \geq 0$. We define

$$
\begin{aligned}
v_{h_{n}}= & \mathcal{E}_{h_{n}}\left(R_{\mathcal{K}} E \mathcal{S}_{e, \text { mem }} \mathcal{R}_{\mathcal{K}} E \theta\right. \\
& \left.+\left(\left\|R_{\mathcal{K}} E \mathcal{S}_{\varepsilon, \text { mem }}-\mathcal{S}_{e, \text { mem }}\right\|_{C(\bar{\Omega})}+\left\|\mathcal{S}_{e, \text { mem }}-\mathcal{S}_{e, h_{n}, \text { mem }}\right\|_{C(\bar{\Omega})}\right) \vartheta\right)
\end{aligned}
$$

where $\mathcal{E}_{h_{n}}: C(\bar{\Omega}) \rightarrow W_{h}(\Omega)$ denotes the Lagrange quadratic interpolation and $\vartheta \in$ $C_{0}^{\infty}\left(B \rho\left(x_{0}, y_{0}\right)\right)$ a non-negative function with $\left[x_{0}, y_{0}\right] \in S(w):=\{[w, y] \in \Omega: w(x, y)>$ $\left.\mathcal{S}_{\varepsilon, \text { mem }}(x, y)\right\}$ and $B_{p}\left(x_{0}, y_{0}\right) \subset S(w)$ a ball. Consequently, $v_{h_{n}} \in W_{h_{n}}(\Omega)$, and for any node $A \in \dot{\mathcal{W}}_{h}$ we have

$$
\begin{aligned}
v_{h_{n}}(A) \geq & R_{\mathcal{K}} E \mathcal{S}_{\varepsilon, \mathrm{mem}}(A) \\
& +\left|\mathcal{S}_{\varepsilon, \mathrm{mem}}(A)-R_{\mathcal{K}} E \mathcal{S}_{\epsilon, \mathrm{mem}}(A)\right|+\left|\mathcal{S}_{\ell, h, \mathrm{mem}}(A)-\mathcal{S}_{\epsilon, \mathrm{mem}}(A)\right| \\
\geq & \mathcal{S}_{\varepsilon, h, \text { mem }}(A)
\end{aligned}
$$

so that $v_{h_{n}} \in \mathcal{O}_{\varepsilon, h_{n}}\left(\mathcal{S}_{h_{n}}, \Omega\right)$. Furthermore, we may write

$$
\begin{aligned}
\mid v_{h_{n}} & -\left.v\right|_{H^{1}(\Omega)} \\
= & \mid \mathcal{E}_{h_{n}}\left(R_{\mathcal{K}} E \mathcal{S}_{\varepsilon, \mathrm{mem}}\right)-\mathcal{S}_{\varepsilon, \mathrm{mem}}+\mathcal{E}_{h_{n}}\left(R_{\mathcal{K}} E \theta\right)-\theta \\
& \times\left.\left(\left\|R_{\mathcal{K}} E \mathcal{S}_{\varepsilon, \mathrm{mem}}-\mathcal{S}_{\epsilon, \mathrm{mem}}\right\|_{C(\bar{\Omega})}+\left\|\mathcal{S}_{\varepsilon, \mathrm{mem}}-\mathcal{S}_{\varepsilon, h_{n}, \mathrm{mem}}\right\|_{C(\bar{\Omega})}\right) \mathcal{E}_{h_{n}} \vartheta\right|_{H^{1}(\Omega)} \\
\leq & \left|\mathcal{E}_{h_{n}}\left(R_{\mathcal{K}} E \mathcal{S}_{\varepsilon, \mathrm{mem}}\right)-R_{\mathcal{K}} E \mathcal{S}_{\varepsilon, \mathrm{mem}}\right|_{H^{1}(\Omega)}+\left|R_{\mathcal{K}} E \theta-\theta\right|_{H^{1}(\Omega)} \\
& +\left|\mathcal{E}_{h_{n}}\left(R_{\mathcal{K}} E \theta\right)-R_{\mathcal{K}} E \theta\right|_{H^{1}(\Omega)}+\left|R_{\mathcal{K}} E \mathcal{S}_{\varepsilon, \mathrm{mem}}-\mathcal{S}_{\varepsilon, \mathrm{mem}}\right|_{H^{1}(\Omega)} \\
& +\left(\left\|R_{\mathcal{K}} E \mathcal{S}_{\varepsilon, \mathrm{mem}}-\mathcal{S}_{\varepsilon, \mathrm{mem}}\right\|_{C(\bar{\Omega})}\right. \\
& \left.+\left\|\mathcal{S}_{\varepsilon, \mathrm{mem}}-\mathcal{S}_{\varepsilon, h_{n}, \mathrm{mem}}\right\|_{C(\bar{\Omega})}\right)\left.\mathcal{E}_{h_{n}} \vartheta\right|_{H^{1}(\Omega)} \\
\rightarrow & 0
\end{aligned}
$$

as $\mathcal{K} \rightarrow 0_{+}$and $h_{n} \rightarrow 0_{+}$. Here we have used the facts that $\mathcal{S}_{\varepsilon, \text { mem }} \in H^{2}(\Omega)$ and

$$
\left\|R_{\mathcal{K}} E \mathcal{S}_{\ell, \text { mem }}-\mathcal{S}_{\ell, \mathrm{mem}}\right\|_{C(\bar{\Omega})} \leq \mathrm{const}\left\|R_{\mathcal{K}} E \mathcal{S}_{\ell, \mathrm{mem}}-\mathcal{S}_{\varepsilon, \mathrm{mem}}\right\|_{H^{2}(\Omega)} \rightarrow 0
$$

as $\mathcal{K} \rightarrow 0_{+}$(the seminorm in $H^{1}(\Omega)$ be denoted by $\left.|\cdot|_{H^{1}(\Omega)}\right)$. This means that the assumptions of $(L 0)_{B_{h}}$ are fulfilled 
Let $u_{0}\left(e_{0}\right) \in W(\Omega)$ be the solution of (1.23) and $u_{0 h_{n}}\left(e_{0 h_{n}}^{*}\right) \in W_{h_{n}}(\Omega)$ that of (2.2). Taking into account (4.31) and Lemma 9 a standard estimate gives

$$
\left\|u_{0}\left(e_{0}^{*}\right)-u_{0 h_{n}}\left(c_{0 h_{n}}\right)\right\|_{H_{0}^{1}(\Omega)} \leq M h_{n}\left\|u_{0}\left(e_{0}^{*}\right)\right\|_{H^{2}(\Omega)} \leq M_{*} h_{n} .
$$

Therefore, as $u_{0}\left(e_{0}\right) \in \mathcal{O}_{\varepsilon}(\mathcal{S}, \Omega), u_{0 h_{n}}\left(e_{0 h_{n}}\right) \in \mathcal{O}_{\varepsilon, h_{n}}\left(\mathcal{S}_{h_{n}}, \Omega\right)$ for $h_{n}$ sufficiently small.

4.2 Transition from a plate to a membrane. From the above mentioned arguments and due to Lemmas $4-8$ all assumptions of Theorems $1-3$ are satisfied. This means that there exists at least one solution $e_{\varepsilon}^{*}=\left[E_{\varepsilon}^{*}, q_{\varepsilon}^{*}, \mathcal{S}_{\varepsilon}^{*}\right] \in U_{a d}(\Omega)$, the solution for $\left(\mathcal{P}_{\varepsilon}\right)$ for every $\left(\hat{\alpha}_{B} / 2\right) \geq \varepsilon>h$ and $e_{0}^{*}=\left[E_{0}^{*}, q_{0}^{*}, \mathcal{S}_{0}^{*}\right] \in U_{a d}(\Omega)$ the solution for problem $\left(\mathcal{P}_{0}\right)$ for the cost functional:

$$
\mathcal{L}(e, u(e))=\int_{\Omega}\left[u_{\varepsilon}(e)-z_{d}\right]^{2} d \Omega
$$

Particularly, there is a sequence $\left\{\varepsilon_{n_{k}}\right\}_{k \in \mathbb{N}}, \varepsilon_{n_{k}} \rightarrow 0$, for which

$$
\left.\begin{array}{rl}
E_{\varepsilon_{n_{k}}}^{*} & \rightarrow E_{0}^{*} \text { in } R \\
q_{\varepsilon_{n_{k}}}^{*} & \rightarrow q_{0}^{*} \text { strongly in } C(\bar{\Omega}) \\
\mathcal{S}_{\varepsilon_{n_{k}}}^{*} & \rightarrow \mathcal{S}_{0}^{*} \text { strongly in } H^{2}(\Omega) \\
u_{\varepsilon_{n_{k}}}\left(e_{\varepsilon_{n_{k}}^{*}}^{*}\right) & \rightarrow u_{0}\left(e_{0}^{*}\right) \text { weakly in } W(\Omega) .
\end{array}\right\}
$$

If the limit state function $u_{0}\left(e_{0}^{*}\right)$ is such that $u_{0}\left(e_{0}^{*}\right) \in \mathcal{K}_{\varepsilon}\left(\mathcal{S}_{0}^{*}, \Omega\right)$ and $\frac{1}{\varepsilon_{n_{k}}} \| \mathcal{J}\left(q_{e_{n_{k}}^{*}}^{*}\right)-$ $\mathcal{J}\left(q_{0}^{*}\right) \|_{L_{2}(\Omega)} \rightarrow 0$ for $\varepsilon_{n_{k}} \rightarrow 0$, then one has

$$
\left\|u_{\varepsilon_{n_{k}}}\left(e_{\varepsilon_{n_{k}}^{*}}^{*}\right)-u_{0}\left(e_{0}^{*}\right)\right\|_{W(\Omega)}=\dot{O}\left(\sqrt{\varepsilon_{n_{k}}}\right) \quad \text { for } \varepsilon_{n_{k}} \rightarrow 0
$$

where $\|v\|_{W(\Omega)}:=\|v\|_{H_{0}^{1}(\Omega)}$ for $v \in W(\Omega)$ and

$$
u_{\varepsilon_{n_{k}}}\left(e_{\varepsilon_{n_{k}}^{*}}^{*}\right) \rightarrow u_{0}\left(e_{0}^{*}\right) \quad \text { strongly in } V(\Omega)
$$

where $\|v\|_{V(\Omega)}:=\|v\|_{H^{2}(\Omega)}$ for $v \in V(\Omega)$. Indeed, taking $v=u_{0}\left(e_{0}^{*}\right)$ in the variational inequality

$$
\begin{gathered}
\left\langle\varepsilon_{n_{k}} \mathcal{A}\left(E_{\varepsilon_{n_{k}}}^{*}\right) u_{\varepsilon_{n_{k}}}\left(e_{\varepsilon_{n_{k}}^{*}}^{*}\right)+\mathcal{B}\left(E_{\varepsilon_{n_{k}}}^{*}\right) u_{\varepsilon_{n_{k}}}\left(e_{\varepsilon_{n_{k}}}^{*}\right), v-u_{e_{n_{k}}}\left(e_{\varepsilon_{n_{k}}}^{*}\right)\right\rangle_{V(\Omega)} \\
\geq\left\langle\mathcal{J}\left(q_{\varepsilon_{n_{k}}^{*}}^{*}\right), v-u_{\varepsilon_{n_{k}}}\left(e_{\varepsilon_{n_{k}}^{*}}^{*}\right)\right\rangle_{W(\Omega)}
\end{gathered}
$$

$v \in \mathcal{K}_{\varepsilon}\left(\mathcal{S}_{n_{k}}^{*}, \Omega\right)$, and $v=u_{c_{n_{k}}}\left(e_{\varepsilon_{n_{k}}}^{*}\right)$ in the variational inequality

$$
\left\langle\mathcal{B}\left(E_{0}^{*}\right) u_{0}\left(e_{0}^{*}\right), v-u_{0}\left(e_{0}^{*}\right)\right\rangle_{W(\Omega)} \geq\left\langle\mathcal{J}\left(q_{0}^{*}\right), v-u_{0}\left(e_{0}^{*}\right)\right\rangle_{W(\Omega)} \quad\left(v \in \mathcal{O}_{e}\left(\mathcal{S}_{0}^{*}, \Omega\right)\right)
$$

we obtain

$$
\begin{aligned}
& \alpha_{\mathcal{A}}\left\|u_{\varepsilon_{n_{k}}}\left(e_{\varepsilon_{n_{k}}^{*}}^{*}\right)-u_{0}\left(e_{0}^{*}\right)\right\|_{V(\Omega)}^{2}+\frac{\alpha_{B}}{\varepsilon_{n_{k}}}\left\|u_{\varepsilon_{n_{k}}}\left(e_{\varepsilon_{n_{k}}^{*}}^{*}\right)-u_{0}\left(e_{0}^{*}\right)\right\|_{W(\Omega)}^{2} \\
& \leq\left\langle\mathcal{A}\left(E_{0}^{*}\right) u_{0}\left(e_{0}^{*}\right), u_{0}\left(e_{0}^{*}\right)-u_{\varepsilon_{n_{k}}}\left(e_{e_{n_{k}}^{*}}\right)\right\rangle_{V(\Omega)} \\
&+\frac{1}{\varepsilon_{n_{k}}}\left\|\mathcal{J}\left(q_{e_{n_{k}}^{*}}^{*}\right)-\mathcal{J}\left(q_{0}^{*}\right)\right\|_{L_{2}(\Omega)}\left\|u_{\varepsilon_{n_{k}}}\left(e_{\varepsilon_{n_{k}}^{*}}^{*}\right)-u_{0}\left(e_{0}^{*}\right)\right\|_{W(\Omega)}
\end{aligned}
$$


which yields the first estimate

$$
\begin{aligned}
& \left\|u_{\varepsilon_{n_{k}}}\left(e_{\varepsilon_{n_{k}}}^{*}\right)-u_{0}\left(e_{0}^{*}\right)\right\|_{V(\Omega)} \\
& \quad \leq \frac{1}{\alpha_{\mathcal{A}}}\left(\left\|\mathcal{A}\left(E_{0}^{*}\right) u_{0}\left(e_{0}^{*}\right)\right\|_{V^{*}(\Omega)}+M\left(\frac{1}{\varepsilon_{n_{k}}}\right)\left\|\mathcal{J}\left(q_{\varepsilon_{n_{k}}}^{*}\right)-\mathcal{J}\left(q_{0}^{*}\right)\right\|_{L_{2}(\Omega)}\right)
\end{aligned}
$$

and afterwards the estimate

$$
\begin{aligned}
& \left\|u_{\varepsilon_{n_{k}}}\left(e_{e_{n_{k}}^{*}}^{*}\right)-u_{0}\left(e_{0}^{*}\right)\right\|_{W(\Omega)} \\
& \quad \leq \frac{\varepsilon_{n_{k}}}{\alpha_{\mathcal{B}}}\left(\frac{1}{M}\left\|\mathcal{A}\left(E_{0}^{*}\right) u_{0}\left(e_{0}^{*}\right)\right\|_{V \cdot(\Omega)}+\frac{1}{\varepsilon_{n_{k}}}\left\|\mathcal{J}\left(q_{e_{n_{k}}^{*}}^{*}\right)-\mathcal{J}\left(q_{0}^{*}\right)\right\|_{L_{2}(\Omega)}\right) .
\end{aligned}
$$

Hence (4.45) holds and one has $u_{e_{n_{k}}}\left(e_{e_{n_{k}}^{*}}\right) \rightarrow u_{0}\left(e_{0}^{*}\right)$ weakly in $V(\Omega)$, by virtue of $(4.48)$ and (4.49). Then using this fact in (4.47), one concludes the strong convergence from the inequality

$$
\begin{aligned}
\limsup _{\varepsilon_{n_{k}} \rightarrow 0} & \alpha_{\mathcal{A}}\left\|u_{e_{n_{k}}}\left(e_{\varepsilon_{n_{k}}^{*}}^{*}\right)-u_{0}\left(e_{0}^{*}\right)\right\|_{V(\Omega)}^{2} \\
\leq & \lim _{e_{n_{k}} \rightarrow 0}\left\langle\mathcal{A}\left(E_{0}^{*}\right) u_{0}\left(e_{0}^{*}\right), u_{\varepsilon_{n_{k}}}\left(e_{\varepsilon_{n_{k}}^{*}}^{*}\right)-u_{0}\left(e_{0}^{*}\right)\right\rangle_{V(\Omega)} \\
& +\lim _{\varepsilon_{n_{k}} \rightarrow 0} \frac{1}{\varepsilon_{n_{k}}}\left\|\mathcal{J}\left(q_{\varepsilon_{n_{k}}}^{*}\right)-\mathcal{J}\left(q_{0}^{*}\right)\right\|_{L_{2}(\Omega)}\left\|u_{e_{n_{k}}}\left(e_{e_{n_{k}}^{*}}^{*}\right)-u_{0}\left(e_{0}^{*}\right)\right\|_{W(\Omega)} \\
= & 0 .
\end{aligned}
$$

Remark. One can also consider the case of the partially clamped plate by considering the non-empty, closed convex subset

$$
\mathcal{K}_{\varepsilon, \operatorname{clam}}(\mathcal{S}, \Omega)=\left\{v \in V(\Omega): \operatorname{tr}(v) \geq 0 \text { on } \partial \Omega_{c}\right\}
$$

of

$$
V(\Omega)=\left\{v \in H^{2}(\Omega): \operatorname{tr}(v)=0 \text { and } \frac{\partial \operatorname{tr}(v)}{\partial n}=0 \text { on } \partial \Omega_{u}\right\}
$$

instead of $\mathcal{K}_{\varepsilon}(\mathcal{S}, \Omega)$ defined by $\left(4.1_{a}\right)$. Clearly, $\emptyset \neq \mathcal{K}_{\varepsilon, \text { clam }}(\mathcal{S}, \Omega) \subset \mathcal{K}_{\varepsilon}(\mathcal{S}, \Omega)$. Under the definition of $U_{a d}(\Omega)$, if $u_{\varepsilon}^{*}(e)$ denotes the unique solution to the obstacle problem for the partially clamped plate, one can apply Theorem 3 in order to conclude $u_{\varepsilon}^{*}(e) \rightarrow u(e)$ strongly in $W(\Omega) \quad\left(=\left\{v \in H^{1}(\Omega): \operatorname{tr}(v)=0\right.\right.$ on $\left.\left.\partial \Omega_{u}\right\}\right)$ (note that one still has $\operatorname{cl} \mathcal{K}_{\varepsilon, \text { clam }}(\mathcal{S}, \Omega)=\mathcal{O}_{\varepsilon}(\mathcal{S}, \Omega)$ with closure taken in $\left.W(\Omega)\right)$, where $\mathcal{O}_{\varepsilon}(\mathcal{S}, \Omega)$ is defined by $\left(4.1_{b}\right)$ and $u(e)$ is the corresponding solution for the membrane. Since, however, in general $u(e) \notin V(\Omega)$ one cannot expect to improve that we have convergence in $H^{2}(\Omega)$. In this case a boundary layer arises $[15,16]$.

Finally, by virtue of Theorem 4 (under the above conditions and Lemmas 9 - 11), for any regular family of partitions $\left\{\mathcal{T}_{h}\right\}$, refining $\mathcal{T}_{h_{0}}$, relations $(2.12)$ and (2.14) for the membrane approximation to the plate with inner obstacle hold.

Acknowledgement. The author is deeply grateful to Prof. Panagiotopoulos for many suggestions and corrections which let to an essential improvement of the paper. 


\section{References}

[1] Adams, R. A.: Sobolev Spaces. New York - San Francisco - London: Acad. Press 1975.

[2] Barbu, V.: Optimal Control of Variational Inequalities (Pitman Advanced Publishing Program). Boston - London: Pitman 1987.

[3] Bock, I. and J. Lovišsek: An optimal control problems for variational inequalities with controls in coefficients and in unilateral constraints. Appl. Math. 32 (1987), $301-314$.

[4] Brezis, H. and G. Stampacchia: Remarks on some fourth-order variational inequalities. Ann. Scu. Norm. Sup. Pisa 4 (1977), 363 - 371.

[5] Duvaut, G. and J. L. Lions: Inequalities in Mechanics and Physics. Berlin: SpringerVerlag 1975.

[6] Eckhaus, W. and J. K. Moet: Asymptotic solution in free boundary problems of singularly perturbed elliptic variational inequalities. In: Differential Equations and Applications (eds.: W. Eckhaus and E. M. Jaeger). Amsterdam: Noth-Holland Publ. Comp. 1978.

[7] Frank, L. S.: Singular Perturbations. Vol. I: Spaces and Singular Perturbations on Manifolds without Boundary. Amsterdam: North-Holland Publ. Comp. 1990.

[8] Greenlee, W. M.: Singular perturbations of eigenvalues. Arch. Rat. Mech. Anal. 34 (1969), $143-164$.

[9] Glowinski, R.: Numerical Methods for Nonlinear Variational Problems. New York: Springer-Verlag 1984.

[10] Huet, D.: Singular perturbations of elliptic problems. Annal. Math. Pura Appl. 95 (1973), 77 - 114 .

[11] Haslinger, J. P. and Neittaanmäki: Finite Elemenl Approximation for Optimal Shape Design. Theory and application. New York: J. Wiley 1988.

[12] Johnson, C.: Numerical Solution of Partial Differential Equations by the Finite Element Method. Cambridge: Univ. Press 1987.

[13] Kinderlehrer, D. and G. Stampacchia: An Introduction to Variational Inequalities and their Application. New York: Academic Press 1980.

[14] Khludnev, A. M. and J. Sokolowski: Modelling and Control in Solid Mechanics. Basel Boston - Berlin: Birkhäuser Verlag 1997.

[15] Lions, J. L.: Perturbations singuliéres dans les problémes aux limites et en contrôle optimal. Lect. Notes Math. 323 (1973).

[16] Lions, J. L.: Singular perturbations and singular layers in variational inequalities. In: Contribution to Nonlinear Functional Analysis (ed.: E. H. Zarantonello) New York: Academic Press 1971, pp. 523 - 564.

[17] Lovišek, J.: Optimal control of variational inequality with applications to axisymmetric shells. Appl. Math. Optim. 24 (1991), 1 - 33.

[18] Mignot, R. and J. P. Puel: Optimal control in some variational inequalities. SIAM J. Control Optim. 22 (1984), $466-476$.

[19] Mosco, U.: An introduction to the approximate solutions of variational inequalities. In: Constructive Aspects of Functional Analysis, Vol. II (ed.: G. Geymonat). Centro Internaz. Matem. 1971, pp. $497-682$.

[20] Panagiotopoulos, P. D.: Optimal control in unilateral thin plate theory. Arch. Mech. 29 (1977), $25-39$.

[21] Rodrigues, J. F.: Obstacle Problems in Mathematical Physics (North-Holland Mathematical Studies: Vol. 134). Amsterdam: North-Holland 1989. 
[22] Sanchez-Palencia, E.: Non-Homogeneous Media and Vibration Theory. Berlin: SpringerVerlag 1980.

[23] Reismann, H.: Elastic plates. Theory and Application. New York: John Wiley \& Sons 1988.

[24] Roos, H.-G., Stynes, M. and L. Tobiska: Numerical Methods for Singularly Perturbed Differential Equations. Convention-Diffusion and Flow Problems. Berlin - Heidelberg: Springer-Verlag 1996.

Received 28.04.1995; in revised form 10.06.1999 